\title{
Álgebras estandarmente estratificadas e álgebras quase-hereditárias
}

\section{Paula Andrea Cadavid Salazar}

\author{
DISSERTAÇÃO APRESENTADA \\ $\mathrm{AO}$ \\ INSTITUTO DE MATEMÁTICA E ESTATÍSTICA \\ DA \\ UNIVERSIDADE DE SÃO PAULO \\ PARA \\ OBTENÇÃA DO TÍTULO \\ $\mathrm{DE}$ \\ MESTRE EM CIÊNCIAS
}

Área de Concentração: Matemática

Orientador: Prof. Dr. Eduardo do Nascimento Marcos

Durante o desenvolvimento deste trabalho o autor recebeu auxílio financeiro da $\mathrm{CNPq}$

São Paulo, outubro de 2007 


\section{Álgebras estandarmente estratificadas e álgebras quase-hereditárias}

Este exemplar corresponde à dissertação defendida por Paula Andrea Cadavid Salazar

e aprovada pela comissão julgadora.

São Paulo, 15 de fevereiro de 2008.

Banca examinadora:

Prof. Dr. Eduardo do Nascimento Marcos (IME-USP)

Prof. Dr. Maria Izabel Ramalho Martins (IME-USP)

Prof. Dr. Marcelo Lanzilotta Mernies (Univ. de la Rep., Uruguay) 
A Luisa Fernanda 


\section{Agradecimentos}

Ao IME por ter-me concedido ótimas condições de trabalho, em especial ao professor Eduardo do Nascimento Marcos pela sua orientação e à professora María Izabel Ramalho Martins pela sua valiosa ajuda.

A todos os amigos do IME, especialmente a Natalia, Mary Luz e ao Pablo, por estar sempre nos momentos difícies.

Ao CNPq, pelo apoio financeiro. 


\section{Resumo}

Sejam $K$ um corpo algebricamente fechado, $A$ uma $K$-álgebra básica conexa de dimensão finita sobre $K$ e $\boldsymbol{e}=\left(e_{1}, e_{2}, \ldots, e_{n}\right)$ um conjunto completo de idempotentes ortogonais, primitivos e ordenados de $A$. O conjunto dos módulos estandares é o conjunto $\triangle=\left\{\triangle_{1}, \ldots, \triangle_{n}\right\}$, onde $\triangle_{i}$ é o quociente maximal do $A$-módulo projetivo $P_{i}$ com fatores de composição simples $S_{j}$, com $j \leq i, \mathcal{F}(\triangle)$ é a subcategoria plena de $\bmod A$ dos módulos têm uma $\triangle$-filtração. Se $A_{A} \in \mathcal{F}(\triangle)$ diz-se que $A$ é uma álgebra estandarmente estratificada. Se, além disso, para cada elemento em $\triangle$ vale que $\operatorname{End}_{A}\left(\triangle_{i}\right) \cong K$ diz-se que $A$ é uma álgebra álgebra quase-hereditária. Nesta disertação estudamos as propriedades de $\mathcal{F}(\triangle)$, especialmente quando $A$ é estandarmente estratificada, e algumas condições necessárias e suficientes para que $A$ seja quase-hereditária.

Palavras-chave: Álgebras estandarmente estratificadas, álgebras quasehereditárias, módulos estandares. 


\section{Abstract}

Let $K$ be an algebraically closed field, $A$ a basic, connected, finite dimensional $K$-algebra and $\boldsymbol{e}=\left(e_{1}, e_{2}, \ldots, e_{n}\right)$ a complete set of ordered primitive orthogonal idempotents of $A$. The set of standard modules is the set $\triangle=\left\{\triangle_{1}, \ldots, \triangle_{n}\right\}$, where $\triangle_{i}$ is the maximal factor submodule of $P_{i}$ whose composition factors are isomorphic to $S_{j}$, for $j \leq i$. We denote by $\mathcal{F}(\triangle)$ the full subcategory of $\bmod A$ containing the modules which are filtered by modules in $\triangle$. If $A_{A} \in \mathcal{F}(\triangle)$ we say that $A$ is standardly stratified. Moreover, if $\operatorname{End}_{A}\left(\triangle_{i}\right) \cong K$ for each element in $\triangle$ we say that $A$ is quasi hereditary. In this work we study the properties of the category $\mathcal{F}(\triangle)$, especially when $A$ is stardardly stratified, and some necessary and sufficient conditions to $A$ be quasi hereditary.

Keywords: standardly stratified algebras, quasi-hereditary algebras, standard modules. 


\section{Sumário}

1 Um pouco de Teoria de Representações 1

1.1 Preliminares . . . . . . . . . . . . . . . . . . 1

1.2 Carcases e álgebras de caminho . . . . . . . . . . . . . 3

1.3 Representações e módulos . . . . . . . . . . . . . . . . . 6

1.4 Seqüências de Auslander-Reiten . . . . . . . . . . . . . . . . . 10

2 Seqüências de Auslander-Reiten relativas 14

3 Álgebras estandarmente estratificadas e álgebras quase he$\begin{array}{ll}\text { reditárias } & 29\end{array}$

3.1 Sobre o grupo de Grothendieck . . . . . . . . . . . . . 30

3.2 Módulos estandares e coestandares . . . . . . . . . . . . . 33

3.3 As categorias $\mathcal{F}(\triangle)$ e $\mathcal{F}(\nabla) \ldots \ldots . \ldots . \ldots 39$

3.4 Álgebras estandarmente estratificadas . . . . . . . . . . . . 47

3.5 Álgebras estandarmente estratificadas e módulos inclinantes . 60

3.6 Álgebras quase-hereditárias . . . . . . . . . . . . . . . 81

$\begin{array}{lr}\text { Referências Bibliográficas } & 87\end{array}$ 


\section{Introdução}

As álgebras quase-hereditárias foram introduzidas por E. Cline, B. Parshall e L. Scott, em [9] e [10], no contexto da teoria algébrica de grupos e na representação das álgebras de Lie complexas semisimples de dimensão finita, mais precisamente no estudo da categoria dos pesos máximos. V. Dlab e C. M. Ringel fizeram um extenso estudo desta classe de álgebras e de suas categorias de módulos. Um dos principais resultados obtidos é a existencia do módulo característico que foi dado por Ringel em [24]. Tal módulo, que é tanto inclinante como coinclinante, estabelece uma conexão com a teoria de inclinação que tem sido muito desenvolvida nos ultimos anos.

Mais tarde, E. Cline, B. Parshall e L. Scott introduziram em [11] um conceito mais geral: as álgebras estandarmente estratificadas.

As álgebras quase-hereditárias não são nada mais do que as álgebras estandarmente estratificadas de dimensão global finita. Elas aparecem, por exemplo, no estudo da categoria $\mathcal{O}$ de Berstein, Gelfand e Gelfand associada com a decomposição triangular de uma álgebra de Lie complexa, semisimples de dimensão finita.

Varias geralizações tem sido feitas, uma delas apareceu em [16], onde K. Erdman e C. Sáenz definem os sistemas estratificantes. Posteriormente E. Marcos, O. Mendoza e C. Sáenz em [22], usam tais sistemas para geralizar os resultados obtidos por I. Ágoston, D. Happel, E. Lukás e L. Unger em [1] acerca da dimensão finitística de uma álgebra estandarmente estratificada.

Os conceitos centrais nesta teoria são os conjuntos $\triangle$ e $\nabla$ dos módulos 
estandares e coestandares, respectivamente. Nosso principal objetivo nesta dissertação é estudar algumas propriedades das categorias $\mathcal{F}(\triangle)$ e $\mathcal{F}(\nabla)$, dos módulos filtrados por $\triangle$ e $\nabla$, respectivamente, fazendo ênfase no caso em que a álgebra é estandarmente estratificada. Para tal esta dissertação esta organizada como segue:

O Capítulo 1 destina-se a revisão de conceitos, definições, teoremas e notações que serão usadas ao longo deste texto, seu principal objetivo é tentar tornar a dissertação autocontida.

No Capítulo 2, baseados no trabalho de Ringel em [24], demonstramos que a categoria $\mathcal{F}(\theta)$, onde $\theta=\{\theta(1), \theta(2), \ldots, \theta(n)\}$ é um conjunto de módulos tal que $\operatorname{Ext}_{A}^{1}(\theta(i), \theta(j))=0$, para $i \leq j$, admite seqüências de AuslarderReiten relativas.

No Capítulo 3, na primeira seção definimos o grupo de Grothendieck e enunciamos algumas propriedades a usar durante o restante do capítulo. Na segunda seção demonstramos as propriedades básicas dos módulos estandares. Na seção 3.3 apresentamos propriedades homológicas das categorias $\mathcal{F}(\triangle)$ e $\mathcal{F}(\nabla)$, em particular que são funtorialmente finitas e que admitem seqüências de Auslanden-Relativas. Tais resultados foram extraídos de [13],[15], [26] e [24]. Na seção 3.4 introduzimos os conceitos de álgebra estandarmente estratificada e álgebra quase-hereditária, demonstramos que quando a álgebra é estandarmente estratificada os módulos em $\mathcal{F}(\triangle)$ têm dimensão projetiva finita e que as álgebras quase-hereditárias tem dimensão global finita. Os resultados desta seção estão contidos em [15]. Na seção 3.5 demonstramos que se $A$ é uma álgebra estandarmente estratificada existe um $A$-módulo inclinante generalizado $T$ associado a $A$, tal que $\mathcal{F}(\triangle) \cap \mathcal{Y}(\triangle)=$ 
$a d d T$ e que o anel de endomorfismos $B=\operatorname{End}_{A}\left(T_{A}\right)$ é de novo uma $K$-álgebra estandarmente estratificada. Mais ainda, que $\operatorname{End}_{B}\left(T^{\prime}\right)$ é Morita equivalente a $A$, onde $T^{\prime}$ é o $B$-módulo inclinante generalizado associado a $B$. Os resultados apresentados nesta seção estão contidos no trabalho de C. Xi, em [26]. Finalmente, na Seção 3.6 abordaremos duas caracterizações diferentes das álgebras quase-hereditárias. 


\section{Capítulo 1}

\section{Um pouco de Teoria de Representações}

Este capítulo traz uma série de conceitos, definições, teoremas e notações que utilizaremos livremente em todo o restante deste texto. Grande parte dos tópicos aqui apresentados são bastante familiares, mas ainda assim serão revisados para fixar notações e tornar a terminologia do trabalho mais uniforme.

Por outro lado vale alertar que não temos nenhuma pretensão de fornecer um tratamento completo dos assuntos aqui abordados. Detalhes de tais conceitos podem ser encontrados em vários livros, mas todos os aqui mencionados podem ser achados em [4].

\subsection{Preliminares}

Faremos aqui um percurso muito breve sobre algumas noções de álgebras associativas de dimensão finita que utilizaremos ao longo desta dissertação. 
Para consultar detalhes citamos o Capítulo I e o Apêndice A de [4].

Se $A$ é uma $K$-álgebra de dimensão finita, então o módulo $A_{A}$ admite uma decomposição da forma

$$
A=P_{1} \oplus P_{2} \oplus \ldots \oplus P_{n}
$$

onde cada $P_{i}=e_{i} A$ é um $A$-módulo projetivo indecomponível e $e_{1}, e_{2}, \ldots, e_{n}$ são idempotentes primitivos, dois a dois ortogonais, e tais que $1=e_{1}+e_{2}+$ $\ldots+e_{n}$. O conjunto $\left\{e_{1}, e_{2}, \ldots, e_{n}\right\}$ é chamado de um conjunto completo de idempotentes primitivos ortogonais.

Dizemos que $A$ é uma álgebra básica se $e_{i} A \varsubsetneqq e_{j} A$, quando $i \neq j$, para todo $i, j=1, \ldots, n$. De outro lado dizemos que $A$ é conexa (ou indecomponível) se não pode ser decomposta como soma direta de duas álgebras, ou equivalentemente, se 0 e 1 são seus únicos idempotentes centrais.

Denotamos por $\operatorname{Mod} A$ a categoria cujos objetos são os $A$-módulos à direita e cujos morfismos são os homomorfismos de $A$-módulos e por $\bmod A$ a subcategoria plena de $\operatorname{Mod} A$ cujos objetos são os $A$-módulos finitamente gerados.

Dado um $A$-módulo $M$, o $A$-módulo $M / \operatorname{rad} M$ é denotado por top $M$.

Se $M$ é um $A$-módulo, então existem seqüências exatas do tipo

$$
\cdots \longrightarrow P_{m} \stackrel{h_{m}}{\longrightarrow} P_{m-1} \longrightarrow \cdots \longrightarrow P_{1} \stackrel{h_{1}}{\longrightarrow} P_{0} \stackrel{h_{0}}{\longrightarrow} M \longrightarrow 0
$$

onde os $P_{j}$ são $A$-módulos projetivos. Uma tal seqüência é chamada uma resolução projetiva de $M$. Se $M$ admite uma resolução projetiva da forma

$$
0 \longrightarrow P_{m} \stackrel{h_{m}}{\longrightarrow} P_{m-1} \longrightarrow \cdots \longrightarrow P_{1} \stackrel{h_{1}}{\longrightarrow} P_{0} \stackrel{h_{0}}{\longrightarrow} M \longrightarrow 0,
$$


com $P_{m} \neq 0$, dizemos que o comprimento de tal resolução é $m$.

Se toda resolução projetiva de $M$ é infinita dizemos que $M$ tem dimensão projetiva infinita, caso contrário a dimensão projetiva de $M$, denotada por $p d M$, é o menor natural $\ell$ tal que existe uma resolução projetiva de $M$ de comprimento $\ell$.

A dimensão global à direita da $K$-álgebra $A$, que é denotada por gldim $A$, é definida por

$$
\operatorname{gldim} A=\sup \{p d M: \quad M \text { é um } A-\text { módulo à direita }\} .
$$

Para o caso em que $A$ é uma $K$-álgebra de dimensão finita, é conhecido que que sua dimensão global é para calcular sua dimensão global é

$\operatorname{gldim} A=\sup \{p d S: \quad S$ é um $A-$ módulo simples à direita $\}$.

\subsection{Carcases e álgebras de caminho}

Seja $A$ uma álgebra de dimensão finita e básica sobre um corpo algebricamente fechado. Nesta seção daremos uma caracterização de $A$ em termos de estruturas chamadas carcases. Para isto, começaremos definindo carcases, veremos como se podem construir álgebras a partir deles e para finalizar enunciaremos o Teorema de Gabriel, que proporciona a caracterização mencionada acima. A prova de tal teorema pode ser vista no Capítulo II de [4].

Um carcás é uma quádrupla $Q=\left(Q_{0}, Q_{1}, c, f\right)$ de dois conjuntos $Q_{0}$ (cujos elementos chamamos vértices) e $Q_{1}$ (cujos elementos chamamos flechas), e duas funções $c: Q_{1} \rightarrow Q_{0}$ e $f: Q_{1} \rightarrow Q_{0}$. 
Dada uma flecha $\alpha \in Q_{1}$, se $c(\alpha)=a$ e $f(\alpha)=b$ dizemos que o começo de $\alpha$ é $a$ e que o final de $\alpha$ é $b$. Denotamos esta situação por $\alpha: a \rightarrow b$. O carcás $Q=\left(Q_{0}, Q_{1}, c, f\right)$ pode ser denotado por $Q=\left(Q_{0}, Q_{1}\right)$ ou simplesmente por $Q$.

Dizemos que $Q=\left(Q_{0}, Q_{1}\right)$ é finito se os conjuntos $Q_{0}$ e $Q_{1}$ são finitos. Todos os carcases que consideraremos aqui serão finitos, exceto o carcás de Auslander-Reiten que definiremos na seção 1.4.

O grafo subjacente $\bar{Q}$ do carcás $Q$ é um grafo obtido de $Q$ sem considerar a orientação das flechas, isto é, $\bar{Q}$ é um grafo com os mesmos vértices de $Q$ e tal que existe uma aresta entre os vértices $a$ e $b$ em $\bar{Q}$ se existe uma flecha $\alpha: a \rightarrow b$ ou uma flecha $\beta: b \rightarrow a$. Dizemos que o carcás $Q$ é conexo se o grafo subjacente $\bar{Q}$ é conexo.

Sejam $Q=\left(Q_{0}, Q_{1}, c, f\right)$ e $a, b \in Q_{0}$. Um caminho de comprimento $\ell \geq 1$ com começo em $a$ e final em $b$ (ou simplesmente de $a$ para $b$ ) é uma seqüência

$$
\left(a\left|\alpha_{1}, \alpha_{2}, \ldots, \alpha_{\ell}\right| b\right)
$$

onde $\alpha_{k} \in Q_{1}$, para $1 \leq k \leq \ell, c\left(\alpha_{1}\right)=a, f\left(\alpha_{k}\right)=c\left(\alpha_{k+1}\right)$, para cada $1 \leq k \leq \ell-1$, e $f\left(\alpha_{\ell}\right)=b$. Denotamos tal caminho por $\alpha_{1} \alpha_{2} \ldots \alpha_{\ell}$. Além disso, a cada vértice $a \in Q_{0}$ associamos um caminho de comprimento $\ell=0$ que chamamos caminho trivial e que denotamos por $\epsilon_{a}$ ou por $(a \| a)$.

Dados um carcás $Q$ e um corpo $K$ a álgebra de caminhos $K Q$ é uma $K$-álgebra cujo $K$-espaço vetorial subjacente tem como base o conjunto de todos os caminhos de comprimento $\ell \geq 0$ em $Q$. A seguir definimos o produto em $K Q$ nos elementos de sua base. Se $\gamma_{1}=\left(a\left|\alpha_{1}, \alpha_{2}, \ldots, \alpha_{\ell}\right| b\right)$ e $\gamma_{2}=$ $\left(c\left|\beta_{1}, \beta_{2}, \ldots, \beta_{k}\right| d\right)$, então 


$$
\gamma_{1} \gamma_{2}=\left\{\begin{array}{l}
\left(a\left|\alpha_{1}, \alpha_{2}, \ldots, \alpha_{\ell}, \beta_{1}, \beta_{2}, \ldots, \beta_{k}\right| d\right), \text { se } b=c \\
0, \text { caso contrário. }
\end{array}\right.
$$

Tal produto é estendido a qualquer elemento de $K Q$ por linearidade.

Uma relação em $Q$ é uma combinação $K$-linear de caminhos de comprimento maior do que um que tem o mesmo início e o mesmo final. Isto é, uma relação $\rho$ é um elemento de $K Q$ da forma

$$
\rho=\sum_{i=1}^{m} \lambda_{i} \omega_{i},
$$

onde $\lambda_{i} \in K$ (não todos nulos), $\ell\left(\omega_{i}\right)>1, c\left(\omega_{i}\right)=c\left(\omega_{j}\right)$ e $f\left(\omega_{i}\right)=f\left(\omega_{j}\right)$.

Sejam $Q$ um carcás finito e $R$ o ideal de $K Q$ gerado pelas flechas de $Q$. Dizemos que um ideal bilateral $I$ de $K Q$ é admissível se existe um inteiro $m \geq 2$, tal que

$$
R^{m} \subseteq I \subseteq R^{2}
$$

Notemos que, se $Q$ é finito e $I$ é um ideal admissível de $K Q$, então existe um conjunto finito de relações $\left\{\rho_{1}, \rho_{2}, \ldots, \rho_{s}\right\}$ tais que $I=\left\langle\rho_{1}, \rho_{2}, \ldots, \rho_{s}\right\rangle$. O par $(Q, I)$ é chamado carcás com relações e a álgebra quociente $K Q / I$ associada ao par $(Q, I)$ é chamada de álgebra de caminhos com relações $(Q, I)$.

Até aqui dado um carcás $Q$ definimos a álgebra $K Q$ a partir de $Q$. Agora, assumindo que $A$ é uma $K$-álgebra de dimensão finita, básica e $K$ um corpo algebricamente fechado vamos construir o carcás $Q_{A}$ a partir de $A$ e veremos de que forma $A$ e $K Q_{A}$ estão relacionadas.

Seja $A$ uma álgebra básica com $\operatorname{dim}_{K} A<\infty, K$ algebricamente fechado e $\left\{e_{1}, e_{2}, \ldots, e_{n}\right\}$ um conjunto completo de idempotentes ortogonais primitivos. 
O carcás ordinário de $A$, que denotamos por $Q_{A}$, é definido da seguinte forma:

1. Os vértices de $Q_{A}$ são $v_{1}, v_{2}, \ldots, v_{n}$ que estão em correspondência bijetora com os idempotentes $e_{1}, e_{2}, \ldots, e_{n}$.

2. Dados dois vértices $v_{i}$ e $v_{j}$ em $\left(Q_{A}\right)_{0}$, fixamos uma base para o $K$ espaço vetorial $e_{i}\left(\operatorname{rad} A / \operatorname{rad}^{2} A\right) e_{j}$. As flechas $\alpha: v_{i} \rightarrow v_{j}$ estão em correspondência bijetora com os vetores de tal base.

A seguir enunciamos o Teorema de Gabriel que estabelece uma relação entre álgebras e seus carcases.

Teorema 1.2.1. (Gabriel) Seja A uma K-álgebra básica e conexa de dimensão finita, onde $K$ é um corpo algebricamente fechado. Então existe um ideal admissivel $I$ de $K Q_{A}$ tal que $A \cong K Q_{A} / I$. Além disso, se $\psi: K Q \rightarrow A$ é um epimorfismo com ker $\psi$ admissível, então $Q=Q_{A}$.

Um epimorfismo como no teorema acima se chama uma apresentação de $A$.

\subsection{Representações e módulos}

Na seção anterior, vimos como algumas álgebras podem ser descritas em termos de carcases com relações. Agora vamos visualizar as representações de uma álgebra, isto é, os seus módulos, através do correspondente carcás com relações. Todos os conceitos aqui apresentados podem ser encontrados no Capítulo III de [4]. 
Ao longo desta seção assumimos que $A$ é uma $K$-álgebra de dimensão finita, básica e $K$ um corpo algebricamente fechado e consideraremos em todos os casos carcases finitos.

Definimos uma representação $K$-linear ou, simplesmente, uma representação $M$ do carcás $Q$ da seguinte forma:

1. Para cada ponto $a \in Q_{0}$ associamos um $K$-espaço vetorial $M_{a}$.

2. Para cada flecha $\alpha: a \rightarrow b$ em $Q_{1}$ associamos uma aplicação $K$-linear $\varphi_{\alpha}: M_{a} \rightarrow M_{b}$

Denotamos tal representação como $M=\left(M_{a}, \varphi_{\alpha}\right)_{a \in Q_{0}, \alpha \in Q_{1}}$, ou simplesmente como $M=\left(M_{a}, \varphi_{\alpha}\right)$; e diremos que é de dimensão finita se cada espaço vetorial $M_{a}$ é de dimensão finita.

Sejam $M=\left(M_{a}, \varphi_{\alpha}\right)$ e $M^{\prime}=\left(M_{a}^{\prime}, \varphi_{\alpha}^{\prime}\right)$ duas representações de $Q$. Um morfismo (de representações) $f: M \rightarrow M^{\prime}$ é uma família $f=\left(f_{a}\right)_{a \in Q_{0}}$ de $K$ aplicações lineares $f_{a}: M_{a} \rightarrow M_{a}^{\prime}$, as quais são compatíveis com as aplicações $\varphi_{\alpha}$. Isto é, para cada flecha $\alpha: a \rightarrow b$, vale que

$$
\varphi_{\alpha}^{\prime} f_{a}=f_{b} \varphi_{\alpha}
$$

ou, equivalentemente, cada um dos seguintes quadrados comutam:

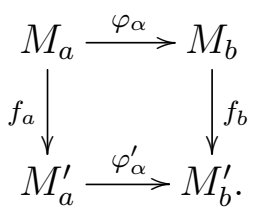


Sejam $f: M \rightarrow M^{\prime}$ e $g: M^{\prime} \rightarrow M^{\prime \prime}$ dois morfismos de representações de $Q$, onde $f=\left(f_{a}\right)_{a \in Q_{0}}$ e $g=\left(g_{a}\right)_{a \in Q_{0}}$. Definimos a composição $g f: M \longrightarrow M^{\prime \prime}$ como sendo a família $g f=\left(g_{a} f_{a}\right)_{a \in Q_{0}}$.

Desse modo, temos definido a categoria $\operatorname{Rep}(Q)$ de representações $K$ lineares de $Q$. Denotamos por $\operatorname{rep}(Q)$ a subcategoria plena de $\operatorname{Rep}(Q)$ que consiste das representações de dimensão finita.

Seja $M=\left(M_{a}, \varphi_{\alpha}\right)$ uma representação de $Q$. Para um caminho não trivial $\omega=\left(a\left|\alpha_{1}, \alpha_{2}, \ldots, \alpha_{\ell}\right| b\right)$ em $Q$, a avaliação de $M$ em $\omega$ é a função $K$-linear $\varphi_{\omega}: M_{a} \rightarrow M_{b}$ definida por $\varphi_{\omega}=\varphi_{\alpha_{1}} \varphi_{\alpha_{2}} \ldots \varphi_{\alpha_{\ell}}$. Estendemos esta definição para combinações lineares de caminhos. Isto é, se $\rho=\sum_{i=1}^{m} \lambda_{i} \omega_{i}$ é uma relação, então a avaliação de $M$ em $\rho$ é $\varphi_{\rho}=\sum_{i=1}^{m} \lambda_{i} \varphi_{\omega_{i}}$.

Seja $I$ um ideal admissível de $K Q$. Dizemos que a representação $M=\left(M_{a}, \varphi_{\alpha}\right)$ de $Q$ satisfaz as relações em $I$ quando $\varphi_{\rho}=0$, para todo elemento $\rho \in I$. Notemos que se $I$ é gerado pelo conjunto de relações $\left\{\rho_{1}, \rho_{2}, \ldots, \rho_{s}\right\}$, a representação $M$ satisfaz as relações de $I$ se, e somente se, $\varphi_{\rho_{i}}=0$, para todo $1 \leq i \leq s$.

Uma representação de $(Q, I)$ é uma representação de $Q$ que satisfaz as relações de $I$. Denotamos por $\operatorname{Rep}_{K}(Q, I)$ a subcategoria de $\operatorname{Rep}_{K}(Q)$ cujos objetos são as representações de $(Q, I)$ e por $\operatorname{rep}_{K}(Q, I)$ a subcategoria de $\operatorname{rep}_{K}(Q)$ cujos objetos são as representações de dimensão finita de $(Q, I)$.

Com as hipóteses sobre $A$ sabemos, pelo Teorema de Gabriel, que $A$ é isomorfa a uma álgebra de caminhos dada por um carcás com relações $(Q, I)$, isto é, $A \cong K Q / I$. A seguinte proposição diz que o estudo da categoria $M o d A$ é equivalente ao da categoria $\operatorname{Rep}(Q, I)$.

Teorema 1.3.1. Seja $A=K Q / I$, onde $Q$ é um carcás finito e conexo e $I$ é 
um ideal admissível de $K Q$. Então existe uma equivalência $F$ de categorias

$$
F: \operatorname{Mod} A \stackrel{\widetilde{\sim}}{\rightarrow} \operatorname{Rep}_{K}(Q, I)
$$

cuja restrição é uma equivalência entre as categorias

$$
F: \bmod A \stackrel{\widetilde{\sim}}{\rightarrow} \operatorname{rep}_{K}(Q, I) .
$$

Demonstração. $\quad$ Descreveremos o funtor $F: \operatorname{Mod} A \rightarrow \operatorname{Rep}_{K}(Q, I)$. Para isto diremos como age nos objetos e nos morfismos de $\operatorname{Mod} A$.

Sejam $a \in Q_{0}$ e $\alpha \in Q_{1}$. Denotaremos por $e_{a}$ e por $\bar{\alpha}$ as classes de $\epsilon_{a}$ e de $\alpha$ em $K Q / I$, respectivamente.

Se $M$ é um $A$-módulo, definimos $F(M)=\left(M_{a}, \varphi_{\alpha}\right)$, onde $M_{a}=M e_{a}$ e para $\alpha: a \rightarrow b$, seja $\varphi_{\alpha}: M_{a} \longrightarrow M_{b}$ dada por $\varphi_{\alpha}(x)=x \bar{\alpha}$, para todo $x \in M_{a}$.

Se $f: M \rightarrow M^{\prime}$ é um homomorfismo de $A$-módulos, então definimos $F(f)=\left(f_{a}\right)_{a \in Q_{0}}$, onde $f_{a}$ é a restrição de $f$ a $M_{a}=M e_{a}$. É fácil verificar que $F$ é uma equivalência de categorias.

A equivalência do teorema anterior permite a identificação dos $A$-módulos com as representações $K$-lineares de $(Q, I)$ e vice versa. Em vista deste fato abusaremos da linguagem não distinguindo, muitas vezes, os $K Q / I$ - módulos das representações de $(Q, I)$.

O último teorema tem muitas conseqüências interessantes, em particular permite conhecer de forma explícita os módulos simples, os projetivos indecomponíveis e os injetivos indecomponíveis. 


\subsection{Seqüências de Auslander-Reiten}

Neste seção vamos enunciar um resultado que surgiu na década dos 70 e que influenciou definitivamente o desenvolvimento da Teoria de Representações de álgebras. Trabalhando basicamente com álgebras de Artin (uma generalização das álgebras de dimensão finita) M. Auslander e I. Reiten introduziram a noção de seqüências quase-cindidas (também chamadas seqüências de Auslander-Reiten). A importância destas seqüências reside nos morfismos que as compõem.

Posteriormente os morfismos que aparecem nas mencionadas seqüências foram usados por C. M. Ringel para definir um carcás, conhecido como o carcás de Auslander-Reiten, que proporciona muita informação sobre a categoria $\bmod A$.

Detalhes dos conceitos aqui mencionados podem ser achados no Capítulo IV de [4].

Começaremos definindo os conceitos relacionados.

Sejam $M, N, L A$-módulos em $\bmod A$. Então:

1. Seja $h: M \rightarrow N$ um homomorfismo de $A$-módulos. Dizemos que $h$ é uma seção (ou um monomorfismo que cinde) se existe um homomorfismo de $A$-módulos $s: N \longrightarrow M$ tal que $s h=1_{M}$. De outro lado, dizemos que $h$ é uma retração (ou um epimorfismo que cinde) se existe um homomorfismo de $A$-módulos $r: N \longrightarrow M$ tal que $h r=1_{N}$.

2. Um homomorfismo de $A$-módulos $f: L \rightarrow M$ é chamado minimal à esquerda se cada $h \in \operatorname{End}_{A}(M)$ tal que $h f=f$ é um automorfismo. 
3. Um homomorfismo de $A$-módulos $g: M \rightarrow N$ é chamado minimal à direita se cada $k \in \operatorname{End}_{A}(M)$ tal que $g k=g$ é um automorfismo.

4. Um homomorfismo de $A$-módulos $f: L \rightarrow M$ é dito quase-cindido à esquerda se:

(a) $f$ não é seção.

(b) Para cada $A$-homomorfismo $u: L \rightarrow U$, que não é seção, existe $u^{\prime}: M \rightarrow U$ tal que $u^{\prime} f=u$.

5. Um homomorfismo de $A$-módulos $g: M \rightarrow N$ é dito quase-cindido à direita se:

(a) $g$ não é retração.

(b) Para cada $A$-homomorfismo $v: V \rightarrow N$, que não é retração, existe $v^{\prime}: V \rightarrow M$ tal que $g v^{\prime}=v$.

6. Um homomorfismo de $A$-módulos $f: L \rightarrow M$ é denominado minimal quase-cindido à esquerda se é minimal à esquerda e quase-cindido à esquerda.

7. Um homomorfismo de $A$-módulos $g: M \rightarrow N$ é denominado minimal quase-cindido à direita se é minimal à direita e quase-cindido à direita.

8. Um homomorfismo $h: M \longrightarrow N$ de $A$-módulos é irredutível se $h$ não é seção, nem retração e se $h=h_{1} h_{2}$ implica que $h_{1}$ é retração ou que $h_{2}$ é seção. 
Dizemos que uma seqüência exata curta

$$
0 \longrightarrow L \stackrel{f}{\longrightarrow} M \stackrel{g}{\longrightarrow} N \longrightarrow 0, \text { em } \bmod A
$$

é uma seqüência quase-cindida ou uma seqüência de Auslander-Reiten se $L$ e $N$ são $A$-módulos indecomponíveis e $f$ e $g$ são morfismos irredutíveis (ou equivalentemente, se $f$ é minimal quase cindido à esquerda e $g$ é minimal quase cindido à direita).

\section{Teorema 1.4.1. (Auslander-Reiten)}

1. Seja $M$ um A-módulo indecomponível não projetivo. Então existe uma seqüência quase-cindida, única a menos de equivalências de seqüências exatas, da forma

$$
0 \longrightarrow M^{\prime} \longrightarrow E \longrightarrow M \longrightarrow 0, \quad \operatorname{em} \bmod A \text {. }
$$

2. Seja L um A-módulo indecomponível não injetivo. Então existe uma seqüência quase-cindida, única a menos de equivalências de seqüências exatas, da forma

$$
0 \longrightarrow L \longrightarrow F \longrightarrow L^{\prime} \longrightarrow 0, \quad \operatorname{em} \bmod A \text {. }
$$

Sejam $X$ e $Y A$-módulos indecomponíveis. O $K$-espaço vetorial

$$
\operatorname{Irr}(X, Y)=\operatorname{rad}_{A}(X, Y) / \operatorname{rad}_{A}^{2}(X, Y)
$$

onde $\operatorname{rad}_{A}(X, Y)$ é o $K$-espaço vetorial dos homomorfismos não invertíveis de $X$ em $Y$ e $\operatorname{rad}_{A}^{2}(X, Y)$ é o $K$-espaço vetorial dos homomorfismos da forma 
gf com $f \in \operatorname{rad}_{A}(X, Z)$ e $g \in \operatorname{rad}_{A}(Z, Y)$, para algum $A$-módulo $Z$, é denominado espaço dos morfismos irredutíveis de $X$ em $Y$.

Como $X$ e $Y$ são $A$-módulos indecomponíveis, então é possível provar que a dimensão de $\operatorname{Irr}(X, Y)$ é igual ao número máximo de homomorfismos irredutíveis de $X$ em $Y$, que são linearmente independentes.

O carcás de Auslander-Reiten da categoria mod $A$, denotado por $\Gamma(\bmod A)$, é definido da seguinte forma:

1. Os vértices de $\Gamma(\bmod A)$ são as classes de isomorfismos $[M]$ de $A$ módulos indecomponíveis $M$.

2. Se $[M]$ e $[N]$ são dois vértices de $\Gamma(\bmod A)$, correspondentes aos $A$ módulos indecomponíveis $M$ e $N$, o número de flechas de $[M]$ para $[N]$ é igual à dimensão do espaço vetorial $\operatorname{Irr}(M, N)$. 


\section{Capítulo 2}

\section{Seqüências de Auslander-Reiten relativas}

M. Auslander e S. Smalø demonstraram em [7] que uma categoria funtorialmente finita, fechada por somandos diretos e por extensões tem seqüências quase-cindidas relativas. Neste capítulo definiremos as categorias $\mathcal{F}(\theta), \mathcal{X}(\theta)$, $\mathcal{Y}(\theta)$ e $\mathcal{W}(\theta)$, onde $\theta=\{\theta(1), \ldots, \theta(n)\}$ é um conjunto fixado de $A$-módulos tais que $\operatorname{Ext}_{A}^{1}(\theta(j), \theta(i))=0$, para todo $j \geq i$. Demonstraremos que $\mathcal{F}(\theta)$ é uma subcategoria funtorialmente finita de $\bmod A$ e, como conseqüência, que $\mathcal{X}(\theta)$ admite seqüências quase-cindidas relativas.

Todos os resultados aqui contidos foram apresentados por Ringel em [25].

Seja $A$ uma $K$-álgebra de dimensão finita. Fixamos o conjunto de $A$ módulos $\theta=\{\theta(1), \ldots, \theta(n)\}$ com a propriedade de que $\operatorname{Ext}_{A}^{1}(\theta(j), \theta(i))=0$, para todo $j \geq i$. Denotamos por $\mathcal{F}(\theta)$ a subcategoria plena de $\bmod A$ cujos objetos são os $A$-módulos à direita $M$ que admitem uma $\theta$-filtração, isto é, os $A$-módulos $M$ tais que existe uma cadeia de submódulos

$$
0=M_{0} \subseteq M_{1} \subseteq \ldots \subseteq M_{t}=M
$$


tais que $M_{i} / M_{i-1} \cong \theta(k)$, para algum $k \in\{1,2, \ldots, n\}$, para todo $i=1,2, \ldots, t$.

Denotamos por $\mathcal{X}(\theta)$ a subcategoria plena de $\bmod A$ cujos objetos são os $A$-módulos que são somandos diretos de módulos em $\mathcal{F}(\theta)$.

Definição 2.1.1. Seja $\mathcal{X}$ uma subcategoria plena de mod A. Dizemos que $\mathcal{X}$ é fechada por somandos diretos se para cada módulo $X \in \mathcal{X}$ todos os somandos diretos de $X$ estão em $\mathcal{X}$. De outro lado, dizemos que $\mathcal{X}$ é fechada por extensões se para qualquer seqüência exata $0 \longrightarrow X_{1} \longrightarrow M \longrightarrow X_{2} \longrightarrow 0$, com $X_{1} \in X$ e $X_{2} \in \mathcal{X}$, vale que $M \in \mathcal{X}$.

Observação 2.1.2. Fixado o conjunto $\theta$ nas condições acima, temos que a categoria $\mathcal{F}(\theta)$ é fechada por extensões e que a categoria $\mathcal{X}(\theta)$ é fechada por somandos diretos e por extensões.

De fato, seja $0 \longrightarrow M \stackrel{\mu}{\longrightarrow} N \stackrel{\rho}{\longrightarrow} L \longrightarrow 0$ uma seqüência exata em $\bmod A$ tal que $M, L \in \mathcal{F}(\triangle)$. Se $0=M_{0} \subseteq M_{1} \subseteq M_{2} \subseteq \cdots \subseteq M_{t}=M$ e $0=L_{0} \subseteq$ $L_{1} \subseteq L_{2} \subseteq \cdots \subseteq L_{k}=L$ são $\theta$-filtrações de $M$ e $L$, respectivamente, então

$$
0 \subseteq \mu\left(M_{1}\right) \subseteq \cdots \subseteq \mu\left(M_{t}\right) \subseteq \rho^{-1}\left(L_{1}\right) \subseteq \cdots \subseteq \rho^{-1}\left(L_{k-1}\right) \subseteq \rho^{-1}\left(L_{k}\right)=N
$$

é uma $\theta$-filtração para $N$.

A categoria $\mathcal{X}(\theta)$ é fechada por somandos diretos e por extensões diretamente da sua definição.

Por outro lado $\mathcal{F}(\theta) \subseteq \mathcal{X}(\theta)$, mas em geral não coincidem como mostramos no seguinte exemplo. 
Exemplo 2.1.3. Sejam $K$ um corpo algebricamente fechado e $A=K Q$, onde $Q$ é o carcás

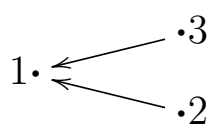

Os $A$-módulos projetivos indecomponíveis são os módulos associados às representações:

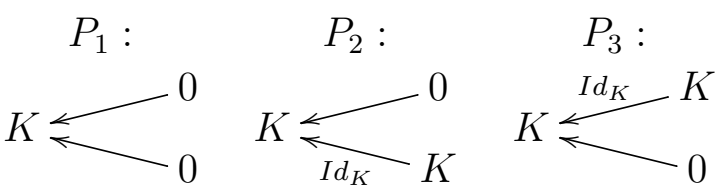

e os $A$-módulos injetivos indecomponíveis são os módulos associados às representações:
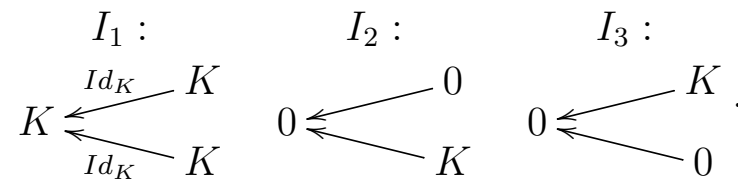

Mais ainda, $\operatorname{rad} P_{2}=\operatorname{rad} P_{3}=P_{1}=S_{1}$.

Seja $\theta=\left\{\theta_{1}, \theta_{2}\right\}$, onde $\theta_{1}=I_{1}$ e $\theta_{2}=P_{1}$. Como $\theta_{2}$ é projetivo, então $\operatorname{Ext}_{A}^{1}\left(\theta_{2}, \theta_{1}\right)=0$. As cadeias $0 \subset S_{1} \subset P_{2}$ e $0 \subset S_{1} \subset P_{3}$ são séries de composição para $P_{2}$ e $P_{3}$, respectivamente. Portanto $P_{2} \notin \mathcal{F}(\theta)$ e $P_{3} \notin \mathcal{F}(\theta)$. Mas $0 \subset S_{1} \subset P_{2} \oplus P_{3}$ é uma $\theta$-filtração de $P_{2} \oplus P_{3}$, pois $\left(P_{2} \oplus P_{3}\right) / S_{1} \cong I_{1}$. Logo $P_{2} \oplus P_{3} \in \mathcal{F}(\theta)$ e, em conseqüência, $P_{2} \in \mathcal{X}(\theta)$ e $P_{3} \in \mathcal{X}(\theta)$.

Definição 2.1.4. Sejam $\mathcal{X}$ uma subcategoria plena de $\bmod A$ e $M \in \bmod A$.

1. Uma $\mathcal{X}$-aproximação à direita de $M$ é um homomorfismo $\gamma: X \rightarrow M$, com $X \in \mathcal{X}$, tal que para todo homomorfismo $\gamma^{\prime}: X^{\prime} \rightarrow M$, com $X^{\prime} \in \mathcal{X}$, existe um homomorfismo $\varepsilon: X^{\prime} \rightarrow X$ que torna comutativo 
o seguinte diagrama

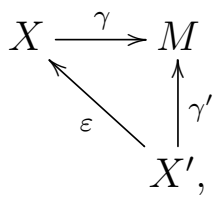

isto é, existe um $\varepsilon$ tal que $\gamma^{\prime}=\gamma \varepsilon$. Dualmente, definimos uma $\mathcal{X}$ aproximação à esquerda de $M$ como um homomorfismo $\gamma: M \rightarrow X$, com $X \in \mathcal{X}$, tal que para todo homomorfismo $\gamma^{\prime}: M \rightarrow X^{\prime}$, com $X^{\prime} \in \mathcal{X}$, existe um homomorfismo $\varepsilon: X \rightarrow X^{\prime}$ que torna comutativo o seguinte diagrama

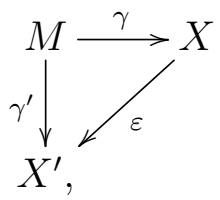

isto é, existe um $\varepsilon$ tal que $\gamma^{\prime}=\varepsilon \gamma$.

2. Dizemos que a categoria $\mathcal{X}$ é contravariantemente finita $e m \bmod A$ se todo $M$ em $\bmod A$ admite uma $\mathcal{X}$-aproximação à direita. Dualmente, dizemos que $\mathcal{X}$ é covariantemente finita $\mathrm{em} \bmod A$ se todo $M$ em $\bmod A$ admite uma $\mathcal{X}$-aproximação à esquerda. Finalmente, dizemos que $\mathcal{X}$ é uma categoria funtorialmente finita $\mathrm{em} \bmod A$ quando é covariantemente finita e contravariantemente finita em mod A.

Definição 2.1.5. Seja $\mathcal{X}$ uma subcategoria plena de mod A. Denotamos por $\mathcal{Y}_{\mathcal{X}}$ a subcategoria dos módulos $Y \in \bmod A$ tais que $\operatorname{Ext}_{A}^{1}(X, Y)=0$, para todo $X \in \mathcal{X}$.

Lema 2.1.6. Seja $M \in \bmod A$ e suponhamos que existe uma seqüência exata

$$
0 \longrightarrow Y \longrightarrow X \stackrel{\gamma}{\longrightarrow} M \longrightarrow 0
$$


com $X \in \mathcal{X}$ e $Y \in \mathcal{Y}_{\mathcal{X}}$. Então $\gamma$ é uma $\mathcal{X}$-aproximação à direita de $M$.

Demonstração. Seja $\gamma^{\prime}: X^{\prime} \longrightarrow M$ um homomorfismo com $X^{\prime} \in \mathcal{X}$. Construímos o diagrama de pull-back

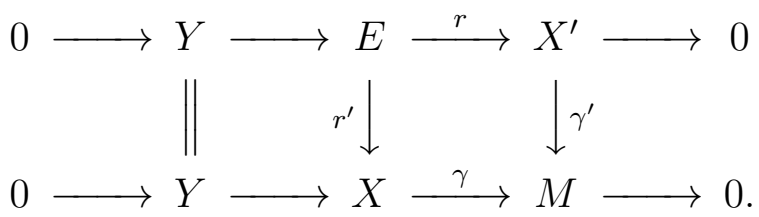

Como $\operatorname{Ext}_{A}^{1}\left(X^{\prime}, Y\right)=0$, pois $X^{\prime} \in \mathcal{X}$ e $Y \in \mathcal{Y}_{\mathcal{X}}$, então $r$ é um epimorfismo que cinde e, portanto, existe $t: X^{\prime} \rightarrow E$ tal que o seguinte diagrama comuta

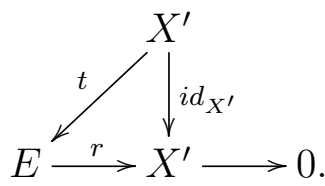

Seja $\epsilon=r^{\prime} t: X^{\prime} \longrightarrow X$. Pela comutatividade do diagrama acima, temos que, $\gamma \epsilon=\gamma^{\prime}$. Logo $\gamma$ é uma $\mathcal{X}$-aproximação à direita de $M$.

Lema 2.1.7. Seja $\mathcal{X}$ uma subcategoria de $\bmod A$ fechada por extensões e tal que para todo $N \in \bmod A$ existe uma seqüência exata

$$
0 \longrightarrow N \longrightarrow Y^{(N)} \longrightarrow X^{(N)} \longrightarrow 0
$$

$\operatorname{com} X^{(N)} \in \mathcal{X}$ e $Y^{(N)} \in \mathcal{Y}_{\mathcal{X}}$. Então todo módulo $M$ em mod $A$ admite uma $\mathcal{X}$-aproximação à direita.

Demonstração. Seja $M \in \bmod A$. Consideramos primeiramente o caso em existe um epimorfismo $\pi: X \longrightarrow M \operatorname{com} X \in \mathcal{X}$. Seja $P=K e r \pi$. Por 
hipótese, existe uma seqüência exata $0 \longrightarrow P \longrightarrow Y^{(P)} \longrightarrow X^{(P)} \longrightarrow 0$, com $Y^{(P)} \in \mathcal{Y}_{\mathcal{X}}$ e $X^{(P)} \in \mathcal{X}$. Se

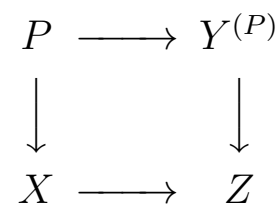

é o push-out de $X \longleftarrow P \longrightarrow Y^{(P)}$, então temos o diagrama comutativo

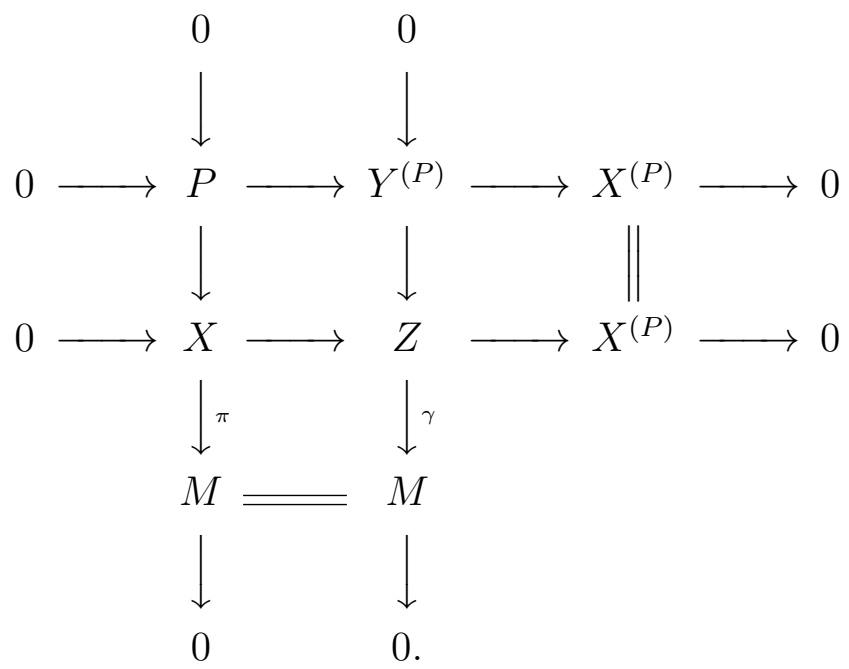

Como $X$ e $X^{(P)}$ estão em $\mathcal{X}$ e $\mathcal{X}$ é fechada por extensões, então $Z \in \mathcal{X}$. De outro lado, aplicando o lema anterior à seqüência da segunda coluna do diagrama, temos que $\gamma: Z \longrightarrow M$ é uma $\mathcal{X}$-aproximação à direita de $M$.

Para o caso geral consideramos o $A$-módulo $M^{\prime}$ gerado pelas imagens $\operatorname{Im} \phi$, onde $\phi: W \longrightarrow M$, para todo $W \in \mathcal{X}$. O módulo $M^{\prime}$ é conhecido como o traço de $\mathcal{X}$ em $M$. Sob as nossas hipóteses, existe um número finito $m$ de morfismos $\pi_{i}: W_{i} \rightarrow M$, com $W_{i} \in \mathcal{X}$, tais que as imagens de $\pi_{i}$ geram $M^{\prime}$. Como $\mathcal{X}$ é uma categoria fechada por somas diretas (pois é fechada por extensões), então temos que $X=\oplus_{i=1}^{m} W_{i} \in \mathcal{X}$ e o homomorfismo $\psi: X \longrightarrow M^{\prime}$ definido por $\psi\left(x_{1}+\ldots+x_{n}\right)=\pi_{1}\left(x_{1}\right)+\ldots+\pi_{m}\left(x_{m}\right)$ é um 
epimorfismo. Portanto, pela primeira parte, existe uma $\mathcal{X}$-aproximação à direita de $M^{\prime}, \gamma^{\prime}: Z \longrightarrow M^{\prime}$. Se $i: M^{\prime} \longrightarrow M$ é a inclusão, então $i \gamma^{\prime}$ : $Z \longrightarrow M$ é uma $\mathcal{X}$-aproximação à direita de $M$.

Seja $A$ uma $K$-álgebra de dimensão finita e fixemos agora um conjunto $\theta=\{\theta(1), \ldots, \theta(n)\}$ de $A$-módulos à direita, com a propriedade de que $\operatorname{Ext}_{A}^{1}(\theta(j), \theta(i))=0$, para todo $j \geq i$.

Observemos que se $\mathcal{X}=\mathcal{F}(\theta)$, então podemos caracterizar $\mathcal{Y}_{\mathcal{X}}$ da Definição 2.1.5, que denotamos por $\mathcal{Y}(\theta)$, como a subcategoria plena de $\bmod A$ formada pelos módulos $Y$ tais que $\operatorname{Ext}_{A}^{1}(\theta(j), Y)=0$, para todo $1 \leq j \leq n$. Para o conjunto $\theta$ fixado temos o seguintes lemas úteis para o objetivo do capítulo.

Lema 2.1.8. Dado um $t \in\{1,2, \ldots, n\}$, seja $N$ um A-módulo tal que, para todo $j>t, \operatorname{Ext}_{A}^{1}(\theta(j), N)=0$. Então existe uma seqüência exata

$$
0 \longrightarrow N \longrightarrow N_{t} \longrightarrow Q_{t} \longrightarrow 0
$$

onde $Q_{t}$ é uma soma de cópias de $\theta(t)$ e $\operatorname{Ext}_{A}^{1}\left(\theta(j), N_{t}\right)=0$, para todo $j \geq t$.

Demonstração. Para cada $t$ sejam

$$
\epsilon_{s}=\left(0 \longrightarrow N \stackrel{f_{s}}{\longrightarrow} T_{s} \stackrel{g_{s}}{\longrightarrow} \theta(t) \longrightarrow 0\right),
$$

para $1 \leq s \leq m$, as seqüências exatas tais que as correspondentes classes de equivalências $\left[\epsilon_{1}\right], \ldots,\left[\epsilon_{m}\right]$ geram o espaço $\operatorname{Ext}_{A}^{1}(\theta(t), N)$. Consideremos o 
seguinte diagrama comutativo:

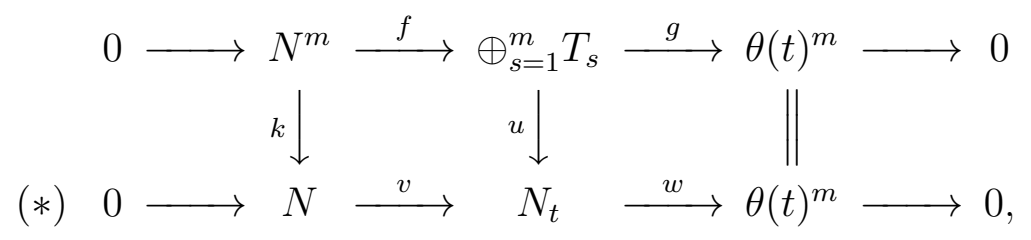

onde

$$
f=\left[\begin{array}{lll}
f_{1} & & 0 \\
& \ddots & \\
0 & & f_{m}
\end{array}\right], g=\left[\begin{array}{lll}
g_{1} & & 0 \\
& \ddots & \\
0 & & g_{m}
\end{array}\right], k=[1,1, \ldots, 1]
$$

e o quadrado

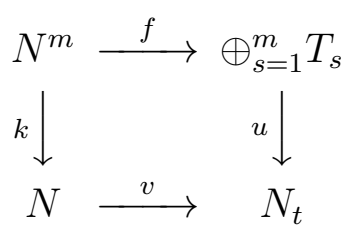

é o push-out de $N \stackrel{k}{\leftarrow} N^{m} \stackrel{f}{\rightarrow} \oplus_{s=1}^{m} T_{s}$.

Vamos mostrar que a seqüência (*) é a seqüência desejada. Primeiramente, mostraremos que para cada $s, 1 \leq s \leq m$, vale que $\epsilon_{s}=\operatorname{Ext}_{A}^{1}\left(u_{s}, A\right) \epsilon$, onde $u_{s}: \theta(t) \rightarrow \theta(t)^{m}$ é a inclusão natural na $s$-ésima coordenada e $\epsilon$ é o elemento de $\operatorname{Ext}_{A}^{1}\left(\theta(t)^{m}, N\right)$ representado pela seqüência $(*)$. Para isto, consideramos o diagrama abaixo, que é comutativo

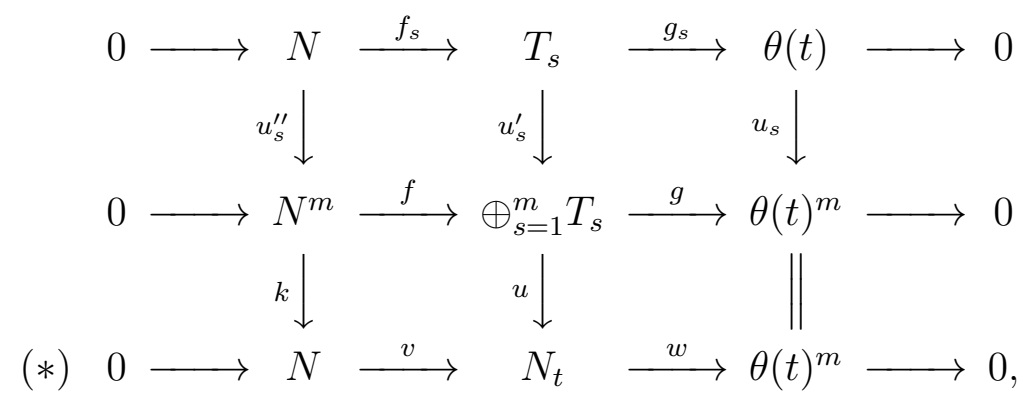

onde $u_{s}^{\prime}$ e $u_{s}^{\prime \prime}$ indicam as respectivas inclusões na $s$-ésima coordenada. Desde 
que $k u_{s}^{\prime \prime}=1_{N}$, resulta que é comutativo o seguinte diagrama:

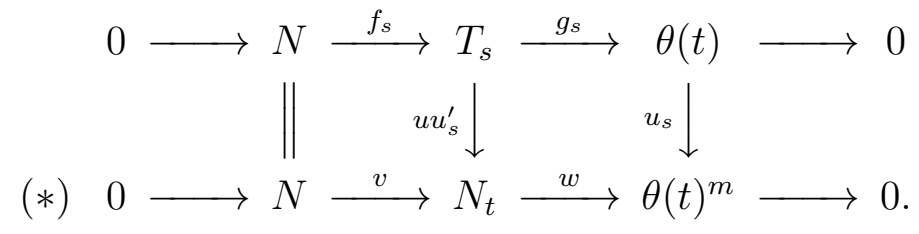

E a afirmação sobre cada $\epsilon_{s}$ está verificada.

Vamos mostrar agora que $\operatorname{Ext}_{A}^{1}\left(\theta(j), N_{t}\right)=0$, para cada $j \geq t$. Para tanto, apliquemos o funtor $\operatorname{Hom}_{A}(\theta(j), \ldots)$ para $j \geq t$ à seqüência $(*)$. Como $\operatorname{Ext}_{A}^{1}\left(\theta(j), \theta(t)^{m}\right)=0$, para cada $j \geq t$, obtemos a seqüência exata:

$$
(* *) \operatorname{Hom}_{A}\left(\theta(t), \theta(t)^{m}\right) \stackrel{\delta_{t}}{\longrightarrow} \operatorname{Ext}_{A}^{1}(\theta(t), N) \longrightarrow \operatorname{Ext}_{A}^{1}\left(\theta(t), N_{t}\right) \longrightarrow 0,
$$

para $j \geq t$. Desde que, por hipótese, $\operatorname{Ext}_{A}^{1}(\theta(t), N)=0$, para cada $j>t$, segue imediatamente da seqüência $(* *)$ que $\operatorname{Ext}_{A}^{1}\left(\theta(t), N_{t}\right)=0$, para cada $j>t$.

Vejamos então para $j=t$. Como visto acima $\epsilon_{s}=\operatorname{Ext}_{A}^{1}\left(u_{s}, A\right) \epsilon=\delta_{t}\left(u_{s}\right)$, ou seja que os elementos geradores do espaço $\operatorname{Ext}_{A}^{1}(\theta(t), N)$ estão na imagem do homomorfismo de conexão $\delta_{t}$, o que implica que $\delta_{t}$ é sobrejetor. Logo, pela seqüência $(* *)$, temos que $\operatorname{Ext}_{A}^{1}\left(\theta(t), N_{t}\right)=0$. Portanto, $\operatorname{Ext}_{A}^{1}\left(\theta(j), N_{t}\right)=0$, para cada $j \geq t$.

Lema 2.1.9. Dado um $t \in\{1,2, \ldots, n\}$, seja $N$ um A-módulo tal que, para todo $j>t, \operatorname{Ext}_{A}^{1}(\theta(j), N)=0$. Então existe uma seqüência

$$
0 \longrightarrow N \longrightarrow Y \longrightarrow X \longrightarrow 0
$$

$\operatorname{com} X \in \mathcal{F}(\theta(1), \ldots, \theta(t))$ e $Y \in \mathcal{Y}(\theta)$. 
Demonstração. Seja $N$ um $A$-módulo tal que $\operatorname{Ext}_{A}^{1}(\theta(j), N)=0$, para todo $j>t$. Então, pelo lema anterior, existem um $A$-módulo $Q_{t} \cong \theta(t)^{\alpha_{t}}$, para algum inteiro $\alpha_{t} \geq 0$, e uma seqüência exata

$$
0 \longrightarrow N=N_{t+1} \stackrel{\mu_{t}}{\longrightarrow} N_{t} \longrightarrow Q_{t} \longrightarrow 0
$$

onde $N_{t}$ é tal que $\operatorname{Ext}_{A}^{1}\left(\theta(j), N_{t}\right)=0$, para $j \geq t$. Da mesma forma existe uma seqüência exata

$$
0 \longrightarrow N_{t} \stackrel{\mu_{t-1}}{\longrightarrow} N_{t-1} \longrightarrow Q_{t-1} \longrightarrow 0
$$

onde $Q_{t-1} \cong \theta(t-1)^{\alpha_{t-1}}$ e $N_{t-1}$ é tal que $\operatorname{Ext}_{A}^{1}\left(\theta(j), N_{t-1}\right)=0$, para $j \geq t+1$. Dessa forma construímos indutivamente uma família de seqüências:

$$
0 \longrightarrow N_{i} \stackrel{\mu_{i-1}}{\longrightarrow} N_{i-1} \longrightarrow Q_{i-1} \longrightarrow 0,
$$

com $\operatorname{Ext}_{A}^{1}\left(\theta(j), N_{i}\right)=0$, para todo $j \geq i$, e $Q_{i-1} \cong \theta(i-1)^{\alpha_{i-1}}$. Assim obtemos a cadeia de monomorfismos

$$
N_{t+1} \stackrel{\mu_{t}}{\longrightarrow} N_{t} \stackrel{\mu_{t-1}}{\longrightarrow} \cdots \stackrel{\mu_{1}}{\longrightarrow} N_{1}
$$

Sejam $\mu=\mu_{1} \ldots \mu_{t}: N \rightarrow N_{1}, Y=N_{1}$ e $X=\operatorname{coker}(\mu)$. Então obtemos seqüência exata

$$
0 \longrightarrow N \stackrel{\mu}{\longrightarrow} Y \longrightarrow X \longrightarrow 0
$$

que satisfaz as propriedades requeridas. De fato, pois $Y=N_{1} \in \mathcal{Y}(\theta)$ e como na cadeia de inclusões $N_{t+1} / N \subseteq N_{t} / N \subseteq \ldots N_{2} / N \subseteq N_{1} / N=X$ vale que $\left(N_{i} / N\right) /\left(N_{i+1} / N\right) \cong N_{i} / N_{i+1} \cong Q_{i}$, para todo $1 \leq i \leq t$, então $X \in \mathcal{F}(\theta(1), \ldots, \theta(t))$. 
No lema anterior, o caso $t=n$ é de particular interesse e o formulamos a seguir.

Lema 2.1.10. Seja $N \in \bmod A$. Então existe uma seqüência exata

$$
0 \longrightarrow N \longrightarrow Y \longrightarrow X \longrightarrow 0,
$$

$\operatorname{com} X \in \mathcal{F}(\theta)$ e $Y \in \mathcal{Y}(\theta)$.

Com a posse das definições e resultados anteriores, estamos em condições de apresentar o resultado principal deste capítulo.

Teorema 2.1.11. A subcategoria $\mathcal{F}(\theta)$ é funtorialmente finita em $\bmod A$.

Demonstração. O Lema 2.1.10 garante a existência de seqüências do tipo

$$
0 \longrightarrow N \longrightarrow Y \longrightarrow X \longrightarrow 0
$$

com $X \in \mathcal{F}(\theta)$ e $Y \in \mathcal{Y}(\theta)$, para cada $A$-módulo $N$ em $\bmod A$. Por outro lado, podemos concluir pelo Lema 2.1.7 que todo $A$-módulo em $\bmod A$ admite uma $\mathcal{F}(\theta)$-aproximação à direita, isto é, que $\mathcal{F}(\theta)$ é contravariantemente finita em $\bmod A$. De outro lado, como as versões duais dos lemas anteriores são válidas, concluímos que $\mathcal{F}(\theta)$ é também uma categoria covariantemente finita em $\bmod A$.

Se $\mathcal{X}$ é uma categoria plena de $\bmod A$ arbitrária denotamos por $\mathcal{W}_{\mathcal{X}}$ a subcategoria plena de $\bmod A$ formada pelos módulos $W$ tais que $\operatorname{Ext}_{A}^{1}(W, X)=$ 0, para todo $X \in \mathcal{X}$. Em particular, se $\mathcal{X}=\mathcal{F}(\theta)$ podemos caracterizar $\mathcal{W}_{\mathcal{X}}$, 
que neste caso denotamos por $\mathcal{W}(\theta)$, como a subcategoria plena de $\bmod A$ dos módulos $W$ tais que $\operatorname{Ext}_{A}^{1}(W, \theta(j))=0$, para todo $1 \leq j \leq n$.

Proposição 2.1.12. As categorias $\mathcal{Y}(\theta)$ e $\mathcal{W}(\theta)$ são contravariantemente finita e covariantemente finita em mod $A$, respectivamente.

Demonstração. Só mostraremos a primeira afirmação, pois a segunda tem demonstração dual. Em virtude do Lema 2.1.10, temos que para cada $A$-módulo $N$ existe uma seqüência exata $0 \longrightarrow N \stackrel{\beta}{\longrightarrow} Y \longrightarrow X \longrightarrow 0$, com $X \in \mathcal{F}(\theta)$ e $Y \in \mathcal{Y}(\theta)$. De forma dual a como foi feito na demonstração do Lema 2.1.6 é possível mostrar que $\beta$ é uma $\mathcal{Y}(\theta)$-aproximação de $N$.

Como foi comentado antes, uma conseqüência importante deste teorema é que $\mathcal{X}(\theta)$ tem seqüências quase-cindidas relativas. Descrevemos o que isto significa de forma precisa nas definições que seguem, e que podem ser encontradas em [7].

Definição 2.1.13. Sejam $\mathcal{C}$ uma subcategoria de $\bmod A$ e $X$ um A-módulo em $\mathcal{C}$. Dizemos que $X$ é Ext-projetivo ou relativamente projetivo em $\mathcal{C}$ se $\operatorname{Ext}_{A}^{1}(X, Y)=0$, para todo $Y$ em $\mathcal{C}$. De forma dual, dizemos que $X$ $e ́$ Ext-injetivo ou relativamente injetivo em $\mathcal{C}$ se $\operatorname{Ext}_{A}^{1}(Y, X)=0$, para todo $Y$ em $\mathcal{C}$.

Definição 2.1.14. Seja $\mathcal{C}$ uma subcategoria de $\bmod A$. Dizemos que:

1. Um homomorfismo de A-módulos $f: L \rightarrow M$ em $\mathcal{C}$ é quase-cindido à esquerda $\operatorname{em~} \mathcal{C}$ se:

(a) f ñ̃o é um monomorfismo que cinde. 
(b) Para cada A-homomorfismo $u: L \rightarrow U$ em $\mathcal{C}$, que não é um monomorfismo que cinde, existe $u^{\prime}: M \rightarrow U$ em $\mathcal{C}$ tal que $u^{\prime} f=u$; isto é, existe um $u^{\prime}$ que torna comutativo o seguinte diagrama:

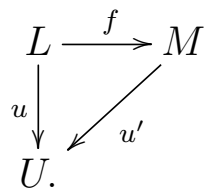

2. Um homomorfismo de A-módulos $g: M \rightarrow N$ em $\mathcal{C}$ é quase-cindido à direita em $\mathcal{C}$ se:

(a) f não é um epimorfismo que cinde.

(b) Para cada A-homomorfismo $v: V \rightarrow N$ em $\mathcal{C}$, que não é um epimorfismo que cinde, existe $v^{\prime}: V \rightarrow M$ em $\mathcal{C}$ tal que $g v^{\prime}=v$; isto é, existe um $v^{\prime}$ que torna comutativo o seguinte diagrama:

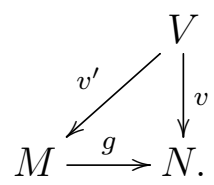

3. $\mathcal{C}$ admite homomorfismos quase-cindidos à direita se para cada módulo indecomponíveil $C$ em $\mathcal{C}$ existe um módulo $B$ em $\mathcal{C}$ e um homomorfismo $f: B \rightarrow C$ que é quase-cindido à direita em $\mathcal{C} . \quad$ De forma dual, dizemos que $\mathcal{C}$ admite homomorfismos quase-cindidos à esquerda quando para todo módulo indecomponível $C$ em $\mathcal{C}$ existe um módulo $D$ em $\mathcal{C}$ e um homomorfismo $g: C \rightarrow D$ que é quasecindido à esquerda. Finalmente dizemos que $\mathcal{C}$ admite homomorfismos quase-cindidos se admite tanto homomorfismos quase-cindidos 
à esquerda quanto homomorfismos quase-cindidos à direita.

4. $\mathcal{C}$ admite seqüências quase-cindidas ou que admite seqüências de Auslander-Reiten relativas se:

(a) $\mathcal{C}$ admite homomorfismos quase-cindidos.

(b) Para todo módulo indecomponível $C$ em $\mathcal{C}$, que não é Ext-projetivo, existe uma seqüência exata

$$
0 \longrightarrow A \stackrel{f}{\longrightarrow} B \stackrel{g}{\longrightarrow} C \longrightarrow 0
$$

com $f$ quase-cindida à esquerda em $\mathcal{C}$ e g quase-cindida à direita em $\mathcal{C}$.

(c) Para todo módulo indecomponível $A$ em $\mathcal{C}$, que não é Ext-injetivo, existe uma seqüência exata

$$
0 \longrightarrow A \stackrel{f}{\longrightarrow} B \stackrel{g}{\longrightarrow} C \longrightarrow 0
$$

com $f$ quase-cindida à esquerda em $\mathcal{C}$ e g quase-cindida à direita em $\mathcal{C}$.

Como conseqüência dos resultados anteriores temos a seguinte proposição.

Proposição 2.1.15. A categoria $\mathcal{X}(\theta)$ admite seqüências de Auslander-Reiten relativas.

Demonstração. Seja $M \in \bmod A$. Como $\mathcal{F}(\theta)$ é funtorialmente finita, pelo Teorema 2.1.11, consideremos $\gamma: X \longrightarrow M$ uma $\mathcal{F}(\theta)$-aproximação à direita de $M$. Vamos mostrar que $\gamma$ é na verdade uma $\mathcal{X}(\theta)$-aproximação à direita de $M$. Claramente $X \in \mathcal{X}(\theta)$. Seja $\delta: Y \longrightarrow M$ um homomorfismo 
qualquer em $\bmod A$, com $Y \in \mathcal{X}(\theta)$. Da definição de $\mathcal{X}(\theta)$, temos que existe um módulo $Y^{\prime}$ tal que $Y \oplus Y^{\prime} \in \mathcal{F}(\theta)$. Seja o homomorfismo $\delta \pi: Y \oplus$ $Y^{\prime} \longrightarrow M$, onde $\pi$ denota a projeção canônica de $Y \oplus Y^{\prime}$ sobre $Y$. Como $\gamma$ é uma $\mathcal{F}(\theta)$-aproximação à direita de $M$, existe $\epsilon_{1}: Y \oplus Y^{\prime} \longrightarrow X$ tal que $\gamma \epsilon_{1}=\delta \pi$. Seja $\epsilon=\epsilon_{1} i$, onde $i$ é a inclusão natural de $Y$ em $Y \oplus Y^{\prime}$. Temos pois que $\gamma \epsilon=\delta$. Logo $\gamma$ é uma $\mathcal{X}(\theta)$-aproximação à direita de $M$. Além disso, como $\mathcal{X}(\theta)$ é fechada por extensões e por somandos diretos, por [7] (Teorema 2.4), resulta que $\mathcal{X}(\theta)$ admite seqüências quase-cindidas relativas. 


\section{Capítulo 3}

\section{Álgebras estandarmente estratificadas e álgebras quase hereditárias}

As álgebras quase-hereditárias foram introduzidas por E. Cline, B. Parshall e L. Scott, em [9] e [10], no contexto da teoria algébrica de grupos e da representação das álgebras de Lie complexas, semisimples de dimensão finita, mais precisamente no estudo da categoria dos pesos máximos. Mais tarde em [11] os mesmos autores introduziram um conceito mais geral: as álgebras estandarmente estratificadas.

As álgebras quase-hereditárias não são nada mais do que as álgebras estandarmente estratificadas de dimensão global finita. O principal exemplo, onde elas aparecem, é a categoria $\mathcal{O}$ de Berstein, Gelfand e Gelfand associada com a decomposição triangular de uma álgebra de Lie complexa, semisimples de dimensão finita. Um outro exemplo são as chamadas álgebras de Auslander.

O conceito central nesta teoria são os módulos estandares e coestandares. 
Tais módulos dependem de forma essencial da ordem fixada para o conjunto de módulos simples.

Os resultados aqui apresentados estão contidos em [13], [15], [26] e [24].

Ao longo deste capítulo $K$ denotará um corpo algebricamente fechado, $A$ uma $K$-álgebra básica, conexa e com dimensão finita sobre $K$. Além disso, $\bmod A$ denotará a categoria dos $A$-módulos à direita finitamente gerados.

\subsection{Sobre o grupo de Grothendieck}

É de nosso interesse o estudo dos fatores de composição de módulos de comprimento finito. Para isto estudaremos o grupo de Grothendieck, que é um grupo bastante especial sob este ponto de vista, pois ele contém toda informação a respeito dos fatores de composição da categoria de módulos finitamente gerados. Os resultados apresentados nesta seção poderam ser encontrados em [4].

Seja $A$ uma $K$-álgebra e $\left\{e_{1}, e_{2}, \ldots, e_{n}\right\}$ um conjunto completo de idempotentes primitivos ortogonais de $A$. Se $A=\oplus_{i=1}^{n} e_{i} A$, então os $A$-módulos $P_{i}=e_{i} A$ e $I_{i}=D\left(A e_{i}\right), i=1, \ldots, n$, denotam, respectivamente, a cobertura projetiva e a envolvente injetiva do simples $S_{i} \cong$ top $P_{i} \cong \operatorname{Soc} I_{i}$.

Lembramos que, dado $M \in \bmod A$ e um elemento idempotente $e \in A$, vale que $\operatorname{Hom}_{A}(e A, M) \cong M e$. Em particular $\operatorname{End}_{A}\left(S_{i}\right) \cong e_{i}($ top $A) e_{i}$.

Seja $\boldsymbol{e}=\left(e_{1}, e_{2}, \ldots, e_{n}\right)$ um conjunto completo e ordenado de idempotentes primitivos ortogonais de $A$. A ordem fixada $\boldsymbol{e}$ determina uma ordenação no conjunto dos $A$-módulos simples $S_{i}$, bem como no de suas coberturas projetivas e envolventes injetivas. 
Definição 3.1.1. Seja $M \in \bmod A$. Definimos o vetor dimensão de $M$ como vetor de $\mathbb{Z}^{n}$, denotado por $\underline{\operatorname{dim}} M$, dado por

$$
\underline{\operatorname{dim}} M=\left[\begin{array}{c}
\operatorname{dim}_{K} M e_{1} \\
\vdots \\
\operatorname{dim}_{K} M e_{n}
\end{array}\right],
$$

onde $\left\{e_{1}, \ldots, e_{n}\right\}$ é um conjunto completo de idempotentes primitivos ortogonais de $A$.

Assim o vetor $\underline{\operatorname{dim}} S_{i}$, de cada simples $S_{i}$, é o $i$-ésimo vetor da base canônica de $\mathbb{Z}^{n}$. De outro lado, como temos que $\operatorname{Hom}_{A}\left(P_{i}, M\right) \cong M e_{i}$ e que $D \operatorname{Hom}_{A}\left(M, I_{i}\right) \cong D \operatorname{Hom}_{A^{o p}}\left(A e_{i}, M\right) \cong D\left(e_{i} D M\right) \cong D(D M) e_{i} \cong M e_{i}$, podemos reescrever o vetor $\underline{\operatorname{dim}} M$ das seguintes formas:

$$
\underline{\operatorname{dim}} M=\left[\begin{array}{c}
\operatorname{dim}_{K} \operatorname{Hom}_{A}\left(P_{1}, M\right) \\
\vdots \\
\operatorname{dim}_{K} \operatorname{Hom}_{A}\left(P_{n}, M\right)
\end{array}\right]=\left[\begin{array}{c}
\operatorname{dim}_{K} \operatorname{Hom}_{A}\left(M, I_{1}\right) \\
\vdots \\
\operatorname{dim}_{K} \operatorname{Hom}_{A}\left(M, I_{n}\right)
\end{array}\right] .
$$

Observação 3.1.2. Se $0 \longrightarrow L \longrightarrow M \longrightarrow N \longrightarrow 0$ é uma seqüência exata em $\bmod A$, então $\underline{\operatorname{dim}} M=\underline{\operatorname{dim}} L+\underline{\operatorname{dim}} N$.

Definição 3.1.3. Seja A uma K-álgebra, básica e de dimensão finita sobre K. Chama-se grupo de Grothendieck de $\bmod A$ o grupo abeliano $K_{0}(A)=\mathcal{F} / \mathcal{F}^{\prime}$, onde $\mathcal{F}$ é o grupo abeliano livre cuja base é o conjunto de classes de isomorfismos $\tilde{M}$ dos módulos $M$ em $\bmod A$ e $\mathcal{F}^{\prime}$ é o subgrupo de $\mathcal{F}$ gerado pelos elementos $\tilde{M}-\tilde{L}-\tilde{N}$ correspondentes às seqüências exatas $0 \longrightarrow L \longrightarrow M \longrightarrow N \longrightarrow 0 \mathrm{em} \bmod A$.

Denotaremos por $[M]$ a imagem da classe de isomorfismo $\tilde{M}$ do módulo $M$ pelo epimorfismo canônico de grupos $\mathcal{F} \longrightarrow \mathcal{F} / \mathcal{F}^{\prime}$ e por $\left[M: S_{i}\right]$ ao número de fatores de composição de $M$ isomorfos a $S_{i}$. 
A proposição abaixo fornece uma caracterização do grupo $K_{0}(A)$, cuja demonstração pode ser encontrada em [4].

Proposição 3.1.4. Sejam A uma K-álgebra, básica, de dimensão finita sobre $K e\left\{S_{1}, \ldots, S_{n}\right\}$ um conjunto completo de classes de isomorfismo de $A$ módulos simples à direita. Então, o grupo de Grothendieck $K_{0}(A)$ de $\bmod A$ é um grupo abeliano livre com uma base dada por $\left\{\left[S_{1}\right], \ldots,\left[S_{n}\right]\right\}$ e existe um único isomorfismo de grupos dado por

$$
\underline{\operatorname{dim}}: K_{0}(A) \longrightarrow \mathbb{Z}^{n}
$$

tal que, $\underline{\operatorname{dim}}([M])=\underline{\operatorname{dim}} M$ para todo A-módulo $M$.

Corolário 3.1.5. Seja $M$ um A-módulo. Então, para cada $j=1, \ldots, n$, vale que

$$
\left[M: S_{j}\right]=\operatorname{dim}_{K} \operatorname{Hom}_{A}\left(P_{j}, M\right)=\operatorname{dim}_{K} \operatorname{Hom}_{A}\left(M, I_{j}\right) .
$$

Em particular, usando os vetores dimensão dos $A$-módulos projetivos (ou injetivos) indecomponíveis obtemos uma matriz de coeficientes inteiros, que é conhecida como matriz de Cartan de $A$.

Definição 3.1.6. Sejam A uma K-álgebra, básica de dimensão finita sobre $K$ $e\left\{e_{1}, \ldots, e_{n}\right\}$ um conjunto completo de idempotentes primitivos e ortogonais de A. A matriz de Cartan de A é a matriz $n \times n$

$$
C_{A}=\left[\begin{array}{ccc}
c_{11} & \cdots & c_{1 n} \\
\vdots & \ddots & \vdots \\
c_{1 n} & \cdots & c_{n n}
\end{array}\right]
$$


onde $c_{j i}=\operatorname{dim}_{K} e_{j} A e_{i}$ para $i, j=1, \ldots, n$.

Observemos que, como $e_{j} A e_{i} \cong \operatorname{Hom}_{A}\left(P_{i}, P_{j}\right) \cong \operatorname{Hom}_{A}\left(I_{i}, I_{j}\right)$, cada entrada $c_{j i}$ de $C_{A}$ corresponde ao número de homomorfismos linearmente independentes de $P_{i}$ a $P_{j}$ e ao número de homomorfismos linearmente independentes de $I_{i}$ a $I_{j}$.

\subsection{Módulos estandares e coestandares}

Introduziremos aqui os conceitos fundamentais de módulos estandares e módulos coestandares. Caracterizaremos estes módulos através dos seus fatores de composição e apresentaremos algumas de suas propriedades básicas, que podem ser consultadas em [15].

Sejam $A$ uma $K$-álgebra e $\boldsymbol{e}=\left(e_{1}, e_{2}, \ldots, e_{n}\right)$ um conjunto ordenado e completo de idempotentes ortogonais e primitivos de $A$.

Para cada $i, 1 \leq i \leq n$, denotamos por $\varepsilon_{i}$ o idempotente

$$
\varepsilon_{i}=e_{i}+e_{i+1}+\ldots+e_{n}
$$

e definimos $\varepsilon_{n+1}=0$.

Das observações da seção anterior, temos que são válidos os isomorfismos

$$
\oplus_{j=i}^{n} P_{j} \cong \varepsilon_{i} A \quad \text { e } \operatorname{End}_{\mathrm{A}}\left(\varepsilon_{\mathrm{i}} \mathrm{A}\right) \cong \varepsilon_{\mathrm{i}} \mathrm{A} \varepsilon_{\mathrm{i}} \cong \operatorname{End}_{\mathrm{A}}\left(\mathrm{A} \varepsilon_{\mathrm{i}}\right) .
$$

Definição 3.2.1. Dados os A-módulos $X$ e $Y$, definimos o traço de $Y$ em $X$, que denotamos por $\tau_{Y}(X)$, como o submódulo de $X$ gerado pelas imagens dos homomorfismos de $Y$ em $X$, isto é, $\tau_{Y}(X)=\left\langle\operatorname{Im} \varphi: \varphi \in \operatorname{Hom}_{A}(Y, X)\right\rangle$.

Observação 3.2.2. Sejam $f \in A$ um idempotente e $X \in \bmod A$. Então

$$
\tau_{f A}(X)=X f A .
$$


De fato, sejam $f \in A$ um idempotente e $\varphi \in \operatorname{Hom}_{A}(f A, X)$. Então $\varphi(f a)=\varphi\left(f^{2} a\right)=\varphi(f) f a \in X f A$, para todo $a \in A$. Além disso, se $x \in X$ e $\varphi_{x} \in \operatorname{Hom}_{A}(f A, X)$ é tal que $\varphi_{x}(f a)=x f a$, então $\operatorname{Im} \varphi_{x}=x f A$ e portanto $<\operatorname{Im} \varphi_{x}: x \in X>=X f A$.

Em particular $\tau_{P_{i}}(X)=X e_{i} A$ e $\tau_{\varepsilon_{i} A}(X),=X \varepsilon_{i} A$, para cada $i=1,2, \ldots, n$.

Daqui em diante, para facilitar a escrita, denotaremos por $U_{i}$ o módulo $\tau_{\varepsilon_{i+1} A}\left(P_{i}\right)$ e para um módulo $X \in \bmod A$ qualquer denotamos por $X^{(i)}$ o módulo $\tau_{\varepsilon_{i} A}(X)$.

Enunciaremos o seguinte Lema que é bem útil.

Lema 3.2.3. Sejam $Y, Z$ em $\bmod A$. Então

1. $\tau_{Y}(Z)$ é o submódulo maximal de $Z$ tal que existe um epimorfismo $\xi: Y^{m} \longrightarrow \tau_{Y}(Z)$, com $m \geq 1$.

2. Se $\operatorname{Hom}_{A}\left(P_{j}, Z / Z^{(k)}\right) \neq 0$, então $j<k$.

\section{Demonstração.}

1. Seja $\left\{f_{1}, f_{2}, \ldots, f_{m}\right\}$ uma base de $\operatorname{Hom}_{A}(Y, Z)=\operatorname{Hom}_{A}\left(Y, \tau_{Y}(Z)\right)$ como $K$-espaço vetorial. Afirmamos que o homomorfismo $\xi: Y^{m} \longrightarrow \tau_{Y}(Z)$ definido por $\xi\left(y_{1}, \cdots, y_{m}\right)=f_{1}\left(y_{1}\right)+\ldots+f_{m}\left(y_{m}\right)$ é um epimorfismo. De fato, se $x \in \tau_{Y}(Z)$, então existem $f \in \operatorname{Hom}_{A}(Y, Z)$ e $y \in Y$ tais que $f(y)=x$. Mas $f=\sum_{i=1}^{m} \lambda_{i} f_{i}, \operatorname{logo} x=f(y)=\sum_{i=1}^{m} \lambda_{i} f_{i}(y)=$ $\sum_{i=1}^{m} f_{i}\left(\lambda_{i} y\right)=\xi\left(\lambda_{1} y_{1}, \ldots, \lambda_{m} y_{m}\right)$. Claramente $\tau_{Y}(Z)$ é maximal com relação à mencionada propriedade. 
2. Seja $j \geq k$. Consideremos a seqüência exata

$$
0 \longrightarrow Z^{(k)} \stackrel{i}{\longrightarrow} Z \stackrel{\pi}{\longrightarrow} Z / Z^{(k)} \longrightarrow 0
$$

onde $i$ e $\pi$ são a inclusão e a projeção canônica, respectivamente, e apliquemos o funtor $\operatorname{Hom}_{A}\left(P_{j}, \_\right)$. Como $\operatorname{Ext}_{A}^{1}\left(P_{j}, M\right)=0$, obtemos a seguinte seqüência exata

$$
0 \longrightarrow \operatorname{Hom}_{A}\left(P_{j}, Z^{(k)}\right) \longrightarrow \operatorname{Hom}_{A}\left(P_{j}, Z\right) \longrightarrow \operatorname{Hom}_{A}\left(P_{j}, Z / Z^{(k)}\right) \longrightarrow 0 .
$$

Portanto, se $f \in \operatorname{Hom}_{A}\left(P_{j}, Z / Z^{(k)}\right)$, então existe $g \in \operatorname{Hom}_{A}\left(P_{j}, Z\right)$ tal que $\pi g=f$. Mas $I m g \subseteq \operatorname{ker} \pi=Z^{(k)}, \operatorname{logo} \pi g=f=0$.

Definição 3.2.4. Seja $\boldsymbol{e}=\left(e_{1}, e_{2}, \ldots, e_{n}\right)$ uma ordem fixada de um conjunto ordenado e completo de idempotentes ortogonais e primitivos $\left\{e_{1}, e_{2}, \ldots, e_{n}\right\}$ de A. A seqüência $\triangle=\left(\triangle_{1}, \triangle_{2}, \ldots, \triangle_{n}\right)$ de módulos estandares à direita, com respeito à ordem e, é dada por

$$
\triangle_{i}=P_{i} / \tau_{\varepsilon_{i+1} A}\left(P_{i}\right)=P_{i} / e_{i} A \varepsilon_{i+1} A \cong e_{i} A / e_{i} A \varepsilon_{i+1} A, \quad \text { (Observação 3.2.2) }
$$

A seqüência $\triangle^{o}=\left(\triangle_{1}^{o}, \triangle_{2}^{o}, \ldots, \triangle_{n}^{o}\right)$ de A-módulos estandares à esquerda está dada por $\triangle_{i}^{o} \cong A e_{i} / A \varepsilon_{i+1} A e_{i}$ e a seqüência $\nabla=\left(\nabla_{1}, \nabla_{2}, \ldots, \nabla_{n}\right)$ dos $A$-módulos coestandares à direita por $\nabla_{i}=\operatorname{Hom}_{K}\left(\triangle_{i}^{o}, K\right)=D\left(\triangle_{i}^{o}\right)$.

Da definição acima concluímos que, independentemente da ordem fixada, $\triangle_{n}$ é um $A$-módulo projetivo e $\nabla_{n}$ é um $A$-módulo injetivo.

O seguinte lema caracteriza os módulos estandares e coestandares em termos de seus fatores de composição. 
Lema 3.2.5. Para cada $i=1,2, \ldots, n, \triangle_{i}$ é o módulo quociente maximal de $P_{i}$ tal que se $\left[\triangle_{i}: S_{j}\right] \neq 0$ então $j \leq i$. Dualmente, $\nabla_{i}$ é o submódulo maximal de $I_{i}$ tal que se $\left[\nabla_{i}: S_{j}\right] \neq 0$ então $j \leq i$.

Demonstração. Provaremos a primeira afirmação, pois a segunda é a sua dual. O fato de que os fatores de composição de $\triangle_{i}$ são $S_{j} \operatorname{com} j \leq i$ decorre do Corolário 3.1.5 e do Lema 3.2.3.

Suponhamos que $N \subseteq P_{i}$ é um $A$-módulo tal que $\left[P_{i} / N: S_{k}\right] \neq 0$ implica que $k \leq i$ e que $j>i$. Desde que a sequência

$$
0 \longrightarrow \operatorname{Hom}_{A}\left(P_{j}, N\right) \longrightarrow \operatorname{Hom}_{A}\left(P_{j}, P_{i}\right) \longrightarrow \operatorname{Hom}_{A}\left(P_{j}, P i / N\right) \longrightarrow 0
$$

é exata e, pelo Corolário 3.1.5, temos que $\operatorname{Hom}_{A}\left(P_{j}, P_{i} / N\right)=0$, então $\operatorname{Hom}_{A}\left(P_{j}, N\right) \cong \operatorname{Hom}_{A}\left(P_{j}, P_{i}\right)$. Isto ultimo significa que se $\phi \in \operatorname{Hom}_{A}\left(P_{j}, P_{i}\right)$, então existe $h \in \operatorname{Hom}_{A}\left(P_{j}, N\right)$ tal que $\phi=i h$. Portanto $\operatorname{Im} \phi=\operatorname{Im} i h \subseteq N$, o que nos permite concluir que $U_{i} \subseteq N$.

Observação 3.2.6. O Corolário 3.1.5 e o Lema 3.2.5 garantem que, para $i=1,2, \ldots, n, \operatorname{Hom}_{A}\left(P_{i}, \nabla_{j}\right)=0$ se $j<i$ e que $\operatorname{Hom}_{A}\left(\triangle_{j}, I_{i}\right)=0$ se $j<i$.

Vejamos o lema abaixo que será útil para estabelecer algumas relações entre a seqüência dos módulos estandares e a seqüência dos módulos coestandares.

Lema 3.2.7. Seja $X$ em $\bmod A$. Então:

1. Se $\operatorname{Hom}_{A}\left(\triangle_{i}, X\right) \neq 0$, então $\left[X: S_{i}\right] \neq 0$.

2. Se $\operatorname{Ext}_{A}^{1}\left(\triangle_{i}, X\right) \neq 0$, então $\left[X: S_{k}\right] \neq 0$ para algum $k>i$. 


\section{Demonstração.}

1. Seja $\psi \in \operatorname{Hom}_{A}\left(\triangle_{i}, X\right), \psi \neq 0$. Se $\pi: P_{i} \rightarrow \triangle_{i}$ é a projeção canônica, então $\psi \pi \in \operatorname{Hom}_{A}\left(P_{i}, X\right)$ e $\psi \pi \neq 0$, pois $\pi$ é um epimorfismo. Então, de acordo com o Corolário 3.1.5, $S_{i}$ é um fator de composição de $X$.

2. Se aplicamos o funtor $\operatorname{Hom}_{A}(\ldots, X)$ à seqüência exata

$$
0 \longrightarrow U_{i} \longrightarrow P_{i} \longrightarrow \triangle_{i} \longrightarrow 0
$$

obtemos a seqüência exata longa

$$
\cdots \longrightarrow \operatorname{Hom}_{A}\left(U_{i}, X\right) \longrightarrow \operatorname{Ext}_{A}^{1}\left(\triangle_{i}, X\right) \longrightarrow \operatorname{Ext}_{A}^{1}\left(P_{i}, X\right) \longrightarrow \cdots
$$

Como $\operatorname{Ext}_{A}^{1}\left(P_{i}, X\right)=0$ e $\operatorname{Ext}_{A}^{1}\left(\triangle_{i}, X\right) \neq 0$, então $\operatorname{Hom}_{A}\left(U_{i}, X\right) \neq$ 0. Seja $\phi \in \operatorname{Hom}_{A}\left(U_{i}, X\right)$, com $\phi \neq 0$. Pelo Lema 3.2.3 existe um epimorfismo $\xi:\left(\bigoplus_{l=i+1}^{n} P_{l}\right)^{m} \rightarrow U_{i}$ e portanto $\phi \xi \neq 0$. Logo existe um homomorfismo $\xi_{k}: P_{k} \rightarrow X$, com $\xi_{k} \neq 0$, para algum $i+1 \leq k \leq n$.

Portanto $S_{k}$ é fator de composição de $X$ para algum $k>i$.

Como conseqüência obtemos as seguintes propriedades.

\section{Proposição 3.2.8. (Propriedades dos módulos estandares e coestan- dares)}

1. $\operatorname{Hom}_{A}\left(\triangle_{i}, \triangle_{j}\right)=0$ se $j<i$.

2. $\operatorname{Ext}_{A}^{1}\left(\triangle_{i}, \triangle_{j}\right)=0$ se $j \leq i$.

3. $\operatorname{Hom}_{A}\left(\triangle_{i}, \nabla_{j}\right) \neq 0$ se, e somente se, $j=i$. 
4. $\operatorname{Ext}_{A}^{1}\left(\triangle_{i}, \nabla_{j}\right)=0$, para todo $1 \leq i, j \leq n$.

\section{Demonstração.}

1. Decorre do Lema 3.2.7 (1) $\operatorname{com} X=\triangle_{j}$.

2. Suponhamos que $\operatorname{Ext}_{A}^{1}\left(\triangle_{i}, \triangle_{j}\right) \neq 0$. Pelo Lema 3.2.7 (2) o módulo $S_{k}$ é fator de composição de $\triangle_{j}$, para algum $k>i$. Logo, pelo Lema 3.2.5, resulta que $k \leq j$ e portanto $i<j$.

3. Suponhamos que $\operatorname{Hom}_{A}\left(\triangle_{i}, \nabla_{j}\right) \neq 0$. Seja $\epsilon \in \operatorname{Hom}_{A}\left(\triangle_{i}, \nabla_{j}\right)$, com $\epsilon \neq$ 0. Como $\operatorname{Soc} \operatorname{Im}(\epsilon) \subseteq \operatorname{Soc} \nabla_{j}=S_{j}$, segue que $\operatorname{Soc} \operatorname{Im}(\epsilon)=S_{j}$. Assim $S_{j}$ é fator de composição de $\triangle_{i}$, e portanto, pelo Lema 3.2.5, $j \leq i$. De outro lado $S_{i}=$ top $\triangle_{i}$ é um fator de composição de $\nabla_{j}$ e portanto $i \leq j$, pelo Lema 3.2.5. Para a recíproca, notese que $\triangle_{i} / \mathrm{rad} \triangle_{i} \cong S_{i}$ e que a composta de $\pi: \triangle_{i} \longrightarrow \triangle_{i} / \operatorname{rad} \triangle_{i}$ e $i: S_{i} \longrightarrow \nabla$, onde $\pi$ e $i$ são a projeção e a inclusão canônica, respectivamente, é não nula.

4. Para $j \leq i$, aplicamos $\operatorname{Hom}_{A}\left(\ldots, \nabla_{j}\right)$ à $(3.1)$ e obtemos

$$
\cdots \longrightarrow \operatorname{Hom}_{A}\left(U_{i}, \nabla_{j}\right) \longrightarrow \operatorname{Ext}_{A}^{1}\left(\triangle_{i}, \nabla_{j}\right) \longrightarrow \operatorname{Ext}_{A}^{1}\left(P_{i}, \nabla_{j}\right) \longrightarrow \cdots
$$

Como $\operatorname{Ext}_{A}^{1}\left(P_{i}, \nabla_{j}\right)=0$, basta provar que $\operatorname{Hom}_{A}\left(U_{i}, \nabla_{j}\right)=0$, e para isto se procede da mesma forma que no Lema 3.2.7(2).

Para $j>i$, aplicando $\operatorname{Hom}_{A}(\ldots, K)$ à seqüência de $A$-módulos à direita $0 \longrightarrow A \varepsilon_{j+1} A e_{j} \longrightarrow A e_{j} \longrightarrow \triangle_{j}^{o} \longrightarrow 0$, obtemos a seqüência de $A$ módulos à esquerda

$$
0 \longrightarrow D\left(\triangle_{j}^{o}\right) \longrightarrow D\left(A e_{j}\right) \longrightarrow D\left(A \varepsilon_{j+1} A e_{j}\right) \longrightarrow 0
$$


que pode ser reescrita na forma

$$
0 \longrightarrow \nabla_{j} \longrightarrow I_{j} \longrightarrow V_{j} \longrightarrow 0
$$

pois $D\left(\triangle_{j}^{o}\right)=\nabla_{j}$ e $I_{j} \cong D\left(A e_{j}\right)$. Para concluir, se procede de forma análoga ao caso anterior aplicando $\operatorname{Hom}_{A}\left(\triangle_{i}, \ldots\right)$ em (3.2).

Observação 3.2.9. Os módulos $\triangle_{i}$, para $1 \leq i \leq n$, são indecomponíveis.

De fato, desde que cada $A$-módulo projetivo indecomponível $P_{i}=e_{i} A$ tem topo $S_{i}$, que é um $A$-módulo simples, segue que top $\triangle_{i}=\operatorname{top}\left(P_{i} / U_{i}\right)=S_{i}$ é simples e, por isso, cada $\triangle_{i}$ é indecomponível.

\subsection{As categorias $\mathcal{F}(\triangle)$ e $\mathcal{F}(\nabla)$}

Sejam $\triangle$ e $\nabla$ as sequências de módulos estandares e coestandares, respectivamente, para uma ordem fixada $\boldsymbol{e}=\left(e_{1}, e_{2}, \ldots, e_{n}\right)$ de um conjunto completo de idempotentes primitivos e ortogonais da $K$-álgebra $A$.

Apresentaremos nesta seção algumas propriedades básicas das categorias $\mathcal{F}(\triangle)$ e $\mathcal{F}(\nabla)$. Em particular, que elas são funtorialmente finitas, que admitem seqüências de Auslander-Reiten relativas, que $\mathcal{F}(\triangle)$ é uma categoria resolvente e que $\mathcal{F}(\nabla)$ é uma categoria corresolvente.

Observamos que os resultados aqui contidos se encontram em [24], [12] e $[26]$.

Com as notações introduzidas no Capítulo 2, recordemos que $\mathcal{F}(\triangle)$ denota a subcategoria plena de $\bmod A$ cujos objetos são os módulos que têm 
uma $\triangle$-filtração e que $\mathcal{F}(\nabla)$ denota subcategoria plena de $\bmod A$ cujos objetos são os módulos que têm uma $\nabla$-filtração.

Os módulos em $\mathcal{F}(\triangle)$ são chamamos de $\triangle$-bons módulos e os módulos em $\mathcal{F}(\nabla)$ de $\nabla$-bons módulos.

Definição 3.3.1. Seja $M \in \bmod A$. A cadeia de submódulos de $M$

$$
0=\tau_{\varepsilon_{n+1} A}(M) \subseteq \tau_{\varepsilon_{n} A}(M) \subseteq \ldots \subseteq \tau_{\varepsilon_{2} A}(M) \subseteq \tau_{\varepsilon_{1} A}(M)=M
$$

é denominada filtração traço de $M$ com respeito a ordem $\boldsymbol{e}$.

A filtração da definição anterior, com a convenção adotada na Seção 3.2 em que $M^{(i)}=\tau_{\varepsilon_{i} A}(M)$, pode ser reescrita na forma:

$$
0=M^{(n+1)} \subseteq M^{(n)} \subseteq \ldots \subseteq M^{(2)} \subseteq M^{(1)}=M
$$

A proposição que segue dá uma caracterização dos módulos em $\mathcal{F}(\triangle)$, e sua demonstração pode ser encontrada em [12].

Proposição 3.3.2. Um A-módulo $M \in \mathcal{F}(\triangle)$ se, e somente se, para todo $i=1,2, \ldots, n, M^{(i)} / M^{(i+1)} \cong \triangle_{i}^{t_{i}}$, para algum $t_{i} \geq 0$, onde $\triangle_{i}^{0}=0$.

Observação 3.3.3. Se $M \in \mathcal{F}(\triangle)$, então os módulos $M^{(t)}$ e $M / M^{(t)}$, para $1 \leq t \leq n$, estão em $\mathcal{F}(\triangle)$. Mais ainda, $M^{(t)}$ está filtrado por $\triangle_{j}, \operatorname{com} j \geq t$, e $M / M^{(t)}$ está filtrado por $\triangle_{j}$, com $j<t$.

$$
\text { Isto é } M^{(t)} \in \mathcal{F}\left(\left\{\triangle_{t}, \ldots, \triangle_{n}\right\}\right) \text { e } M / M^{(t)} \in \mathcal{F}\left(\left\{\triangle_{1}, \ldots, \triangle_{t-1}\right\}\right) \text {. }
$$

Antes de enunciar o seguinte corolário lembremos que um módulo nulo sobre qualquer anel $A$, é sempre um módulo projetivo. 
Corolário 3.3.4. Seja a $K$-álgebra $B_{i}=A / A \varepsilon_{i+1} A$. Então

1. $M \in \mathcal{F}(\triangle)$ se, e somente se, $M^{(i)} / M^{(i+1)}$ é $B_{i}$-módulo projetivo, para todo $i=1,2, \ldots, n$.

2. $M \in \mathcal{F}(\nabla)$ se, e somente se, $M^{(i)} / M^{(i+1)}$ é $B_{i}$-módulo injetivo, para todo $i=1,2, \ldots, n$.

Demonstração. Provaremos a primeira afirmação, pois a prova da segunda é dual. Observemos que $B_{i}$ se pode escrever da forma

$$
B_{i} \cong \bigoplus_{j=1}^{i} e_{j} A / e_{j} A \varepsilon_{i+1} A,
$$

onde o último somando é $\triangle_{i}$. Assim $\triangle_{i}$ é um $B_{i}$-módulo projetivo.

Se $M \in \mathcal{F}(\triangle)$, então pela proposição anterior o quociente $M^{(i)} / M^{(i+1)} \cong$ $\triangle_{i}^{t_{i}}$, para algum $t_{i} \geq 0$, e portanto é um $B_{i}$-módulo projetivo.

Reciprocamente suponhamos, para um $i$ fixo, que o quociente $M^{(i)} / M^{(i+1)}$ é $B_{i}$-módulo projetivo. Pelo Lema 3.2.3 existe um epimorfismo de $A$-módulos $\psi=\left(\psi_{1}, \ldots, \psi_{n}\right):\left(\bigoplus_{j=i}^{n} P_{j}^{t_{j}}\right) \rightarrow M^{(i)}$. Compondo $\psi$ com a projeção canônica $\pi: M^{(i)} \longrightarrow M^{(i)} / M^{(i+1)}$ obtemos o epimorfismo $\pi \psi=\left(\pi \psi_{1}, \ldots, \pi \psi_{n}\right)$ : $\left(\bigoplus_{j=i}^{n} P_{j}^{t_{j}}\right) \longrightarrow M^{(i)} / M^{(i+1)}$. Em virtude do Lema 3.2 .3 (2) resulta que os morfismos $\pi \psi_{j}: P_{j}^{t_{j}} \longrightarrow M^{(i)} / M^{(i+1)}$, para $j>i$, são nulos e, portanto, que $\pi \psi_{i}$ é um epimorfismo. Usando de novo o Lema 3.2.3 (2), obtemos que $\tau_{\varepsilon_{i+1} A}\left(P_{i}\right)=e_{i} A \varepsilon_{i+1} A \subset \operatorname{ker} \pi \psi_{i}$. A anterior inclusão induz um epimorfismo de $A$-módulos

$$
\bar{\psi}:\left(P_{i} / e_{i} A \varepsilon_{i+1} A\right)^{t_{i}} \longrightarrow M^{(i)} / M^{(i+1)} .
$$

Desde que $e_{i} A \varepsilon_{i+1} A \subset A \varepsilon_{i+1} A \subset A n n_{A}\left(M^{(i)} / M^{(i+1)}\right)$, então o homomorfismo $\bar{\psi}$ é também um epimorfismo de $B_{i}$-módulos. Como $M^{(i)} / M^{(i+1)}$ é 
$B_{i}$-projetivo, temos que $M^{(i)} / M^{(i+1)} \cong\left(P_{i} / e_{i} A \varepsilon_{i+1} A\right)^{t_{0}}=\triangle_{i}^{t_{0}}$, para algum $0<t_{0} \leq t_{i}$

Corolário 3.3.5. As subcategorias $\mathcal{F}(\triangle)$ e $\mathcal{F}(\nabla)$ são fechadas por somandos diretos.

Demonstração. Seja $B_{i}=A / A \varepsilon_{i+1} A$. Se $M_{1} \oplus M_{2} \in \mathcal{F}(\triangle)$, então, pelo corolário anterior, temos que $\left(M_{1} \oplus M_{2}\right)^{(i)} /\left(M_{1} \oplus M_{2}\right)^{(i+1)}$ é um $B_{i}$-módulo projetivo, para cada $i=1,2, \ldots, n$. Mas desde que $\left(M_{1} \oplus M_{2}\right)^{(i)}=M_{1}^{(i)} \oplus M_{2}^{(i)}$ ([19], Cap. 2, Proposição 8.22$)$ e $M_{j}^{(i)} \subset M_{j}^{(i+1)}$, para $j=1,2$, temos que

$$
\left(M_{1} \oplus M_{2}\right)^{(i)} /\left(M_{1} \oplus M_{2}\right)^{(i+1)} \cong M_{1}^{(i)} / M_{1}^{(i+1)} \oplus M_{2}^{(i)} / M_{2}^{(i+1)} .
$$

Assim $M_{1}^{(i)} / M_{1}^{(i+1)}$ e $M_{2}^{(i)} / M_{2}^{(i+1)}$ são $B_{i}$-módulos projetivos, pois são somando diretos de um projetivo, e portanto, pelo Corolário 3.3.4, $M_{1}, M_{2}$ estão em $\mathcal{F}(\triangle)$.

A proposição abaixo, apresentada por Ringel em [24], é uma conseqüência importante de alguns resultados do Capítulo 2 e da Seção 3.2.

Proposição 3.3.6. As categorias $\mathcal{F}(\triangle)$ e $\mathcal{F}(\nabla)$ são funtorialmente finitas e, portanto, admitem seqüências de Auslander-Reiten relativas.

Demonstração. Como $\operatorname{Ext}_{A}^{1}\left(\triangle_{i}, \triangle_{j}\right)=0$, para $j \leq i$, pela Proposição 3.2.8, então $\operatorname{Ext}_{A}^{1}\left(\nabla_{i}, \nabla_{j}\right)=\operatorname{Ext}_{A}^{1}\left(D\left(\triangle_{i}^{o}\right), D\left(\triangle_{j}^{o}\right)\right) \cong D \operatorname{Ext}_{A^{o p}}^{1}\left(\triangle_{j}^{o}, \triangle_{i}^{o}\right)=0$, para $i \leq j$. Assim, pelo Teorema 2.1.11, tanto $\mathcal{F}(\triangle)$ quanto $\mathcal{F}(\nabla)$ são 
funtorialmente finitas em $\bmod A$. Mas como também são fechadas por somandos diretos (Corolário 3.3.5), temos que $\mathcal{F}(\triangle)=\mathcal{X}(\triangle)$ e que $\mathcal{F}(\nabla)=$ $\mathcal{X}(\nabla)$. Logo, pela Proposição 2.1.15, resulta que elas admitem seqüências de Auslander-Reiten relativas.

Antes de apresentar outras propriedades das categorias $\mathcal{F}(\triangle)$ e $\mathcal{F}(\nabla)$, fixaremos algumas notações e faremos algumas observações para usá-las nas demonstrações que seguem.

Seja $M \in \mathcal{F}(\triangle)$. Denotamos por $\left[M: \triangle_{i}\right]$ a multiplicidade de $\triangle_{i}$ em uma $\triangle$-filtração de $M$. De forma análoga, se $M \in \mathcal{F}(\nabla)$, denotamos por $\left[M: \nabla_{i}\right]$ a multiplicidade de $\nabla_{i}$ em uma $\nabla$-filtração de $M$. É importante notar que tais multiplicidades não dependem da escolha da filtração como veremos a seguir.

Proposição 3.3.7. O número $\left[M: \triangle_{i}\right]$ não depende da escolha da $\triangle$ filtração para $M$.

Demonstração. Sejam $D=\left[d_{i j}\right]$, onde $d_{i j}=\operatorname{dim}_{K} \operatorname{Hom}_{A}\left(\triangle_{i}, \triangle_{i}\right)$, e $c_{i}=\operatorname{dim}_{K}\left(\operatorname{Hom}_{R}\left(M, \nabla_{i}\right)\right)$. Como temos que $\operatorname{Hom}_{R}\left(\triangle_{j}, \triangle_{i}\right)=0$, para $j>i$, então $d_{i j}=0$, para $j>i$. Mas tambem $d_{i i}=\operatorname{End}_{A}\left(\triangle_{i}\right) \neq 0$. Portanto a matriz $D$ é triangular superior e $\operatorname{det} D \neq 0$.

Sejam $M \in \mathcal{F}(\triangle)$ e $\xi: 0=M_{0} \subset M_{1} \subset \ldots \subset M_{m-1} \subset M_{m}=M$ uma $\triangle$-filtração para $M$. Então como o funtor $\operatorname{Hom}_{A}\left(\Perp_{i=1}^{n} \nabla_{i}\right)$ é exato em $\mathcal{F}(\triangle)$, temos que

$$
c_{i}=\operatorname{dim}_{K}\left(\operatorname{Hom}_{A}\left(M, \nabla_{i}\right)\right)=\sum_{j=1}^{t} m_{\xi}(j) d_{i j}=\left(m_{\xi}(1), m_{\xi}(2), \ldots m_{\xi}(t)\right) D_{i},
$$


onde $m_{\xi}(j)$ é a multiplicidade de $\left.\triangle_{j}\right)$ na $\triangle$-filtração $\xi$ e $D_{i}$ é a $i$-ésima coluna da matriz $D$. Logo, usando que det $D \neq 0$, obtemos que $m_{\xi}(j)$ depende de $c_{i}$ e de $d_{i j}$, que não dependem de $\xi$.

Definição 3.3.8. Seja $M \in \bmod A . O \triangle$-suporte de $M$, que denotaremos por $\operatorname{Supp}_{\triangle}(M)$, é o conjunto $\operatorname{Supp}_{\triangle}(M)=\left\{i \in\{1,2, \ldots, n\}:\left[M: \triangle_{i}\right] \neq 0\right\}$.

Observação 3.3.9. Sejam $M, N \in \bmod A$ e $f \in \operatorname{Hom}_{A}(M, N)$. Então:

1. Para cada $i, f\left(M^{(i)}\right) \subseteq N^{(i)}$. Mais ainda, se $f$ é um epimorfismo, então $f\left(M^{(i)}\right)=N^{(i)}$. Para a primeira afirmação note-se que para toda $h \in \operatorname{Hom}_{A}\left(\varepsilon_{i} A, M\right)$ temos que $f h \in \operatorname{Hom}_{A}\left(\varepsilon_{i} A, N\right)$ e que $f(\operatorname{Im} h)=$ Im $f h$. Para a segunda, se $f$ é epimorfismo, para toda $t \in \operatorname{Hom}_{A}\left(\varepsilon_{i} A, N\right)$, exite $g \in \operatorname{Hom}_{A}\left(\varepsilon_{i} A, M\right)$ tal que $f h=t$ e, portanto, $f\left(g\left(\varepsilon_{i} A\right)\right)=$ $t\left(\varepsilon_{i} A\right)$. Isto ultimo implica que $N^{(i)} \subset f\left(M^{(i)}\right)$.

2. Se $M, N \in \mathcal{F}(\triangle)$ e $f$ é um epimorfismo, então

$$
\operatorname{Supp}_{\triangle}(N) \subseteq \operatorname{Supp}_{\triangle}(M) .
$$

De fato, seja $i \in S u p p \triangle(N)$. Então, pela Proposição 3.3.2 e pelo observado em (1), existe um inteiro $t_{i}>0$ tal que

$$
\triangle_{i}^{t_{i}} \cong N^{(i)} / N^{(i+1)}=f\left(M^{(i)}\right) / f\left(M^{(i+1)}\right) .
$$

Isto quer dizer que o quociente $f\left(M^{(i)}\right) / f\left(M^{(i+1)}\right) \neq 0$ ou equivalentemente que $f\left(M^{(i)}\right) \neq 0$ e $f\left(M^{(i+1)}\right) \subsetneq f\left(M^{(i)}\right)$. Segue daí que $M^{(i)} \neq 0$ e $M^{(i+1)} \subsetneq M^{(i)}$. Assim $M^{(i)} / M^{(i+1)} \neq 0$ e, portanto, $i \in \operatorname{Supp}_{\triangle}(M)$.

A demonstração da seguinte proposição seguinte é feita como em [26]. 
Proposição 3.3.10. A categoria $\mathcal{F}(\triangle)$ é fechada por núcleos de epimorfismos.

Demonstração. Sejam $M, N \in \mathcal{F}(\triangle)$ e $f: M \longrightarrow N$ um epimorfismo de $A$-módulos. Vamos provar, por indução sobre a cardinalidade de $\operatorname{Supp}_{\triangle}(M)$, que $L=\operatorname{ker}(f) \in \mathcal{F}(\triangle)$.

Se $\left|\operatorname{Supp}_{\triangle}(M)\right|=1$, então pela parte (2) da observação acima, temos que $\left|\operatorname{Supp}_{\triangle}(N)\right|=1$. Logo, pela Proposição 3.3.2, $M \cong \triangle_{i}^{s}$ e $N \cong \triangle_{i}^{t}$, com $s>t$. Agora, aplicando o funtor $\operatorname{Hom}_{A}(\ldots, L)$ na seqüência exata $0 \longrightarrow U_{i} \longrightarrow P_{i} \longrightarrow \triangle_{i} \longrightarrow 0$ obtemos a seqüência exata

$0 \longrightarrow \operatorname{Hom}_{A}\left(\triangle_{i}, L\right) \longrightarrow \operatorname{Hom}_{A}\left(P_{i}, L\right) \longrightarrow \operatorname{Hom}_{A}\left(U_{i}, L\right) \longrightarrow \operatorname{Ext}_{A}^{1}\left(\triangle_{i}, L\right) \longrightarrow 0$

Afirmamos que com $\operatorname{Hom}_{A}\left(U_{i}, L\right)=0$. De fato, suponhamos que $\operatorname{Hom}_{A}\left(U_{i}, L\right) \neq 0$. Seja $h \in \operatorname{Hom}_{A}\left(U_{i}, L\right)$, com $h \neq 0$. Portanto, existem um $x \in U_{i}$ e um homomorfismo $g: P_{j} \rightarrow P_{i}$, com $j>i$, tais que $x \in \operatorname{Img}$ e $h(x) \neq 0$. Assim a composta $h g^{\prime}: P_{j} \longrightarrow L$, onde $g^{\prime}=i g$, onde $i: U_{i} \longrightarrow P_{i}$ é a inclusão canônica, é não nula.

Logo, tanto $L$ quanto $M$ têm um fator de composição isomorfo a $S_{j}$, para um $j>i$, o que é uma contradição. De $\operatorname{Hom}_{A}\left(U_{i}, L\right)=0$ resulta que $\operatorname{Ext}_{A}^{1}\left(\triangle_{i}, L\right)=0$, seguindo daí que a sequiência $0 \longrightarrow L \longrightarrow M \longrightarrow N \longrightarrow 0$ cinde e que $L \cong \triangle_{i}^{s-t}$. E está provada a afirmação quando $\left|\operatorname{Supp}_{\triangle}(M)\right|=1$.

Seja $M \in \bmod A$ tal que $\left|\operatorname{Supp}_{\triangle}(M)\right|=\ell \geq 2$. Suponhamos que se existe um epimorfismo $f: M^{\prime} \longrightarrow N^{\prime} \operatorname{com} M^{\prime}, N^{\prime} \in \mathcal{F}(\triangle)$ e $\left|S_{u p p} \triangle\left(M^{\prime}\right)\right|<\ell$, então $\operatorname{Ker}(f) \in \mathcal{F}(\triangle)$. Mostremos que a conclusão também vale para $M$. Seja $t=\max \operatorname{Supp}_{\triangle}(M)$. Pela Observação 3.3.3, os módulos $M^{(t)}$ e $N^{(t)}$ são tais que $M / M^{(t)}, N / N^{(t)} \in \mathcal{F}\left(\left\{\triangle_{1}, \triangle_{2}, \ldots \triangle_{t-1}\right\}\right)$ e $M^{(t)}, N^{(t)} \in \operatorname{add}\left(\triangle_{t}\right)$. 
Agora consideremos o diagrama

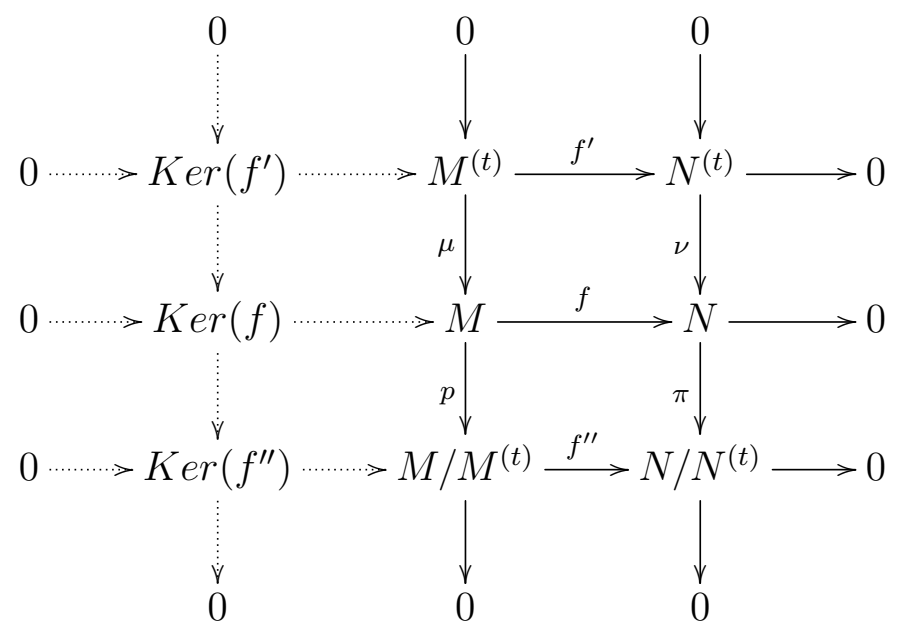

onde $f^{\prime}=\left.f\right|_{M^{(t)}}$ e $f^{\prime \prime}: M / M^{(t)} \longrightarrow N / N^{(t)}$ é tal que $f^{\prime \prime}\left(m+M^{(t)}\right)=f(m)+$ $N^{(t)}$. Tanto $f^{\prime}$ quanto $f^{\prime \prime}$ são epimorfismos, pois $f^{\prime}\left(M^{(t)}\right)=f\left(M^{(t)}\right)=N^{(t)}$. O diagrama de linhas continuas é comutativo já que $\mu$ e $\nu$ são inclusões e $p$ e $\pi$ são as projeções canônicas. Assim o Lema da Serpente garante a existência da seqüencia exata

$$
0 \longrightarrow \operatorname{ker}\left(f^{\prime}\right) \longrightarrow \operatorname{ker}(f) \longrightarrow \operatorname{Ker}\left(f^{\prime \prime}\right) \longrightarrow 0 \text {. }
$$

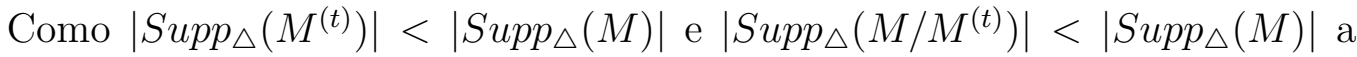
hipótese de indução implica que tanto $\operatorname{ker}\left(f^{\prime}\right)$ quanto $k e r\left(f^{\prime \prime}\right)$ estão em $\mathcal{F}(\triangle)$. Isto prova que ker $f \in \mathcal{F}(\triangle)$, pois $\mathcal{F}(\triangle)$ é fechada por extensões (Observação 2.1.2).

Dualmente temos o seguinte resultado para a categoria $\mathcal{F}(\nabla)$.

Proposição 3.3.11. A categoria $\mathcal{F}(\nabla)$ é fechada por conúcleos de monomorfismos. 


\section{4 Álgebras estandarmente estratificadas}

Nesta seção introduziremos os conceitos de álgebra estandarmente estratificada e álgebra quase-hereditária. Demonstraremos que para as álgebras estandarmente estratificada os módulos em $\mathcal{F}(\triangle)$ têm dimensão projetiva finita. Provaremos também que as álgebras quase-hereditárias tem dimensão global finita. Os resultados contidos aqui estão em [15].

Primeiramente definiremos álgebras estandarmente estratificadas.

Definição 3.4.1. Sejam A uma K-álgebra, $\triangle e \nabla$ as seqüências dos módulos estandares e dos módulos coestandares, respectivamente, relativas a uma ordem $\boldsymbol{e}=\left(e_{1}, e_{2}, \ldots, e_{n}\right)$ fixada dos idempotentes. Dizemos que A é uma álgebra estandarmente estratificada se $A_{A} \in \mathcal{F}(\triangle)$. Dizemos que $A$ é uma álgebra coestandarmente estratificada se $D A \in \mathcal{F}(\nabla)$.

Uma caracterizacão das álgebras estandarmente estratificadas e das coestandarmente estratificadas está expressa na proposição abaixo.

Proposição 3.4.2. Uma $K$-álgebra $A$ é estandarmente estratificada se, e somente se, todos os A-módulos projetivos indecomponíveis são $\triangle$-filtrados. De forma dual, A é coestadarmente estratificada se, e somente se, todos os A-módulos injetivos indecomponíveis estão em $\mathcal{F}(\nabla)$.

Demonstração. $\quad$ Seja $A=e_{1} A \oplus e_{2} A \oplus \ldots \oplus e_{n} A$ uma decomposição de $A$ como $A$-módulo à direita, em módulos indecomponíveis.

Suponhamos que $A_{A} \in \mathcal{F}(\triangle)$. Então, para cada $i=1,2, \ldots, n$, o $A$ módulo projetivo $P_{i}=e_{i} A \in \mathcal{F}(\triangle)$, pois $\mathcal{F}(\triangle)$ é fechada por somandos 
diretos. Reciprocamente, suponhamos que todos os $A$-módulos projetivos indecomponíveis estejam em $\mathcal{F}(\triangle)$. Consideremos a seqüência $0 \rightarrow P_{1} \rightarrow$ $P_{1} \oplus P_{2} \rightarrow P_{2} \rightarrow 0$. Temos que $P_{1} \oplus P_{2} \in \mathcal{F}(\triangle)$, pois é $\mathcal{F}(\triangle)$ é fechada por extensões. Da mesma forma, considerando a seqüência $0 \rightarrow P_{3} \rightarrow P_{1} \oplus$ $P_{2} \oplus P_{3} \rightarrow P_{1} \oplus P_{2} \rightarrow 0$, concluímos que $P_{1} \oplus P_{2} \oplus P_{3} \in \mathcal{F}(\triangle)$. Repetindo o processo, podemos concluir que $A_{A} \in \mathcal{F}(\triangle)$. A demonstração da afirmação dual é feita de forma análoga.

O exemplo a seguir mostra que a propriedade de uma álgebra ser estandarmente estratificada depende da ordem fixada para os idempotentes.

Exemplo 3.4.3. Seja $A \cong k Q / I$ onde $I=\left\langle\alpha \beta, \beta^{2}\right\rangle$ e $Q$ é o carcás

$$
1 \cdot \stackrel{\alpha}{\longrightarrow} \cdot 2 \bigcirc \beta
$$

Na ordem $\boldsymbol{e}_{\mathbf{1}}=\left(e_{1}, e_{2}\right)$, temos $\triangle=\left\{\triangle_{1}=S_{1}, \triangle_{2}=P_{2}\right\}$, e que $A$ não é estandarmente estratificada, pois a única cadeia de submódulos de $P_{1}$ é $0 \subset$ $\operatorname{rad} P_{1}=S_{2} \subset P_{1}$, que não é uma $\triangle$-filtração uma vez que $S_{2} / 0 \cong S_{2} \notin \triangle$. No entanto, na ordem $\boldsymbol{e}_{\mathbf{2}}=\left(e_{2}, e_{1}\right)$, temos que $\triangle=\left\{\triangle_{1}=P_{2}, \triangle_{2}=P_{1}\right\}$, e que $A \in \mathcal{F}(\triangle)$, o que implica que $A$ é estandarmente estratificada.

F. Advincula e E. Marcos caracterizaram em [18] as álgebras que são estandarmente estratificadas para uma ordenação qualquer de um conjunto de idempotentes primitivos e ortogonais: elas são exatamente aquelas cujos ideais idempotentes são módulos projetivos.

Um outro problema interessante e mais difícil é caracterizar aquelas álgebras que são estandarmente estratificadas numa única ordem. 
Vamos introduzir agora as definições de subcategoria resolvente e corresolvente em $\bmod A$.

Definição 3.4.4. Dizemos que uma subcategoria $\mathcal{X}$ de $\bmod A$ é resolvente quando é fechada por extensões, por núcleos de epimorfismos e contém todos os projetivos. Dualmente, uma subcategoria $\mathcal{X}$ de $\bmod A$ é corresolvente quando é fechada por extensões, por conúcleos de monomorfismos e contém todos os injetivos.

Com a definição acima, as Proposições 3.3.10, 3.3.11 e 3.4.2 e mais o fato de que $\mathcal{F}(\triangle)$ e $\mathcal{F}(\nabla)$ são subcategorias fechadas por extensões (Observação 2.1.2), obtemos o seguinte corolário.

Corolário 3.4.5. Sejam A uma K-álgebra, $\triangle e \nabla$ as seqüências de módulos estandares e coestandares, relativas a uma ordem e. Então:

1. Se $A_{A} \in \mathcal{F}(\triangle)$, então $\mathcal{F}(\triangle)$ é uma categoria resolvente em $\bmod A$.

2. Se $D A \in \mathcal{F}(\nabla)$, então $\mathcal{F}(\nabla)$ é uma categoria coresolvente em $\bmod A$.

Introduziremos agora o conceito de seqüência estandar Schurian, que é utilizada para definir ás álgebras quase-hereditárias.

Definição 3.4.6. Dizemos que a seqüência $\triangle=\left(\triangle_{1}, \triangle_{2}, \ldots, \triangle_{n}\right)$ de módulos estandares é Schurian se cada $\triangle_{i}$ é Schurian ; isto é, se $\operatorname{End}_{A}\left(\triangle_{i}\right)$ é um anel com divisão para $i=1,2, \ldots, n$. De forma analoga, dizemos que a seqüência $\nabla=\left(\nabla_{1}, \nabla_{2}, \ldots, \nabla_{n}\right)$ de módulos coestandares é Schurian se $\operatorname{End}_{A}\left(\nabla_{i}\right)$ é um anel com divisão para $i=1,2, \ldots, n$. 
Como temos trabalhado sempre no caso em que o corpo $K$ é algebricamente fechado, então dizer que $\triangle_{i}$ é Schurian é equivalente a dizer que $\operatorname{End}_{A}\left(\triangle_{i}\right) \cong K$.

A proposição seguinte fornece outras reformulações da definição acima.

Proposição 3.4.7. Para $i=1,2, \ldots, n$, as seguintes condições são equivalentes:

1. $\triangle_{i}$ é Schurian.

2. $\operatorname{End}_{A}\left(\triangle_{i}\right) \cong K$.

3. $e_{i} A \varepsilon_{i+1} A e_{i}=e_{i}(\operatorname{rad} A) e_{i}$.

4. $\left[\triangle_{i}: S_{i}\right]=1$.

Demonstração. Pela observação feita na definição acima, temos que (1) é equivalente a (2). Observemos agora que da aplicação do funtor $\operatorname{Hom}_{A}\left(\ldots, \triangle_{i}\right)$ à sequiência exata $0 \longrightarrow U_{i} \longrightarrow P_{i} \longrightarrow \triangle_{i} \longrightarrow 0$, e da Proposição 3.2 .8 (2),

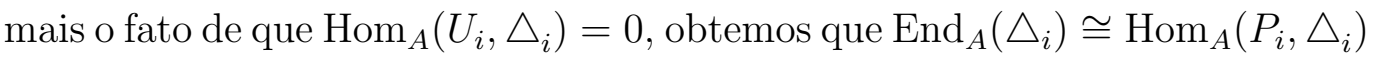
e, por outro lado,

$$
\operatorname{Hom}_{A}\left(P_{i}, \triangle_{i}\right)=\operatorname{Hom}_{A}\left(e_{i} A, e_{i} A / e_{i} A \varepsilon_{i+1} A e_{i}\right) \cong\left(e_{i} A / e_{i} A \varepsilon_{i+1} A e_{i}\right) e_{i}
$$

portanto, temos que

$$
\operatorname{rad}\left(\operatorname{End}_{A}\left(\triangle_{i}\right)\right) \cong \frac{\operatorname{rad}\left(e_{i} A e_{i}\right)+e_{i} A \varepsilon_{i+1} A e_{i}}{e_{i} A \varepsilon_{i+1} A e_{i}} .
$$

Isto significa que $\operatorname{End}_{A}\left(\triangle_{i}\right) \cong K$ se, e somente se, $e_{i} A \varepsilon_{i+1} A e_{i}=e_{i}(\operatorname{rad} A) e_{i}$. Logo (2) equivale a (3). 
Para concluir mostraremos que (2) equivale a (4), o que é conseqüência de que $\left[\triangle_{i}: S_{i}\right]=\operatorname{dim}_{K} \operatorname{Hom}_{A}\left(P_{i}, \triangle_{i}\right)$ e de que $\operatorname{Hom}_{A}\left(P_{i}, \triangle_{i}\right) \cong \operatorname{End}_{A}\left(\triangle_{i}\right)$, como foi visto acima.

Dualmente temos a seguinte proposição.

Proposição 3.4.8. As seguintes condições são equivalentes:

1. $\triangle_{i}^{o}\left(\right.$ e portanto $\left.\nabla_{i}\right)$ é Schurian.

2. $\operatorname{End}_{A}\left(\nabla_{i}\right) \cong K$.

3. $\left[\triangle_{i}^{o}: S_{i}\right]=\left[\nabla_{i}: S_{i}\right]=1$.

Obsevemos que, dada uma ordem $\boldsymbol{e}$ se $\triangle$ é Schurian, então $\triangle_{1} \cong \nabla_{1} \cong S_{1}$.

Definição 3.4.9. Dizemos $A$ é uma álgebra quase-hereditária se $A$ é uma álgebra estandarmente estratificada e a seqüência $\triangle$ é Schurian.

Mostraremos agora um exemplo de uma álgebra que numa dada ordem $\boldsymbol{e}_{1}$ é quase-hereditária (e portanto estandarmente estratificada) e que numa ordem $\boldsymbol{e}_{2}$ não é estandarmente estratificada (e portanto não é quasehereditária).

Exemplo 3.4.10. Sejam $K$ um corpo algebricamente fechado e $A=K Q / I$, onde $Q$ é o carcás

$$
i \stackrel{\alpha_{1}}{\longrightarrow}{ }_{2} \stackrel{\alpha_{2}}{\longrightarrow} \underset{3}{\longrightarrow} \cdots \longrightarrow \underset{n-1}{\stackrel{\alpha_{n-1}}{\longrightarrow}}{ }_{n}
$$


e $I=\left\langle\alpha_{i} \alpha_{i+1}: i=1, \ldots, n-2\right\rangle$.

Os $A$-módulos projetivos indecomponíveis são os módulos associados às representações

$$
\begin{aligned}
P_{n}: & { }_{0} \longrightarrow{ }_{0} \longrightarrow{ }_{0} \longrightarrow \cdots{ }_{0} \longrightarrow{ }_{0} \longrightarrow{ }_{K} \\
P_{i}: & { }_{0} \longrightarrow{ }_{0}{ }_{0} \longrightarrow{ }_{K}{ }^{i} \stackrel{i d_{K}}{\longrightarrow}{ }_{K}^{i+1} \longrightarrow{ }_{0} \cdots_{0} \longrightarrow{ }_{0}, \quad \text { para } 1 \leq i \leq n-1 .
\end{aligned}
$$

Logo para a ordem $\boldsymbol{e}_{\mathbf{1}}=(1,2, \ldots, n)$ temos que $\triangle_{i}=S_{i}$ e, portanto, $A$ é quase-hereditária.

De outro lado, na ordem $\boldsymbol{e}_{2}=(n, 1,2, \ldots, n-1)$ temos que $\triangle_{n}=S_{i}$, para $1 \leq i \leq n-2$, e que $\triangle_{i}=P_{i}$, para $i=n-1, n$. Como uma série de composição para $P_{n-2}$ é $0 \subset \operatorname{rad} P_{n-2}=S_{n-1} \subset P_{n-2}$ e $S_{n-1} \notin \triangle$, nesta ordem $A$ não é estandarmente estratificada e, portanto, não é quase-hereditária.

Com o objetivo de estudar as dimensões homológicas dos módulos em $\mathcal{F}(\triangle)$, no caso em que $A$ é uma álgebra estandarmente estratificada, vamos enunciar o seguinte lema que é bem conhecido e cuja demonstração pode ser encontrada em [3].

Lema 3.4.11. Seja $0 \longrightarrow X \longrightarrow Y \longrightarrow Z \longrightarrow 0$ uma seqüência exata em $\bmod A$. Então:

1. $p d Y \leq \max \{p d X, p d Z\}$.

2. $p d Z \leq \max \{p d Y, 1+p d X\}$. 
Corolário 3.4.12. Sejam $X, M_{1}, M_{2}, \ldots, M_{t}$ uma família de A-módulos de comprimento finito. Se $X \in \mathcal{F}\left(\left\{M_{1}, M_{2}, \ldots, M_{t}\right\}\right)$. Então

$$
p d X \leq \max _{1 \leq i \leq t}\left\{p d M_{i}\right\} .
$$

Demonstração. Por indução sobre o comprimento de $X$. Se $\ell(X)=1$, então $X=M_{i}$, para algum $j, 1 \leq j \leq t$, e a afirmação é verdadeira.

Suponhamos que a afirmação é valida para os $A$-módulos de comprimento menor do que $m, m \geq 2$. Seja $X \in \mathcal{F}\left(\left\{M_{1}, M_{2}, \ldots, M_{t}\right\}\right)$ tal que $\ell(X)=m$. Então existe um submódulo próprio $Y$ de $X$ tal que $Y \in$ $\mathcal{F}\left(\left\{M_{1}, M_{2}, \ldots, M_{t}\right\}\right)$ e que $X / Y \cong M_{i}$, para algum $i, 1 \leq i \leq t$.

Considerando a seqüência exata $0 \longrightarrow Y \longrightarrow X \longrightarrow M_{i} \longrightarrow 0$, temos que $p d X \leq \max \left\{p d M_{i}, p d Y\right\}$, Mas como $\ell(Y)<m$, pela hipótese de indução segue que $p d Y \leq \max _{1 \leq i \leq t}\left\{p d M_{i}\right\}$ e portanto $p d X \leq \max _{1 \leq i \leq t}\left\{p d M_{i}\right\}$.

Proposição 3.4.13. Seja A uma K-álgebra quase-hereditária. Então, para cada $i=1, \ldots, n$, vale que :

1. $p d \triangle_{i} \leq n-i$.

2. $p d S_{i} \leq n+i-2$.

3. $\operatorname{gldim} A \leq 2(n-1)$.

Demonstração. Como $A$ é estandarmente estratificada, as Proposições 3.4 .2 e 3.3.10, garantem que a seqüência exata $0 \longrightarrow U_{i} \longrightarrow P_{i} \longrightarrow \triangle_{i} \longrightarrow 0$ está em $\mathcal{F}(\triangle)$. De outro lado, pelo Lema 3.4.11 (2), temos $p d \triangle_{i} \leq 1+p d U_{i}$. Além disso, de acordo com a Observação 3.3.3, $U_{i}=P_{i}^{(i+1)}$ é filtrado por $\triangle_{j}$, 
com $j>i$, logo, pelo Corolário 3.4.12, resulta que $p d U_{i} \leq \max _{j>i}\left\{p d \triangle_{j}\right\}$. Assim $p d \triangle_{i} \leq 1+\max _{j>i}\left\{p d \triangle_{j}\right\}$.

Para provar (1) faremos indução regressiva sobre $i$. Para $i=n, \triangle_{n}=P_{n}$ e portanto $p d \triangle_{n}=0$. Vamos supor que a desigualdade é verdadeira para todos os inteiros $t$ tais que $m \leq t \leq n$, com $m>0$. Temos pois que

$$
\begin{aligned}
p d \triangle_{m-1} & \leq 1+\max _{j>m-1}\left\{p d \triangle_{j}\right\} \\
& \leq 1+\max \{0, n-(n-1), n-(n-2), \ldots, n-m\} \\
& =n-(m-1),
\end{aligned}
$$

com o que (1) está provado.

Para provar (2) consideremos a seqüência exata

$$
0 \longrightarrow V_{i} \longrightarrow \triangle_{i} \longrightarrow S_{i} \longrightarrow 0 \text {, onde } V_{i}=\operatorname{rad} \triangle_{i} .
$$

Do Lema 3.4.12 resulta que $p d S_{i} \leq 1+\max \left\{p d V_{i}, p d \triangle_{i}\right\}$. De outro lado, pelo Lema 3.2.5, os fatores de composição de $\triangle_{i}$ são $S_{j} \operatorname{com} j \leq i$. Como $\triangle_{i}$ é Schurian, pela Proposição 3.4.7, $\left[\triangle_{i}: S_{i}\right]=1 \mathrm{e}$, em conseqüência os fatores de composição de $V_{i}$ são $S_{j} \operatorname{com} j<i$. Portanto, $p d S_{i} \leq 1+\max _{j<i}\left\{p d S_{j}, p d \triangle_{i}\right\}$.

Continuamos a prova usando uma indução ascendente sobre $i$. Para $i=1$, $\triangle_{1}=S_{1}$ e por (1) vale que $p d \triangle_{1} \leq n-1=(n+1)-2$, e a afirmação (2) está verificada nesse caso.

Suponhamos que a desigualdade vale para os inteiros $t$ tais que $0<t \leq m$, com $2 \leq m \leq n$. Então $p d S_{m+1} \leq 1+\max _{t<m+1}\left\{p d S_{t}, p d \triangle_{m+1}\right\}$. Por (1) e pela hipótese de indução, temos que

$$
p d S_{m+1} \leq 1+\max _{t<m+1}\{n+t-2, n-(m+1)\} \leq 1+n+(m-2)
$$

e (2) está provado.

Por fim, usando (2), temos gldim $A=\max _{1 \leq i \leq n}\left\{p d S_{i}\right\} \leq 2 n-2$ e (3) está provado. 
Observemos que na demonstração da primeira parte da Proposição 3.4.13 só usamos o fato de que $A$ é uma álgebra estandarmente estratificada. Assim temos a seguinte conseqüência imediata.

Corolário 3.4.14. Sejam A uma álgebra estandarmente estratificada e $X \in$ $\mathcal{F}(\triangle)$. Então pd $X \leq n-1$.

Com a finalidade de mostrar que este limitante superior para a dimensão global é o melhor possível consideraremos o seguinte exemplo, que foi dado em [15] por Dlab.

Exemplo 3.4.15. Sejam $K$ um corpo algebricamente fechado e $A=K Q / I$, onde $Q$ é o carcás

$$
\cdot 1 \frac{\alpha_{(1,2)}}{\underset{\alpha_{(2,1)}}{\longrightarrow}} \cdot 2 \frac{\alpha_{(2,3)}}{\underset{\alpha_{(3,2)}}{\longrightarrow}} \cdot 3 \frac{\alpha_{(3,4)}}{\underset{\alpha_{(4,3)}}{\longrightarrow}} \cdot 4
$$

e $I=\left\langle\alpha_{(4,3)} \alpha_{(3,2)}, \alpha_{(3,2)} \alpha_{(2,1)}, \alpha_{(1,2)} \alpha_{(2,3)}, \alpha_{(2,3)} \alpha_{(3,4)}, \alpha_{(2,1)} \alpha_{(1,2)}, \alpha_{(3,2)} \alpha_{(2,3)}, \alpha_{(4,3)} \alpha_{(3,4)}\right\rangle$.

Os $A$-módulos projetivos indecomponíveis são os módulos associados às seguintes representações:

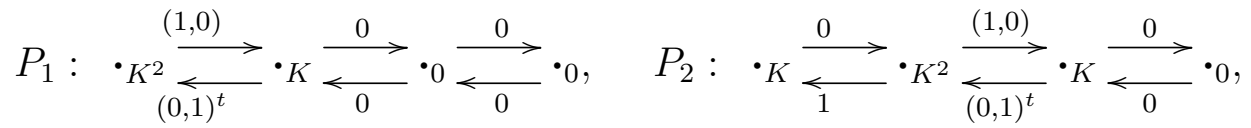

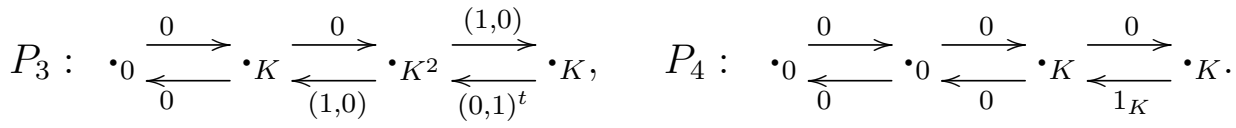

A álgebra $A$ é quase-hereditária, pois $\triangle_{1}=S_{1}, \triangle_{4}=P_{4}$ e $\triangle_{2}$ e $\triangle_{3}$ são os 
módulos associados às representações

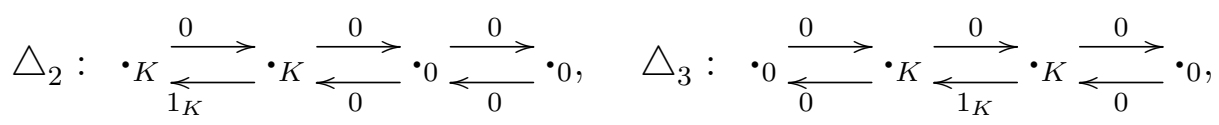

e claramente $\operatorname{End}\left(\triangle_{i}\right) \cong K$, para $1 \leq i \leq 4$. Desde que, as seqüências exatas

$$
\begin{aligned}
& 0 \rightarrow P_{4} \rightarrow P_{3} \rightarrow P_{2} \rightarrow P_{1} \rightarrow S_{1} \rightarrow 0, \\
& 0 \rightarrow P_{4} \rightarrow P_{3} \rightarrow P_{2} \oplus P_{4} \longrightarrow P_{1} \oplus P_{3} \rightarrow P_{2} \rightarrow S_{2} \rightarrow 0, \\
& 0 \rightarrow P_{4} \rightarrow P_{3} \rightarrow P_{2} \oplus P_{4} \rightarrow P_{1} \oplus P_{3} \rightarrow P_{2} \oplus P_{4} \rightarrow P_{3} \rightarrow S_{3} \rightarrow 0, \\
& 0 \rightarrow P_{4} \rightarrow P_{3} \rightarrow P_{2} \oplus P_{4} \rightarrow P_{1} \oplus P_{3} \rightarrow P_{2} \oplus P_{4} \rightarrow P_{3} \rightarrow P_{4} \rightarrow S_{4} \rightarrow 0
\end{aligned}
$$

são resoluções projetivas minimais temos que gldim $A=6=2(4-1)$.

Nesta seção também estudaremos algumas novas propriedades das categorias $\mathcal{F}(\triangle)$ e $\mathcal{F}(\nabla)$ no caso em que $A$ é uma álgebra estandarmente estratificada.

Para isso, vamos relembrar algumas categorias relacionadas com $\mathcal{F}(\triangle)$ e $\mathcal{F}(\nabla)$, cujas notações e propriedades foram exibidas no Capítulo 2, para $\mathcal{F}(\theta)$.

Nesse caso, lembramos que $\mathcal{Y}(\triangle)$ denota a subcategoria plena de $\bmod A$ formada pelos objetos $Y$ tais que $\operatorname{Ext}_{A}^{1}\left(\triangle_{j}, Y\right)=0$, para $j=1, \ldots, n$. Mais ainda que $\mathcal{W}(\nabla)$ indica a subcategoria plena de $\bmod A$ cujos objetos $W$ são tais que $\operatorname{Ext}_{A}^{1}\left(W, \nabla_{j}\right)=0$, para $j=1, \ldots, n$.

Recordamos também a noção de módulos projetivos e injetivos relativos. Sejam $\mathcal{C}$ uma subcategoria de $\bmod A$ e $X$ um $A$-módulo em $\mathcal{C}$. Dizemos que $X$ é Ext-projetivo em $\mathcal{C}$ se $\operatorname{Ext}_{A}^{1}(X, Y)=0$, para todo $Y$ em $\mathcal{C}$. De forma dual, dizemos que $X$ é Ext-injetivo em $\mathcal{C}$ se $\operatorname{Ext}_{A}^{1}(Y, X)=0$, para todo $Y$ em $\mathcal{C}$.

Segundo as definições relembradas acima, os módulos relativamente inje- 
tivos em $\mathcal{F}(\triangle)$ são os elementos do conjunto $\mathcal{Y}(\triangle) \cap \mathcal{F}(\triangle)$ e os módulos relativamente projetivos em $\mathcal{F}(\nabla)$ são os elementos do conjunto $\mathcal{W}(\nabla) \cap \mathcal{F}(\nabla)$.

Proposição 3.4.16. Seja A uma K-álgebra estandarmente estratificada. Então os módulos Ext-projetivos de $\mathcal{F}(\triangle)$ são os A-módulos projetivos.

Demonstração. Como $A$ é estandarmente estratificada, então cada $A$ módulo projetivo está em $\mathcal{F}(\triangle)$ e por isso é Ext-projetivo em $\mathcal{F}(\triangle)$.

Seja $M$ um $A$-módulo Ext-projetivo em $\mathcal{F}(\triangle)$. Para mostrar que $M$ é projetivo consideremos uma a seqüência exata qualquer, em $\bmod A$, que termina em $M$

$$
0 \longrightarrow X \stackrel{g}{\longrightarrow} L \stackrel{f}{\longrightarrow} M \longrightarrow 0
$$

Seja $\gamma: X \longrightarrow X_{1}$ uma $\mathcal{F}(\triangle)$-aproximação à direita de $X$, que existe pois $\mathcal{F}(\triangle)$ é funtorialmente finita, conforme a Proposição 3.3.6. Assim obtemos o seguinte diagrama comutativo

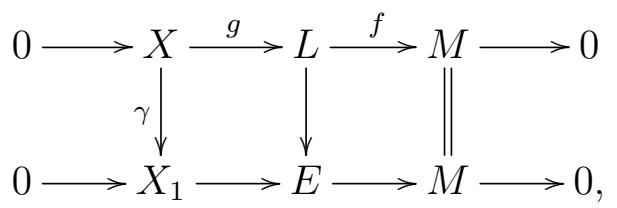

onde

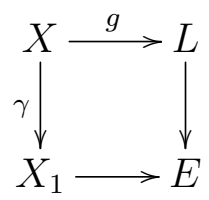

é o push-out de $X_{1} \leftarrow X \rightarrow L$. Como $X_{1} \in \mathcal{F}(\triangle)$ e esta é uma categoria fechada por extensões, temos que a seqüência exata $0 \longrightarrow X_{1} \longrightarrow E \longrightarrow M \longrightarrow 0$ cinde, e portanto a sequência (3.3) também cinde. Portanto o módulo $M$ é projetivo. 
Proposição 3.4.17. Seja A uma álgebra estandarmente estratificada. Então, para cada $i \geq 1, \operatorname{Ext}_{A}^{i}(X, Y)=0, \forall X \in \mathcal{F}(\triangle), \forall Y \in \mathcal{Y}(\triangle)$.

Demonstração. Seja $X \in \mathcal{F}(\triangle)$. Por definição $\operatorname{Ext}_{A}^{1}(X, Y)=0$, para todo $Y \in \mathcal{Y}(\triangle)$. Suponhamos que, para todo $j \geq 2$, os $\operatorname{Ext}_{A}^{j-1}\left(X_{1}, Y\right)=$ $0, \forall X_{1} \in \mathcal{F}(\triangle)$ e $\forall Y \in \mathcal{Y}(\triangle)$. Consideremos então a seqüência exata $0 \longrightarrow X_{1} \rightarrow P \stackrel{\gamma}{\longrightarrow} X \longrightarrow 0$, onde $\gamma: P \longrightarrow X$ é a cobertura projetiva de $X$. Como $A$ é estandarmente estratificada, pelo Corolário 3.4.5, temos que $\mathcal{F}(\triangle)$ é resolvente e, por isso, a seqüência acima está em $\mathcal{F}(\triangle)$, ou seja $X_{1} \in \mathcal{F}(\triangle)$. Portanto, $\operatorname{Ext}_{A}^{j-1}\left(X_{1}, Y\right)=0, \forall Y \in \mathcal{Y}(\triangle)$ pela hipótese de indução. De outro lado, aplicando o funtor $\operatorname{Hom}_{A}(\ldots, Y)$, com $Y \in \mathcal{Y}(\triangle)$, à referida seqüência obtemos a seqüência exata longa

$$
\cdots \rightarrow \operatorname{Ext}_{A}^{1}(P, Y) \rightarrow \operatorname{Ext}_{A}^{1}\left(X_{1}, Y\right) \rightarrow \operatorname{Ext}_{A}^{2}(X, Y) \rightarrow \operatorname{Ext}_{A}^{2}(P, Y) \rightarrow \cdots
$$

Como $P$ é projetivo, então $\operatorname{Ext}_{A}^{j-1}\left(X_{1}, Y\right) \cong \operatorname{Ext}_{A}^{j}(X, Y)$, para todo $j \geq 1$. Logo $\operatorname{Ext}_{A}^{j}(X, Y)=0, \forall j \geq 1$.

Proposição 3.4.18. 1. Seja A uma K-álgebra estandarmente estratificada. Então $\mathcal{Y}(\triangle)$ é uma subcategoria corresolvente de mod $A$.

2. $\mathcal{F}(\nabla) \subseteq \mathcal{Y}(\triangle)$.

Demonstração. Claramente, pela definição, a categoria $\mathcal{Y}(\triangle)$ contém todos os $A$-módulos injetivos. Mostremos $\mathcal{Y}(\triangle)$ é fechada por extensões. Para isso, seja $0 \longrightarrow Y_{1} \longrightarrow Y \longrightarrow Y_{2} \longrightarrow 0$ uma seqüência exata com $Y_{1}$ e $Y_{2}$ em $\mathcal{Y}(\triangle)$. Se aplicamos o funtor $\operatorname{Hom}_{A}\left(X, \_\right), \operatorname{com} X \in \mathcal{F}(\triangle)$, a ela, obtemos 
a seqüência exata longa

$$
\cdots \longrightarrow \operatorname{Ext}_{A}^{1}\left(X, Y_{1}\right) \longrightarrow \operatorname{Ext}_{A}^{1}(X, Y) \longrightarrow \operatorname{Ext}_{A}^{1}\left(X, Y_{2}\right) \longrightarrow \cdots
$$

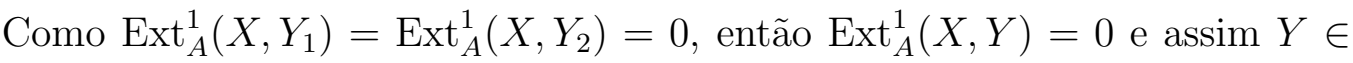
$\mathcal{Y}(\triangle)$, ou seja $\mathcal{Y}(\triangle)$ é realmente fechada por extensões.

Para finalizar, resta provar que $\mathcal{Y}(\triangle)$ é fechada por conúcleos de monomorfismos. Seja então $f: N \longrightarrow M$ um monomorfismo, com $M$ e $N$ em $\mathcal{Y}(\triangle)$. Aplicando $\operatorname{Hom}_{A}\left(\triangle_{i},-\right.$ ), a seqüencia exata $0 \longrightarrow M \longrightarrow N \longrightarrow C \longrightarrow 0$, onde $C$ é o conúcleo de $f$, obtemos seqüencia exata longa

$$
\cdots \longrightarrow \operatorname{Ext}_{A}^{1}\left(\triangle_{i}, N\right) \longrightarrow \operatorname{Ext}_{A}^{1}\left(\triangle_{i}, C\right) \longrightarrow \operatorname{Ext}_{A}^{2}\left(\triangle_{i}, M\right) \longrightarrow \cdots
$$

Mas, pela proposição 3.4.17 temos que $\operatorname{Ext}_{A}^{1}\left(\triangle_{i}, N\right)=0=\operatorname{Ext}_{A}^{2}\left(\triangle_{i}, M\right)$. Portanto $\operatorname{Ext}_{A}^{1}\left(\triangle_{i}, C\right)=0$, para cada $1 \leq i \leq n$. Este ultimo fato mostra que $\mathcal{Y}(\triangle)$ é fechada por conúcleos de monomorfismos. Logo pela Definição 3.4.4, $\mathcal{Y}(\triangle)$ é uma categoria corresolvente.

A afirmação 2 é uma conseqüência da Proposição 3.2.8, (4).

Dualmente temos o seguinte resultado.

Proposição 3.4.19. 1. Seja A uma álgebra coestandarmente estratificada. Então $\mathcal{W}(\nabla)$ é uma subcategoria resolvente de $\bmod A$.

2. $\mathcal{F}(\triangle) \subseteq \mathcal{W}(\nabla)$.

As inclusões das proposições anteriores podem ser próprias. Para ilustrar isto temos o seguinte exemplo, que foi apresentado em [26]. 
Exemplo 3.4.20. Para começar notemos que se uma álgebra $A$ é local de dimensão finita, então $A$ é estandarmente estratificada, coestandarmente estratificada, $\mathcal{F}(\triangle)=$ add $A$ e $\mathcal{F}(\nabla)=$ add $D\left(A_{A}\right)$. Se, além de local, $A$ é também autoinjetiva, como é o caso de $A=K[x] /\left\langle x^{2}\right\rangle$, então $\mathcal{F}(\triangle)=\mathcal{F}(\nabla)=$ add $A$. Nesta situação temos que $\mathcal{Y}(\triangle)=\mathcal{W}(\nabla)=\bmod A$ e portanto as inclusões $\mathcal{F}(\triangle) \subset \mathcal{W}(\nabla)$ e $\mathcal{F}(\nabla) \subset \mathcal{Y}(\triangle)$ são próprias.

\section{5 Álgebras estandarmente estratificadas e módulos inclinantes}

Nesta seção demonstraremos que se $A$ é uma álgebra estandarmente estratificada, então existe um $A$-módulo inclinante generalizado $T$ associado a $A$, tal que $\mathcal{F}(\triangle) \cap \mathcal{Y}(\triangle)=$ add $T$. Mostraremos também que o anel de endomorfismos $B=\operatorname{End}_{A}\left(T_{A}\right)$ é também uma $K$-álgebra estandarmente estratificada. Mais ainda, mostraremos que $\operatorname{End}_{B}\left(T^{\prime}\right)$ é Morita equivalente a $A$, onde $T^{\prime}$ é o $B$-módulo inclinante generalizado associado a $B$.

Os resultados apresentados nesta seção estão contidos no trabalho de C. $\mathrm{Xi}$, em [26], bem como [2] numa abordagem distinta.

Começamos então definindo o que é um módulo inclinante generalizado.

Definição 3.5.1. 1. Seja $A$ uma $K$-álgebra. Um A-módulo $T \in \bmod A$ é dito um módulo inclinante generalizado se satisfaz as seguintes condições:

(a) $p d T<\infty$;

(b) $\operatorname{Ext}_{A}^{i}(T, T)=0$, para todo inteiro $i \geq 1$; 
Álgebras estandarmente estratificadas e módulos inclinantes

(c) Existe uma seqüência exata do tipo

$$
0 \rightarrow A_{A} \rightarrow T_{0} \rightarrow T_{1} \rightarrow \cdots \rightarrow T_{s} \rightarrow 0,
$$

com $T_{i} \in$ add $T$, para $i=1,2, \ldots, s$.

2. Dualmente, um A-módulo $T$ é dito um A-módulo coinclinante generalizado se estão satisfeitas as condições:

(a) $i d T<\infty$;

(b) $\operatorname{Ext}_{A}^{i}(T, T)=0$, para todo inteiro $i \geq 1$;

(c) Existe uma seqüência exata do tipo

$$
0 \rightarrow T_{s} \rightarrow \cdots \rightarrow T_{1} \rightarrow T_{0} \rightarrow D\left({ }_{A} A\right) \rightarrow 0,
$$

com $T_{i} \in$ add $T$, para $i=1,2, \ldots, s$.

Antes de provarmos a existência de um $A$-módulo inclinante generalizado especial, ligado a categoria $\mathcal{F}(\triangle)$ quando $A$ é uma álgebra estandarmente estratificada, faremos algumas observações e daremos destaque a algumas propriedades da subcategoria $\mathcal{F}(\triangle) \cap \mathcal{Y}(\triangle)$.

Observação 3.5.2. Seja $A$ uma $K$-álgebra estandarmente estratificada. Então:

1. A categoria $\mathcal{Y}(\triangle) \cap \mathcal{F}(\triangle)$ é auto-ortogonal, isto é, $\operatorname{Ext}_{A}^{i}(W, Y)=0$, $\forall W, Y$ em $\mathcal{Y}(\triangle) \cap \mathcal{F}(\triangle)$ e $\forall i \geq 1$.

2. Para cada $X \in \mathcal{F}(\triangle)$, existe uma seqüência exata

$$
0 \longrightarrow X \longrightarrow W \longrightarrow X^{\prime} \longrightarrow 0,
$$

com $W \in \mathcal{Y}(\triangle) \cap \mathcal{F}(\triangle)$ e $X^{\prime} \in \mathcal{F}(\triangle)$. 
A primeira afirmação é um caso particular da Proposição 3.4.17. A segunda afirmaçã decorre da aplicação do Lema 2.1.10 $\operatorname{com} \theta=\triangle \mathrm{e}$ do fato de que $\mathcal{F}(\triangle)$ é fechado por extensões (Observação 2.1.2).

Existe um resultado análogo ao ítem (2) da observação acima para a categoria $\mathcal{Y}(\triangle)$, que enunciaremos no seguinte lema.

Lema 3.5.3. Seja A uma K-álgebra estandarmente estratificada. Então, para cada $Y \in \mathcal{Y}(\triangle)$, existe uma seqüência exata $0 \longrightarrow Y^{\prime} \longrightarrow W \longrightarrow Y \longrightarrow 0$, com $W \in \mathcal{Y}(\triangle) \cap \mathcal{F}(\triangle) e Y^{\prime} \in \mathcal{Y}(\triangle)$.

Demonstração. Sejam $Y \in \mathcal{Y}(\triangle)$ e $\gamma: P \rightarrow Y$ a sua cobertura projetiva em $\bmod A$. Como $A$ é uma álgebra estandarmente estratificada, em virtude do Corolário 3.3.5 e da Proposição 3.4.2, $P \in \mathcal{F}(\triangle)$. Consideremos a seqüência exata

$$
0 \longrightarrow \operatorname{ker}(\gamma) \longrightarrow P \longrightarrow Y \longrightarrow 0 .
$$

De outro lado, em virtude do Lema 2.1.10, existe uma seqüência exata da forma $0 \longrightarrow \operatorname{ker}(\gamma) \longrightarrow Y^{\prime} \longrightarrow X \longrightarrow 0$, com $Y^{\prime} \in \mathcal{Y}(\triangle)$ e $X \in \mathcal{F}(\triangle)$. Se

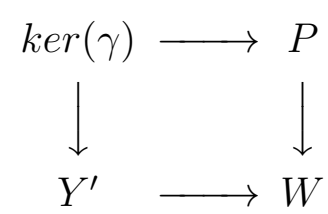

é o push-out de $Y^{\prime} \longleftarrow \operatorname{ker}(\gamma) \longrightarrow P$, então temos o seguinte diagrama co- 
mutativo

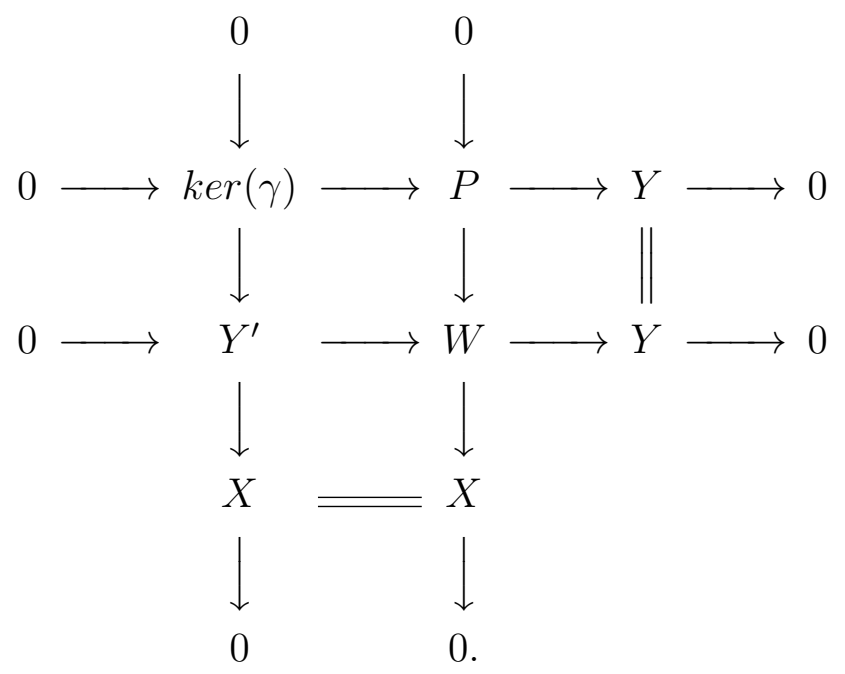

Desde que $P, X \in \mathcal{F}(\triangle), Y, Y^{\prime} \in \mathcal{Y}(\triangle)$ e tais categorias são fechadas por extensões, pela Observação 2.1 .2 e a Proposição 3.4.18, temos que $W$ esta em $\mathcal{F}(\triangle) \cap \mathcal{Y}(\triangle)$. Demonstramos pois que a seqüência exata

$$
0 \longrightarrow Y^{\prime} \longrightarrow W \longrightarrow Y \longrightarrow 0
$$

satisfaz as condições requeridas.

Antes de proseguir para a prova de que para uma álgebra estandarmente estratificada $A$, com relaçâo a seqüência $\triangle$, existe um $A$-módulo inclinante generalizado $T$ que determina os módulos Ext-injetivos em $\mathcal{F}(\triangle)$, lembraremos alguns fatos gerais sobre módulos.

Se $A$ é uma $K$-álgebra, pode-se provar (como "forma dual" à efetuada na Propasição 2.1, Cap. I, em [6]) que o funtor contravariante $F=$ $\operatorname{Hom}_{A}\left(\_, T_{A}\right): \bmod A \longrightarrow B-\bmod$ quando restrito a subcategoria $\operatorname{add}(T)$ estabelece uma equivalência contravariante entre a subcategoria $a d d(T)$ de $\bmod A$ e a subcategoria $\mathcal{P}(B)$ dos $B$-módulos projetivos à esquerda de $B$. 
Desta forma concluímos que o posto de $K_{0}(B)=K_{0}\left(\operatorname{End}_{A}(T)\right)$ é igual ao número de somandos indecomponíveis, não isomorfos, de $T$.

Observação 3.5.4. Vamos observar agora um importante fato sobre a teoría inclinante, que pode ser encontrado em [23], Corolário 1 do Teorema 1.19, que é o seguinte: Seja $A$ uma álgebra artiniana e $T_{A}$ um $A$-módulo inclinante generalizado. Então o número de classes de isomorfia dos $A$-módulos simples é igual ao número de classes dos $B$-módulos simples, onde $B=\operatorname{End}_{A}\left(T_{A}\right)$.

Estamos agora em condições de enunciar um dos mais importantes resultados desta seção, que é o teorema abaixo.

Teorema 3.5.5. Seja A uma K-álgebra estandarmente estratificada. Então existe um A-módulo inclinante generalizado $T$, único a menos da multiplicidade dos somandos diretos indecomponiveis, tal que add $T=\mathcal{F}(\triangle) \cap \mathcal{Y}(\triangle)$.

Demonstração. Seja $X \in \mathcal{F}(\triangle)$ qualquer. Como $A$ é estandarmente estratificada, então pela Observação 3.5.2 (2), existe uma seqüência exata

$$
0 \longrightarrow X_{-1} \longrightarrow W_{0} \longrightarrow X_{0} \longrightarrow 0
$$

onde $X_{-1}=X, W_{0} \in \mathcal{F}(\triangle) \cap \mathcal{Y}(\triangle)$ e $X_{0} \in \mathcal{F}(\triangle)$. Pela mesma razão, para $X_{0} \in \mathcal{F}(\triangle)$ existe uma seqüência exata da forma

$$
0 \longrightarrow X_{0} \longrightarrow W_{1} \longrightarrow X_{1} \longrightarrow 0
$$

com $W_{1} \in \mathcal{F}(\triangle) \cap \mathcal{Y}(\triangle)$ e $X_{1} \in \mathcal{F}(\triangle)$. Desta maneira construímos $n$ seqüências exatas da forma

$$
\epsilon_{i}=\left(0 \longrightarrow X_{i-1} \longrightarrow W_{i} \longrightarrow X_{i} \longrightarrow 0\right),
$$


com $W_{i} \in \mathcal{F}(\triangle) \cap \mathcal{Y}(\triangle)$ e $X_{i-1}$ e $X_{i}$ em $\mathcal{F}(\triangle), 0 \leq i \leq n$. Aplicando o funtor $\operatorname{Hom}_{A}\left(X_{n-1}, \ldots\right)$ a cada seqüência $\epsilon_{i}, i=1,2, \ldots, n$, obtemos a seqüência exata longa

$$
\begin{aligned}
& \operatorname{Ext}_{A}^{1}\left(X_{n-1}, W_{i}\right) \quad \longrightarrow \operatorname{Ext}_{A}^{1}\left(X_{n-1}, X_{i}\right) \quad \longrightarrow \operatorname{Ext}_{A}^{2}\left(X_{n-1}, X_{i-1}\right) \\
& \longrightarrow \operatorname{Ext}_{A}^{2}\left(X_{n-1}, W_{i}\right) \longrightarrow \operatorname{Ext}_{A}^{2}\left(X_{n-1}, X_{i}\right) \quad \longrightarrow \operatorname{Ext}_{A}^{3}\left(X_{n-1}, X_{i-1}\right) \\
& \longrightarrow \operatorname{Ext}_{A}^{j}\left(X_{n-1}, W_{i}\right) \longrightarrow \operatorname{Ext}_{A}^{j}\left(X_{n-1}, X_{i}\right) \longrightarrow \operatorname{Ext}_{A}^{j+1}\left(X_{n-1}, X_{i-1}\right) \\
& \vdots \quad \vdots \quad \vdots \\
& \longrightarrow \operatorname{Ext}_{A}^{n-1}\left(X_{n-1}, W_{i}\right) \longrightarrow \operatorname{Ext}_{A}^{n-1}\left(X_{n-1}, X_{i}\right) \longrightarrow \operatorname{Ext}_{A}^{n}\left(X_{n-1}, X_{i-1}\right) .
\end{aligned}
$$

Como $W_{i} \in \mathcal{F}(\triangle) \cap \mathcal{Y}(\triangle)$ e $X_{i}, X_{i-1} \in \mathcal{F}(\triangle)$, pela Observação 3.5 .2 (1), vale que $\operatorname{Ext}_{A}^{j}\left(X_{n-1}, W_{i}\right)=\operatorname{Ext}_{A}^{j+1}\left(X_{n-1}, W_{i}\right)=0$, para todo $j \geq 1$. Portanto $\operatorname{Ext}_{A}^{j}\left(X_{n-1}, X_{i}\right) \cong \operatorname{Ext}_{A}^{j+1}\left(X_{n-1}, X_{i-1}\right)=0$, para todo $j \geq 1$ e $i=1,2, \ldots, n$. Logo temos que

$$
\operatorname{Ext}_{A}^{1}\left(X_{n-1}, X_{n-2}\right) \cong \operatorname{Ext}_{A}^{2}\left(X_{n-1}, X_{n-3}\right) \cong \ldots \cong \operatorname{Ext}_{A}^{n}\left(X_{n-1}, X_{-1}\right)
$$

Desde que $X_{n-1} \in \mathcal{F}(\triangle)$, então, em virtude do Corolário 3.4.14, pd $X_{n-1} \leq$ $n-1$ e $\operatorname{logo} \operatorname{Ext}_{A}^{n}\left(X_{n-1}, X_{-1}\right) \cong \operatorname{Ext}_{A}^{1}\left(X_{n-1}, X_{n-2}\right)=0$. Assim a seqüência exata

$$
\epsilon_{n-1}=\left(0 \longrightarrow X_{n-2} \longrightarrow W_{n-1} \longrightarrow X_{n-1} \longrightarrow 0\right)
$$

cinde, o que implica que $X_{n-2}$ é um somando direto de $W_{n-1} \in \mathcal{F}(\triangle) \cap \mathcal{Y}(\triangle)$ e, portanto, que $X_{n-2} \in \mathcal{F}(\triangle) \cap \mathcal{Y}(\triangle)$.

Usando as seqüências $\epsilon_{i}$ construímos uma nova seqüência exata da seguinte forma

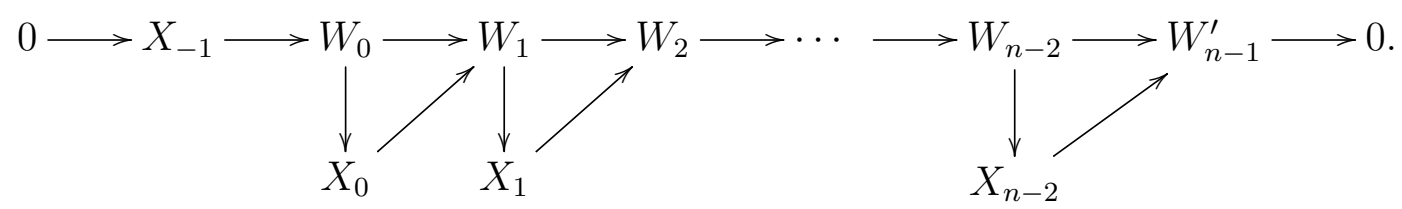


Notemos que na seqüência obtida

$$
0 \longrightarrow X_{-1} \longrightarrow W_{0} \longrightarrow \cdots \longrightarrow W_{n-2} \longrightarrow W_{n-1}^{\prime} \longrightarrow 0
$$

tanto $W_{n-1}^{\prime}=X_{n-2}$ quanto $W_{i}$, para $0 \leq i \leq n-2$, estão em $\mathcal{F}(\triangle) \cap \mathcal{Y}(\triangle)$.

Até aqui temos construído para cada $X=X_{-1} \in \mathcal{F}(\triangle)$ uma seqüência do tipo (3.4). Em particular, como $A_{A} \in \mathcal{F}(\triangle)$ existe uma seqüência da forma

$$
0 \longrightarrow A_{A} \longrightarrow W_{0} \longrightarrow \cdots \longrightarrow W_{n-2} \longrightarrow W_{n-1} \longrightarrow 0
$$

com $W_{i} \in \mathcal{F}(\triangle) \cap \mathcal{Y}(\triangle)$, para $0 \leq i \leq n-1$.

Seja o $A$-módulo $T^{\prime}=\oplus_{i=0}^{n-1} W_{i}$. Como $T^{\prime} \in \mathcal{F}(\triangle) \cap \mathcal{Y}(\triangle)$, pelo Corolário 3.4.14 resulta que $p d T^{\prime} \leq n-1$. De outro lado, pela Observação 3.5.2 (1), temos que $\operatorname{Ext}_{A}^{j}\left(T^{\prime}, T^{\prime}\right)=0$, para todo $j \geq 1$. Assim, junto com a seqüência em (3.5) temos que $T^{\prime}$ é um $A$-módulo inclinante generalizado.

Mostremos agora que $\mathcal{F}(\triangle) \cap \mathcal{Y}(\triangle)=\operatorname{add}\left(T^{\prime}\right)$. Pela construção de $T^{\prime}=$ $\oplus_{i=0}^{n-1} W_{i}$, com $W_{i} \in \mathcal{F}(\triangle) \cap \mathcal{Y}(\triangle)$, temos claramente que add $\left(T^{\prime}\right) \subseteq \mathcal{F}(\triangle) \cap$ $\mathcal{Y}(\triangle)$. Para a inclusão contraria, seja $M \in \mathcal{F}(\triangle) \cap \mathcal{Y}(\triangle)$. É fácil ver que $T_{A}^{\prime} \oplus M_{A}$ é também um $A$-módulo inclinante generalizado. Assim, temos pela Observação 3.5.4 que o número de $B=\operatorname{End}_{A}\left(T^{\prime}\right)$-módulos simples, é igual ao número de $\bar{B}=\operatorname{End}_{A}\left(T^{\prime} \oplus M\right)$-módulos simples, não isomorfos. De outro lado este número, pela observação feita anteriormente é igual ao número de somandos diretos de $T^{\prime}$. Portanto $M \in \operatorname{add}\left(T^{\prime}\right)$ e esta aprovado que $\mathcal{F}(\triangle) \cap \mathcal{Y}(\triangle)=\operatorname{add}\left(T^{\prime}\right)$. Finalmente observemos que se existir um $A$ módulo inclinante generalizado $T^{\prime \prime}$ tal que $\mathcal{F}(\triangle) \cap \mathcal{Y}(\triangle)=\operatorname{add}\left(T^{\prime \prime}\right)$, teremos que eles têm os mesmos somandos diretos indecomponíveis e difierem na multiplicidade destes somandos. 
Álgebras estandarmente estratificadas e módulos inclinantes

A unicidade, a menos da multiplicidade, garantida pelo terema anterior motiva a seguinte definição.

Definição 3.5.6. Seja A uma K-álgebra estandarmente estratificada. O A-módulo inclinante generalizado $T$, com seus somandos diretos indecomponíveis não isomorfos, de multiplicidade um, tal que $\mathcal{F}(\triangle) \cap \mathcal{Y}(\triangle)=\operatorname{add}(T)$ é denominado módulo inclinante característico associado a $\triangle$.

O passo a seguir é demonstrar que o módulo inclinante característico de uma álgebra quase-hereditária é também coinclinante. Para isto enunciaremos uma série de resultados e definições que usaremos em tal demonstração.

Seja $A$ uma $K$-álgebra. Denotamos por $\mathcal{P}^{<\infty}(A)$ e $\mathcal{I}^{<\infty}(\mathcal{A})$, respectivamente, as subcategorias de $\bmod A$ formadas pelos $A$-módulos $X$ tais que $p d X<\infty$ e pelos $A$-módulos $Y$ tais que $i d Y<\infty$. Com esta notação, definimos a dimensão projetiva finitística de $A$, que denotaremos por pfd $A$, como o supremo das dimensões projetivas dos objetos de $\mathcal{P}^{<\infty} A$, ou seja, $p f d A=\sup \left\{p d X: X \in \mathcal{P}^{<\infty}(A)\right\}$.

Seguintes dois lemas foram provados em [5].

Proposição 3.5.7. Seja $A$ uma álgebra tal que id $A_{A}<\infty$ e que id ${ }_{A} A<\infty$. Então id $A_{A}=i d_{A} A$ e $\mathcal{I}^{<\infty}(A)=\mathcal{P}^{<\infty}(A)$.

Proposição 3.5.8. Se id $A_{A}<\infty$, então id $A A<\infty$ se, e somente se, a dimensão projetiva finitística de A é finita.

O teorema abaixo, cuja prova pode ser encontrada em [1], garante que a 
dimensão projetiva finitística de uma álgebra estandarmente estratificada é finita.

Teorema 3.5.9. Seja A uma K-álgebra estandarmente estratificada. Então pfd $A \leq 2 n-2$, onde $n$ é o posto de $K_{0}(A)$.

Por fim enunciamos uma proposição que fornece uma condição suficinte e necessaria para que o módulo inclinante característico seja também coinclinante.

Proposição 3.5.10. Sejam A uma K-álgebra estandarmente estratificada e T seu módulo inclinante característico associado. As seguintes condições são equivalentes:

1. T é um A-módulo coinclinante generalizado.

2. id $A_{A}<\infty$.

Demonstração. Demonstremos que (1) implica (2). Suponhamos que $T$ é um $A$-módulo coinclinante generalizado. Logo existe uma seqüência exata

$$
0 \longrightarrow T_{s} \longrightarrow T_{s-1} \longrightarrow \cdots \longrightarrow T_{0} \longrightarrow D\left({ }_{A} A\right) \longrightarrow 0
$$

com $T_{i} \in \mathcal{F}(\triangle) \cap \mathcal{Y}(\triangle), 0 \leq i \leq s$. Como cada $T_{i} \in \mathcal{F}(\triangle)$, então, pelo Corolário 3.4.14, $p d T_{i}<\infty$ e, portanto, $p d D\left({ }_{A} A\right)<\infty$, ou equivalentemente id $A_{A}<\infty$. Assim (1) implica (2) está provada.

Para mostrar que (2) implica (1), suponhamos que id $A_{A}<\infty$. Então $p d D\left({ }_{A} A\right)=m<\infty$. Desde que $D\left({ }_{A} A\right) \in \mathcal{Y}(\triangle)$, aplicando iteradamente o Lema 3.5.3, como foi feito na prova do Teorema 3.5.5, obtemos uma família 
$\left(\epsilon_{i}\right), i=1,2, \ldots, m$, de sequiências exatas da forma:

$$
\epsilon_{i}=\left(0 \longrightarrow Y_{i} \longrightarrow W_{i} \longrightarrow Y_{i-1} \longrightarrow 0\right)
$$

com $Y_{i-1}$ e $Y_{i}$ em $\mathcal{Y}(\triangle)$, sendo $Y_{-1}=D\left({ }_{A} A\right)$, e $W_{i} \in \mathcal{F}(\triangle) \cap \mathcal{Y}(\triangle)$. Também como na prova do Teorema 3.5.5, utilizando a Observação 3.5.2, obtemos que a seqüência $\epsilon_{m}$ cinde e que $Y_{m-1} \in \mathcal{F}(\triangle) \cap \mathcal{Y}(\triangle)$. Dessa forma, construímos a seqüência exata

$$
0 \longrightarrow W_{m}^{\prime} \longrightarrow W_{m-1} \longrightarrow \cdots \longrightarrow W_{0} \longrightarrow D\left({ }_{A} A\right) \longrightarrow 0,
$$

onde $W_{m}^{\prime}=Y_{m-1}$. Como $W_{m}^{\prime}$ e cada $W_{i}$ estão em $\mathcal{F}(\triangle) \cap \mathcal{Y}(\triangle)=\operatorname{add} T$ (Teorema 3.5.5), resulta que a seqüência em (3.6) e o fato de que $\operatorname{Ext}_{A}^{i}(T, T)=0$, para todo $i \geq 1$ (Observação 3.5.2), garantem que as condições (b) e (c) da Definição 3.5.1 (2) se verificam. Portanto, para mostrar que $T$ é um $A$-módulo coinclinante basta verificar que $i d T<\infty$. De um lado, como $T \in \mathcal{F}(\triangle) \cap \mathcal{Y}(\triangle)$ resulta do Corolário 3.4.14 que $p d T<\infty$, ou seja $T \in \mathcal{P}^{<\infty}(A)$. De outro lado, o Teorema 3.5.9 estabelece que a dimensão finitística de $A$ é finita, o que junto com a hipótese de que id $A<\infty$ resulta, pela Proposição 3.5.8, em que $\mathcal{P}^{<\infty}(A)=\mathcal{I}^{<\infty}(A)$, ou seja que $T$ tem dimensão injetiva finita.

Corolário 3.5.11. Seja A uma K-álgebra quase-hereditária. Então o Amódulo inclinante característico associado $T$ é também um A-módulo coinclinante generalizado.

Demonstração. Da Proposição 3.4.13 (3) vem que $i d A_{A}<\infty$ e portanto a Proposição 3.5.10 garante a afirmação. 
Álgebras estandarmente estratificadas e módulos inclinantes

No Corolário 3.5.11 a condição de que a álgebra seja quase-hereditária é essencial. Para verificar isto temos o seguinte exemplo dado em [26].

Exemplo 3.5.12. Sejam $K$ um corpo algebricamente fechado e a $K$-álgebra $A=K[x, y] /\langle x, y\rangle^{2}$. Como $A$ é local, então ela é estandarmente estratificada. Notese que $A \cong K Q / I$, onde $Q$ é o carcás

$$
{ }^{\alpha} C_{1} \cdot{ }^{\beta}
$$

e $I=\left\langle\alpha^{2}, \beta^{2}, \alpha \beta, \beta \alpha\right\rangle$. As correspondentes representações associadas ao módulo projetivo $P_{1}$ e ao módulo injetivo $I_{1}$ são, respectivamente

$$
P_{1}:\left[\begin{array}{lll}
0 & 0 & 0 \\
0 & 0 & 0 \\
1 & 0 & 0
\end{array}\right]\left(\cdot K^{3}\right)\left[\begin{array}{lll}
0 & 0 & 0 \\
1 & 0 & 0 \\
0 & 0 & 0
\end{array}\right] \quad I_{1}:\left[\begin{array}{ccc}
1 & 0 & 0 \\
0 & 0 & 0 \\
0 & 0 & 0
\end{array}\right] C\left(K^{3}\right)\left[\begin{array}{lll}
1 & 0 & 0 \\
0 & 0 & 0 \\
0 & 0 & 0
\end{array}\right]
$$

Claramente $I_{1} \nsucceq P_{1}$. Assim temos que $\mathcal{F}(\triangle)=\operatorname{add} A$ e $\mathcal{Y}(\triangle)=\bmod A$. Portanto $\mathcal{F}(\triangle) \cap \mathcal{Y}(\triangle)=\mathcal{F}(\triangle)=$ add $A$. Mas como $A$ é local os módulos de dimensão injetiva finita são os $A$-módulos injetivos. Desde que $I_{1} \notin$ $\mathcal{F}(\triangle) \cap \mathcal{Y}(\triangle)$, então não existe nenhum módulo de dimensão injetiva finita em $\mathcal{F}(\triangle) \cap \mathcal{Y}(\triangle)$ e, portanto, não existe módulo coinclinante $T$ tal que $\mathcal{F}(\triangle) \cap \mathcal{Y}(\triangle)=\operatorname{add} T$.

A seguinte proposição garante a existência de uma família de sequências exatas relacionadas com os módulos indecomponíveis de $\mathcal{F}(\triangle) \cap \mathcal{Y}(\triangle)$.

Proposição 3.5.13. Seja A uma K-álgebra estandarmente estratificada. Então, para cada $1 \leq i \leq n$, existe uma seqüência exata curta

$$
0 \longrightarrow \triangle_{i} \stackrel{\beta(i)}{\longrightarrow} T(i) \longrightarrow X(i) \longrightarrow 0,
$$

onde $\beta(i)$ é uma $\mathcal{Y}(\triangle)$-aproximação minimal à esquerda de $\triangle_{i}, X(i) \in$ $\mathcal{F}\left(\triangle_{1}, \ldots, \triangle_{i-1}\right), T(i) \in \mathcal{F}(\triangle) \cap \mathcal{Y}(\triangle)$ é indecomponivel e $\mathcal{F}(\triangle) \cap \mathcal{Y}(\triangle)=$ $\operatorname{add}\left(\oplus_{i=1}^{n} T(i)\right)$. 
Demonstração. Seja um $i=1, \ldots, n$ fixado. $\operatorname{Como} \operatorname{Ext}_{A}^{1}\left(\triangle_{j}, \triangle_{i}\right)=0$, para todo $j \geq i$, então pelo Lema 2.1.9 existe uma seqüência exata curta

$$
0 \longrightarrow \triangle_{i} \stackrel{\beta^{\prime}}{\longrightarrow} T^{\prime}(i) \stackrel{\pi^{\prime}}{\longrightarrow} X^{\prime}(i) \longrightarrow 0
$$

com $T^{\prime}(i) \in \mathcal{Y}(\triangle)$ e $X^{\prime}(i) \in \mathcal{F}\left(\triangle_{1}, \ldots, \triangle_{i-1}\right)$. Desde que $\triangle_{i}$ e $X^{\prime}(i)$ estão em $\mathcal{F}(\triangle)$, que é uma subcategoria fechada por extensões (Observação 2.1.2), temos também que $T^{\prime}(i) \in \mathcal{Y}(\triangle) \cap \mathcal{F}(\triangle)$.

Por outro lado, pelo Teorema 2.4, Cap. 1 em [6], existe uma decomposição de $T^{\prime}(i)=T(i) \oplus T^{\prime \prime}(i)$ e, em conseqüência por ser $\pi^{\prime}$ um epimorfismo, uma decomposição de $X^{\prime}(i)=X(i) \oplus X^{\prime \prime}(i)$ tal que a sequência acima é reescrita na forma:

$$
0 \longrightarrow \Delta_{i} \stackrel{\left(\begin{array}{c}
\beta(i) \\
0
\end{array}\right)}{\longrightarrow} T(i) \oplus T^{\prime \prime}(i) \stackrel{\left(\begin{array}{cc}
\pi(i) & 0 \\
0 & \pi^{\prime \prime}(i)
\end{array}\right)}{\longrightarrow} X(i) \oplus X^{\prime \prime}(i) \longrightarrow 0
$$

onde $\beta(i)$ é a versão minimal à esquerda de $\beta^{\prime}(i)$. Consideremos então a sequência exata:

$$
0 \longrightarrow \triangle_{i} \stackrel{\beta(i)}{\longrightarrow} T(i) \stackrel{\pi(i)}{\longrightarrow} X(i) \longrightarrow 0 .
$$

Uma vez que as categorias $\mathcal{Y}(\triangle)$ e $\mathcal{F}(\triangle)$ são fechadas por somandos diretos (Corolário 3.3.5) temos que $T(i) \in \mathcal{Y}(\triangle) \cap \mathcal{F}(\triangle)$ e $X(i) \in \mathcal{F}\left(\triangle_{1}, \ldots, \triangle_{i-1}\right)$. A verificação de que $\beta(i)$ é uma $\mathcal{Y}(\triangle)$-aproximação à esquerda de $\triangle_{i}$ é de forma dual à feita na prova do Lema 2.1.6 e, por isso, a omitiremos aqui.

Demonstremos então que $T(i)$ é indecomponível. Para tanto, vamos supor que $T(i)=T_{1} \oplus T_{2}$, com $T_{j} \neq 0$, para $j=1,2$. Se denotamos $\beta(i)=\left(\beta_{1}, \beta_{2}\right)^{t}$ e $\pi(i)=\left(\pi_{1}, \pi_{2}\right)$, onde $\beta_{j}: \triangle_{i} \longrightarrow T_{j}$ e $\pi_{j}: T_{j} \longrightarrow X(i)$, para $j=1,2$, temos que $\beta_{j} \neq 0$, para toda $j=1,2$, pois $\beta(i)$ é minimal. De outro lado, observemos que $\triangle_{i} \cong \operatorname{Im} \beta(i)=\left\{\left(t_{1}, t_{2}\right) \in T_{1} \oplus T_{2}:-\pi_{1}\left(t_{1}\right)=\pi_{2}\left(t_{2}\right)\right\}$. 
Portanto, temos o seguinte diagrama de pull-back:

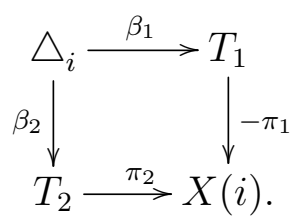

Como $X(i) \in \mathcal{F}\left(\triangle_{1}, \ldots, \triangle_{i-1}\right)$, temos então que $\operatorname{Hom}_{A}\left(\triangle_{i}, X(i)\right)=0$. Portanto $\pi_{1} \beta_{1}=\pi_{2} \beta_{2}=0$. Assim do fato de que $-\pi_{1} \beta_{1}=\pi_{2} 0=0$, resulta da propriedade universal do pull-back que existe um homomorfismo $\alpha: \triangle_{i} \longrightarrow \triangle_{i}$ tal que $\beta_{1}=\beta_{1} \alpha$ e $\beta_{2} \alpha=0$. Claramente $\alpha$ não é invertível e como $\operatorname{End}_{A}\left(\triangle_{i}\right)$ é um local, (pois $\triangle_{i}$ é um $A$-módulo indecomponivel) portanto $\alpha$ é nilpotente. Indutivamente é fácil ver que $\beta_{1} \alpha^{m}=\beta_{1}$, para qualquer inteiro positivo $m \geq 1$. Em particular, se $m$ é igual ao índice de nilpotência de $\alpha$ então $\beta_{1}=0$, o qual contradiz o fato de que $\beta_{1} \neq 0$. Portanto, $T(i)$ é indecomponível.

A sequiência (3.7), garante que os fatores de composição de $T(i)$ são os fatores de composição de $\triangle_{i}$ e os de $X(i) \in \mathcal{F}\left(\triangle_{1}, \ldots, \triangle_{i-1}\right)$, que são os simples $S_{j}$ com $j \leq i$, sendo que $S_{i}$ deve ocorrer pelo menos uma vez. Dessa forma temos que para $i \neq j, T(i)$ e $T(j)$ são não isomorfos pois $\beta$ é não nula. Assim os módulos $T(i), 1 \leq i \leq n$, são dois a dois não isomorfos. Em conseqüência, obtemos pois que $\mathcal{F}(\triangle) \cap \mathcal{Y}(\triangle)=\operatorname{add}\left(\oplus_{i=1}^{n} T(i)\right)$.

Nosso intuito agora é estudar o anel dos endomorfismos $\operatorname{End}_{A}(T)$ do $A$ módulo inclinante associado a uma álgebra estandarmente estratificada $A$. Começamos com algumas observações gerais. 
Álgebras estandarmente estratificadas e módulos inclinantes

Observação 3.5.14. Relembraremos aqui alguns fatos bem conhecidos, cuja verificação é bastante simples e por isso será omitida, e que serão bem uteis para o lema a seguir.

1. Se $A$ é um anel qualquer e $T$ é um $A$-módulo à direita, então $T$ admite uma estrutura natural de $B$-módulo à esquerda, onde $B=\operatorname{End}_{A}(T)$, que é dada por $f . x:=f(x)$, para $f \in B$ e $x \in T$. Mas do que isso, $T$ é um $B$-A-bimódulo.

2. Se $X$ é um $A$-módulo à direita, então $\operatorname{Hom}_{A}\left(X_{A, B} T_{A}\right)$ tem uma estrutura natural de $B$-módulo à esquerda, dada por $\beta * f=\beta \circ f$, para todo $\beta \in B$ e para todo $f \in \operatorname{Hom}_{A}\left(X_{A, B} T_{A}\right)$. Dessa forma podemos considerar o grupo abeliano $G=\operatorname{Hom}_{B}\left(\operatorname{Hom}_{A}\left(X_{A, B} T_{A}\right){ }_{B} T_{A}\right)$ sobre o qual também é possível definir uma estrutura de $A$-módulo à direita, que é dada por $(\psi \odot \alpha)(g)=\psi(g) \alpha$, para $\psi \in G, \alpha \in A$ e $g \in \operatorname{Hom}_{A}\left(X_{A}, T_{A}\right)$.

3. Sejam $R$ e $S$ dois anéis quaisquer. Consideremos $M$ um $R$-módulo à esquerda, $N$ um $S$-módulo à direita e $U$ um $R-S$ bimódulo. Então

$$
\operatorname{Hom}_{R}\left({ }_{R} M, \operatorname{Hom}_{S}\left(N_{S}, R U_{S}\right)\right) \cong \operatorname{Hom}_{S}\left(N_{S}, \operatorname{Hom}_{R}\left({ }_{R} M,{ }_{R} U_{S}\right)\right),
$$

como grupos abelianos.

Observação 3.5.15. Sejam $A$ uma $K$-álgebra estandarmente estratificada e $T$ o $A$-módulo inclinante associado. Então o funtor $F=\operatorname{Hom}_{A}(\ldots, T)$ : $\bmod A \longrightarrow B \bmod$ é exato em $\mathcal{F}(\triangle)$ (este fato é decorrência imediata da Proposição de $\mathcal{Y}(\triangle))$.

Para estabelecer algumas relações entre os $A$-módulos de $\mathcal{F}(\triangle)$ e as suas imagens a través do funtor $F$ em $B$ mod, fixaremos antes algumas notações. 
Álgebras estandarmente estratificadas e módulos inclinantes

Consideremos $\triangle=\left(\triangle_{1}, \triangle_{2}, \ldots, \triangle_{n}\right)$ a seqüência de módulos estandares (à direita) com relação a qual $A$ é estandarmente estratificada. Vamos denotar por $\triangle_{i}^{\prime}$ o $B$-módulo à esquerda $\operatorname{Hom}_{A}\left(\triangle_{n-i+1}, T\right)$ e por $\triangle^{\prime}$ a seqüência $\left(\triangle_{1}^{\prime}, \triangle_{2}^{\prime}, \ldots, \triangle_{n}^{\prime}\right)$ de $B$-módulos à esquerda.

Com estas notações, temos o seguinte lema que relaciona os $A$-módulos em $\mathcal{F}(\triangle)$ e as suas imagens em $B \bmod$ pelo funtor $F$.

Lema 3.5.16. Sejam A uma $K$-álgebra estandarmente estratificada e $T$ o Amódulo inclinante característico associado. Consideremos a $K$-álgebra $B=$ $\operatorname{End}_{A}(T)$. Então:

1. Para cada $X \in \mathcal{F}(\triangle)$, a função avaliação

$$
e_{X}: X_{A} \longrightarrow \operatorname{Hom}_{B}\left(\operatorname{Hom}_{A}\left(X_{A},_{B} T_{A}\right){ }_{B} T\right)
$$

é um isomorfismo de A-módulos (à direita).

2. $O$ funtor $F=\operatorname{Hom}_{A}(\ldots, T)$ é uma equivalência de categorias entre $\mathcal{F}(\triangle)$ e sua imagem $F(\mathcal{F}(\triangle))$, que é uma subcategoria de $B$ - mod.

3. Para cada $X \in \mathcal{F}(\triangle)$, sua imagem $F(X) \in B$ mod admite uma $\triangle^{\prime}$ filtração.

Demonstração. Para a prova de (1) observemos que o isomorfismo vale para $X=T$. Então para os somandos diretos de $T$, portanto para todos os módulos em add $T$.

Suponhamos que $X \in \mathcal{F}(\triangle)$. Pela prova do Teorema 3.5.5, via a observação 3.5.2 (2), obtemos uma família de seqüências exatas $\left\{\epsilon_{i}\right\}_{i=1}^{n-1}$, com $\epsilon_{1}:\left(0 \longrightarrow X \longrightarrow T_{0} \longrightarrow X_{0} \longrightarrow 0\right)$ e $\epsilon_{i}:\left(0 \longrightarrow X_{i-1} \longrightarrow T_{i} \longrightarrow X_{i} \longrightarrow 0\right)$, onde $T_{i} \in \mathcal{F}(\triangle) \cap \mathcal{Y}(\triangle)=\operatorname{add}(T), X_{n-1}=T_{n-1}$ e $X_{i} \in \mathcal{F}(\triangle)$, para $i=0,1, \ldots, n-1$. 
Consideramos a seqüência $\epsilon_{n-2}$. Se denotamos $\operatorname{Hom}_{A}\left(X_{A}, T_{A}\right)$ por $X^{*} \mathrm{e}$ aplicamos o funtor $\operatorname{Hom}_{A}(\ldots, T)$ a $\epsilon_{n-2}$, obtemos a seqüência exata curta $0 \longrightarrow T_{n-1}^{*} \longrightarrow T_{n-2}^{*} \longrightarrow X_{n-3}^{*} \longrightarrow 0$, pois o funtor $\operatorname{Hom}_{A}(\longrightarrow, T)$ é exato em $\mathcal{F}(\triangle)$ pela Observação 3.5.15.

Se aplicamos o funtor $\operatorname{Hom}_{B}(\ldots, B T)$ (que será denotado também por $*$ ) à seqüência acima, obtemos o seguinte diagrama comutativo

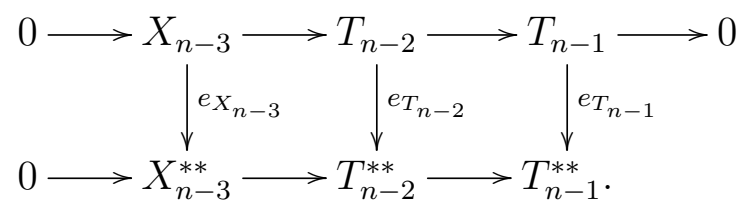

Desde que $e_{T_{n-2}}$ e $e_{T_{n-1}}$ são isomorfismos, então $e_{X_{n-3}}$ é também um isomorfismo. Usando o mesmo argumento repetidamente a cada uma das seqüências $\epsilon_{n-3}, \ldots, \epsilon_{1}$, nesta ordem, obtemos através da seqüência $\epsilon_{1}$ que $e_{X}$ é um isomorfismo, o que prova (1).

Para (2), basta provar que o funtor $\operatorname{Hom}_{A}\left(-, T_{A}\right): \bmod A \longrightarrow B-\bmod$ é um funtor fiel e pleno de $\mathcal{F}(\triangle)$ na subcategoria de $B$-mod formada pela sua imagem. Assim o que resta provar é que $\operatorname{Hom}_{A}(X, Y) \cong \operatorname{Hom}_{B}\left(Y^{*}, X^{*}\right)$, para quaisquer $X, Y \in \mathcal{F}(\triangle)$ (como grupos abelianos).

Desde que ${ }_{B} T_{A}$ é um $B-A$-bimódulo e que $Y^{*}$ e $X^{*}$ são $B$-módulos à esquerda, então podemos aplicar a Observação 3.5.14 escolhendo $R=B$, $S=A, U={ }_{B} T_{A}, M=Y^{*}$ e $N=X$, obtendo assim que

$$
\operatorname{Hom}_{B}\left(Y^{*}, X^{*}\right)=\operatorname{Hom}_{B}\left(Y^{*}, \operatorname{Hom}_{A}(X, T)\right) \cong \operatorname{Hom}_{A}\left(X, \operatorname{Hom}_{B}\left(Y^{*}, T\right)\right),
$$

mas, pela parte (1), temos que $Y_{A} \cong \operatorname{Hom}_{B}\left(\operatorname{Hom}_{A}(Y, T), T\right)_{A}$, e portanto concluímos que $\operatorname{Hom}_{B}\left(Y^{*}, X^{*}\right) \cong \operatorname{Hom}_{A}(X, Y)$ (como grupos abelianos). 
Álgebras estandarmente estratificadas e módulos inclinantes

Seja $X \in \mathcal{F}(\triangle)$. Para provar (3) usaremos indução sobre o número de elementos de $\operatorname{Supp}_{\triangle}(X)=\left\{i \in\{1,2, \ldots, n\}:\left[X: \triangle_{i}\right] \neq 0\right\}$. Se $\left|\operatorname{Supp}_{\triangle}(X)\right|=$ 1 , então $X \cong \triangle_{j}^{t}$, para algum $j \in\{1,2, \ldots, n\}$ e algum $t \geq 1$. Portanto $\operatorname{Hom}_{A}(X, T) \cong \operatorname{Hom}_{A}\left(\triangle_{j}^{t}, T\right) \cong\left(\triangle_{n-j+1}^{\prime}\right)^{t} . \quad \operatorname{Logo} F(X) \cong\left(\triangle_{n-j+1}^{\prime}\right)^{t}$ tem uma $\triangle^{\prime}$-filtração.

Suponhamos que a afirmação é verdadeira para todo $A$-módulo $M \in$ $\mathcal{F}(\triangle)$ tal que $1 \leq\left|\operatorname{Supp}_{\triangle}(M)\right|<m$. Seja $X \in \mathcal{F}(\triangle)$ tal que $\operatorname{Supp}_{\triangle}(X)=$ $\left\{i_{1}, i_{2}, \ldots, i_{m}\right\}$, com $2 \leq m \leq n$, e $i_{m}=\max \operatorname{Supp}_{\Delta}(X)$. Pela Observação 3.3.3, existe um submódulo $M \subset X$ tal que $\operatorname{Supp}_{\triangle}(X / M)=\left\{i_{1}, i_{2}, \ldots, i_{m-1}\right\}$, e a sequiência exata $0 \longrightarrow M \longrightarrow X \longrightarrow X / M \longrightarrow 0$ está em $\mathcal{F}(\triangle)$. Aplicando o funtor $F$ nesta seqüência, obtemos a seguinte seqüência em $B$ mod:

$$
0 \longrightarrow F(X / M) \longrightarrow F(X) \longrightarrow F(M) \cong\left(\triangle_{n-i_{m}+1}^{\prime}\right)^{t} \longrightarrow 0 .
$$

Como $\left|\operatorname{Supp}_{\triangle}(X / M)\right|<m$, então, pela hipótese de indução, resulta que $F(X / M)$ admite uma $\triangle^{\prime}$-filtração, o que junto com a ultima sequiência exata leva à conclusão de que $F(X)$ também admite uma $\triangle^{\prime}$-filtraçâo.

Com este lema e lembrando a observação anterior de que $\operatorname{Hom}_{A}(T(i), T)$, $i=1,2, \ldots, n$, onde $T=\oplus_{i=1}^{n} T(i)$ é o $A$-módulo inclinante característico, são os representantes dos $B$-módulos projetivos à esquerda, indecomponíveis, provaremos no teorema que segue que a álgebra $B=\operatorname{End}_{A}(T)$ é uma álgebra estandarmente estratificada com relação à ordem oposta na qual $A$ o é.

Proposição 3.5.17. Sejam A uma álgebra estandarmente estratificada, relativa à seqüência de A-módulos (à direita) estandares $\triangle=\left(\triangle_{1}, \triangle_{2}, \ldots, \triangle_{n}\right.$ ) 
Álgebras estandarmente estratificadas e módulos inclinantes

e $T=\oplus_{j=1}^{n} T(j)$ o A-módulo inclinante característico. Então a $K$-álgebra $B=\operatorname{End}_{A}(T)$ é uma álgebra estandarmente estratificada com seqüência de B-módulos (à esquerda) estandares $\triangle^{\prime}=\left(\triangle_{1}^{\prime}, \triangle_{2}^{\prime}, \ldots, \triangle_{n}^{\prime}\right)$, onde $\triangle_{i}^{\prime}=$ $\operatorname{Hom}_{A}\left(\triangle_{n-i+1}, T\right)$.

Demonstração. Seja $\triangle=\left(\triangle_{1}, \triangle_{2}, \ldots, \triangle_{n}\right)$ a seqüência de $A$-módulos (à direita) estandares, relativa a uma ordem fixada, para qual $A$ é uma álgebra estandarmente estratificada. Para cada $i=1,2, \ldots, n$, denotamos por $P^{\prime}(i)$ o $B$-módulo (à esquerda) $\operatorname{Hom}_{A}(T(n-i+1), T)$, que segundo a observação feita apos do Lema 3.5.16, é projetivo e indecomponível. Fixemos a seqüência do conjunto dos idempotentes, primitivos e ortogonais de $B$ segundo a seqüência ordenada dos $B$-módulos projetivos indecomponíveis $\left(P^{\prime}(1), P^{\prime}(2), \ldots, P^{\prime}(n)\right)$. Mostremos que a seqüência $\triangle^{\prime}=$ $\left(\triangle_{1}^{\prime}, \triangle_{2}^{\prime}, \ldots, \triangle_{n}^{\prime}\right)$, onde $\triangle_{i}^{\prime}=\operatorname{Hom}_{A}\left(\triangle_{n-i+1}, T\right)$ é a sequiência de $B$-módulos (à esquerda) estandares. Ou seja, vamos mostrar que para cada $i=1,2, \ldots, n$, $\triangle_{i}^{\prime}$ é o quociente de $P^{\prime}(i)$ pelo submódulo gerado pelas imagens dos $B$ homomorfismos de $P^{\prime}(j)$ para $P^{\prime}(i)$, com $j>i$.

Primeiramente, para um $i=1,2, \ldots, n$ fixado, pela Proposição 3.5.13, existe uma seqüência exata em $\mathcal{F}(\triangle)$

$$
0 \longrightarrow \triangle_{i^{\prime}} \stackrel{\beta\left(i^{\prime}\right)}{\longrightarrow} T\left(i^{\prime}\right) \longrightarrow X\left(i^{\prime}\right) \longrightarrow 0,
$$

onde $i^{\prime}=n-i+1, \beta\left(i^{\prime}\right)$ é uma $\mathcal{Y}(\triangle)$-aproximação minimal à esquerda de $\triangle_{i^{\prime}}$ e $X\left(i^{\prime}\right) \in \mathcal{F}\left(\triangle_{1}, \triangle_{2}, \ldots, \triangle_{i^{\prime}-1}\right)$; além disso, os fatores de composição de $T\left(i^{\prime}\right)$ são $A$-módulos simples $S_{m}$, com $m \leq i^{\prime}=n-i+1$. Aplicando o funtor $F=\operatorname{Hom}_{A}(\ldots, T)$ à sequiência exata acima e usando o fato de que $F$ é exato 
Álgebras estandarmente estratificadas e módulos inclinantes

em $\mathcal{F}(\triangle)$, obtemos a seguinte seqüência exata em $B$ mod:

$$
0 \longrightarrow F\left(X\left(i^{\prime}\right)\right) \longrightarrow P^{\prime}(i) \stackrel{F\left(\beta\left(i^{\prime}\right)\right)}{\longrightarrow} \triangle_{i}^{\prime} \longrightarrow 0 .
$$

Seja $j>i$ e consideremos $g \in \operatorname{Hom}_{A}\left(P^{\prime}(j), P^{\prime}(i)\right)=\operatorname{Hom}_{A}\left(F\left(T\left(j^{\prime}\right), F\left(T\left(i^{\prime}\right)\right)\right.\right.$, onde $j^{\prime}=n-j+1<i^{\prime}$. Como o funtor $F$ é uma equivalência contravariante entre $\mathcal{F}(\triangle)$ e $F(\mathcal{F}(\triangle)) \subset B \bmod (\operatorname{Lema3.5.16}(2))$, temos que $g=F(h)$, para algum $h \in \operatorname{Hom}_{A}\left(T\left(i^{\prime}\right), T\left(j^{\prime}\right)\right)$. Da mesma forma como o observado para o índice $i^{\prime}$, a Proposição 3.5.13 garante que s fatores de composição de $T\left(j^{\prime}\right)$ são simples $S_{k}$, com $k \leq j^{\prime} \leq i^{\prime}$. Assim, pelo Lema 3.2.7, obtemos que $\operatorname{Hom}_{A}\left(\triangle_{i^{\prime}}, T\left(j^{\prime}\right)\right)=0$ e, portanto, a composta $h \beta\left(i^{\prime}\right)=0$. Em consequência temos que

$$
\operatorname{Hom}_{A}\left(h \beta\left(i^{\prime}\right), T\right)=\operatorname{Hom}_{A}\left(\beta\left(i^{\prime}\right), T\right) \operatorname{Hom}_{A}(h, T)=\operatorname{Hom}_{A}\left(\beta\left(i^{\prime}\right), T\right) g=0,
$$

e, portanto, que $\operatorname{Im} g \subseteq F\left(X\left(i^{\prime}\right)\right)$. A seqüência exata (3.8) e este ultimo fato, mostraram que $\triangle_{i}^{\prime}$ é o máximo quociente de $P^{\prime}(i)$ com fatores de composição com índice no máximo $i$. Portanto esta provado que $\triangle^{\prime}=\left(\triangle_{1}^{\prime}, \triangle_{2}^{\prime}, \ldots, \triangle_{n}^{\prime}\right)$, onde $\triangle_{i}^{\prime}=\operatorname{Hom}_{A}\left(\triangle_{n-i+1}, T\right)$ é a seqüência de $B$-módulos (à esquerda) estandares.

Mostremos por fim que ${ }_{B} B \in \mathcal{F}\left(\triangle^{\prime}\right)$. Como para cada $i=1,2, \ldots, n$, $P^{\prime}(i)=F(T(n-i+1))$ e $T(n-i+1) \in \mathcal{F}(\triangle)$ temos, pelo Lema 3.5.16 (3), que $P^{\prime}(i)$ tem uma $\triangle^{\prime}$-filtração, ou seja $P^{\prime}(i) \in \mathcal{F}\left(\triangle^{\prime}\right)$. $\operatorname{Logo}_{B} B \in \mathcal{F}\left(\triangle^{\prime}\right)$ e esta provado que $B$ é estandarmente estratificada, com relação à sequiência $\triangle^{\prime}$ de $B$-módulos à esquerda.

A Proposição 3.5.17 garante que a álgebra $B=\operatorname{End}_{A}(T)$ é estandarmente 
Álgebras estandarmente estratificadas e módulos inclinantes

estratificada (à esquerda) e, por seu lado, o Teorema 3.5.5 garante que existe um $B$-módulo (à esquerda) inclinante característico ${ }_{B} T^{\prime}$, então surge a pergunta: a álgebra dos endomorfismos de $T^{\prime}, A^{\prime}=\operatorname{End}_{A}\left(T^{\prime}\right)$, é equivalente à álgebra original $A$ ? antes para responder esta questão, necessitamos do lema que segue, que a exemplo do Lema 3.5.16, relaciona também a categoria $\mathcal{F}(\triangle)$ e a sua imagem $F(\mathcal{F}(\triangle))$ em $B$ mod, onde $F$ é o funtor $\operatorname{Hom}_{A}(\ldots, T)$.

Lema 3.5.18. Sejam A uma álgebra estandarmente estratificada, $T$ o módulo inclinante característico associado e $B=\operatorname{End}_{A}(T)$. Se $X, Y \in \mathcal{F}(\triangle)$, então

$$
\operatorname{Ext}_{B}^{1}(F(Y), F(X)) \cong \operatorname{Ext}_{A}^{1}(X, Y),
$$

onde $F\left(\_\right)=\operatorname{Hom}_{A}\left(\_, T\right)$.

Demonstração. Suponhamos que $X, Y \in \mathcal{F}(\triangle)$. Então, pela Observação 3.5.2 (2), existe uma seqüência exata da forma $0 \longrightarrow Y \longrightarrow T_{0} \longrightarrow Y_{0} \longrightarrow 0$, com $T_{0} \in \mathcal{F}(\triangle) \cap \mathcal{Y}(\triangle)=\operatorname{add} T$ e $Y_{0} \in \mathcal{F}(\triangle)$. Aplicando o funtor $\operatorname{Hom}_{A}(\ldots, T)$, que é exato em $\mathcal{F}(\triangle)$, obtemos a sequiência exata

$$
0 \longrightarrow F\left(Y_{0}\right) \longrightarrow F\left(T_{0}\right) \longrightarrow F(Y) \longrightarrow 0 .
$$

Agora aplicando o funtor $\operatorname{Hom}_{B}(\ldots, F(X))$ na anterior seqüência obtemos a seqüência exata longa

$\cdots \longrightarrow \operatorname{Hom}_{B}\left(F\left(T_{0}\right), F(X)\right) \longrightarrow \operatorname{Hom}_{B}\left(F\left(Y_{0}\right), F(X)\right) \longrightarrow \operatorname{Ext}_{B}^{1}(F(Y), F(X))$

$\longrightarrow \operatorname{Ext}_{B}^{1}\left(F\left(T_{0}\right), F(X)\right) \longrightarrow \cdots$.

Notemos que $\operatorname{Ext}_{B}^{1}\left(F\left(T_{0}\right), F(X)\right)=0$, pois $F\left(T_{0}\right)$ é um $B$-módulo proje- 
Álgebras estandarmente estratificadas e módulos inclinantes

tivo. Usando o Lema 3.5.16 (2) obtemos o seguinte diagrama comutativo

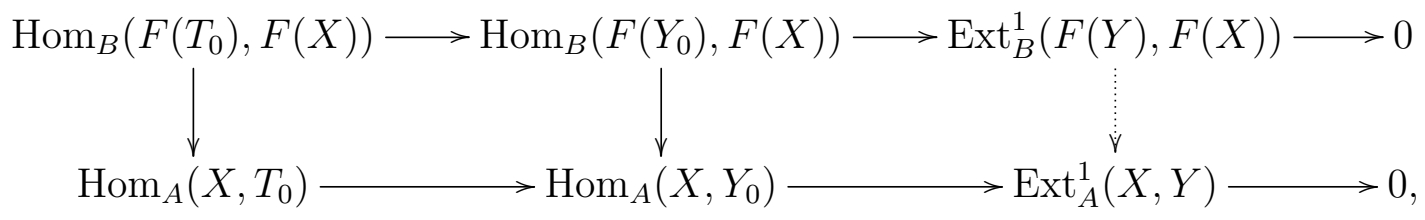

onde os dois primeiros homomorfismos verticais são isomorfismos. Pela passagem aos conúcleos, obtemos que $\operatorname{Ext}_{B}^{1}(F(Y), F(X)) \cong \operatorname{Ext}_{A}^{1}(X, Y)$.

Assim a resposta à questão colocada anteriormente sobre a relação entre as álgebras $\operatorname{End}_{B}\left(T^{\prime}\right)$ e a álgebra $A$ estandarmente estratificada original é dada pelo seguinte teorema.

Teorema 3.5.19. Sejam A uma $K$-álgebra estandarmente estratificada, $T$ o A-módulo (à diereita) inclinante característico associado. Seja $T^{\prime}$ o $B$ módulo (à esquerda) inclinante característico associado à álgebra estandarmente estratificada $B=\operatorname{End}_{A}(T)$. Então a $K$-álgebra $\operatorname{End}_{B}\left(T^{\prime}\right)$ é Morita equivalente a $A$ (isto é $\bmod A^{\prime}$ é equivalente a $\bmod A$ ).

Demonstração. Como $A_{A}$ é um progerador basta provar que

$$
A^{\prime}=\operatorname{End}_{B}\left(T^{\prime}\right) \cong \operatorname{End}_{A}(A) \cong A .
$$

Como $A$ é estandarmente estratificada, pela Proposição 3.4.2, $P_{i} \in \mathcal{F}(\triangle)$, para $1 \leq i \leq n$, e portanto, pelo Lema 3.5.16 (3) o $\operatorname{Hom}_{A}\left(P_{i}, T\right)=F\left(P_{i}\right) \in$ $\mathcal{F}\left(\triangle^{\prime}\right)$. De outro lado, pelo Lema 3.5.18, temos que $\operatorname{Ext}_{B}^{1}\left(F(Y), F\left(P_{i}\right)\right) \cong$ $\operatorname{Ext}_{A}^{1}\left(P_{i}, Y\right)=0$, para todo $Y \in \mathcal{F}(\triangle)$, isto é, $F\left(P_{i}\right) \in \mathcal{F}\left(\triangle^{\prime}\right) \cap \mathcal{Y}\left(\triangle^{\prime}\right)=$ add $T^{\prime}$. Desde que $P_{i}$ é um $A$-módulo indecomponível, o $B$-módulo $F\left(P_{i}\right)$ é também indecomponível; mais ainda, $\operatorname{Hom}_{A}\left(P_{i}, T\right) \supsetneqq \operatorname{Hom}_{A}\left(P_{j}, T\right)$, se $i \neq$ 
j. Como, pela Observação 3.5.4, $T^{\prime}$ tem $n$ somandos indecomponíveis não isomorfos, concluímos que $\oplus_{i=1}^{n} F\left(P_{i}\right) \cong T^{\prime}$. Assim temos que

$$
A \cong \operatorname{End}_{A}(A) \cong \operatorname{End}_{A}\left(\bigoplus_{i=1}^{n} P_{i}\right) \cong \operatorname{End}_{B}\left(F\left(P_{i}\right)\right) \cong \operatorname{End}_{B}\left(T^{\prime}\right)
$$

Portanto $\operatorname{End}_{B}\left(T^{\prime}\right) \cong \operatorname{End}_{A}(A) \cong A$, como queríamos.

\section{6 Álgebras quase-hereditárias}

Nesta seção abordaremos duas caracterizações diferentes das álgebras quasehereditárias. A primeria corresponde a definição original de tais álgebras dada por Cline, Parshall e Scott em termos de ideais hereditários e cadeias de hereditariedade. Mostraremos que tal definição é equivalente a dada na Seção 3.4. A segunda caracterização diz que as álgebras quase-hereditárias não são outras que as álgebras estandarmente estratificadas de dimensão global finita. Cabe resaltar que embora demonstrações destas caracterizações existam na literatura as aqui apresentadas são originais.

Começamos definindo os conceitos de ideal hereditário e de cadeia de hereditariedade.

Definição 3.6.1. Sejam A um anel e $J$ um ideal bilateral e não nulo de A. $O$ ideal $J$ chama-se hereditário se $J^{2}=J$, JradAJ $=0$, e $J$ considerado como A módulo à direita é projetivo.

Uma cadeia do tipo $0=J_{0} \subseteq J_{1} \subseteq \ldots \subseteq J_{t-1} \subseteq J_{t} \subseteq \ldots \subseteq J_{m}=A$ de ideais de $A$ tais que para $1 \leq t \leq m, J_{t} / J_{t-1}$ é um ideal hereditário de $A / J_{t-1}$ é chamada de cadeia de hereditariedade de $A$. 
Todas as álgebras semisimples tem uma cadeia de hereditariedade, basta tomar a cadeia de ideais $0 \subseteq A \subseteq A$, o quociente $A / 0 \cong A$ é um ideal de hereditariedade de $A / 0 \cong A$ pois $A_{A}$ é projetivo, $A^{2}=A$ e $A(\operatorname{rad} A) A=0$, pois $\operatorname{rad} A=0$.

Para caracterizar as álgebras quase-hereditárias em termos das cadeias de hereditariedade usaremos o seguinte lema, cuja demonstração pode ser encontrada em [8].

Lema 3.6.2. Se A é uma álgebra com cadeia de hereditariedade, então os idempotentes primitivos podem ser reordenados de tal forma que

$$
0 \subset A e_{1} A \subset A\left(e_{1}+e_{2}\right) A \subset \ldots \subset A\left(e_{1}+e_{2}+\ldots+e_{n}\right) A=A
$$

é uma cadeia de hereditariedade para A. Além disso, a ordem definida no lema anterior define uma ordem na qual ela é quase-hereditária.

Proposição 3.6.3. A álgebra A é quase-hereditária se, e somente se, tem uma cadeia de hereditariedade.

Demonstração. Suponhamos que $A$ é uma álgebra quase-hereditária na ordem $e=\left(e_{1}, e_{2}, \ldots, e_{n}\right)$. Usando a notação adotada na Seção 3.3, demonstraremos que a filtração traço

$$
0=A^{(n+1)} \subset A^{(n)} \subset \cdots \subset A^{(2)} \subset A^{(1)}=A
$$

é uma cadeia de hereditariedade para $A$, onde $A^{(i)}=\tau_{\varepsilon_{i} A}(A)=A \varepsilon_{i} A$. Em primeriro lugar é claro que $A^{(i)}$ é um ideal bilateral e idempotente $A$. Agora, como $A$ é estandarmente estratificada, então pelo Corolário 3.3.4 temos que 
$A^{(i)} / A^{(i+1)}$ é um $A / A^{(i+1)}$-módulo projetivo, para $i=1, \ldots, n$. Para finalizar falta verificar que $A^{(i)} / A^{(i+1)}\left(\operatorname{rad} A / A^{(i+1)}\right) A^{(i)} / A^{(i+1)}=0$, para tal notemos que

$$
\begin{aligned}
A^{(i)} / A^{(i+1)}\left(\operatorname{rad} A / A^{(i+1)}\right) A^{(i)} / A^{(i+1)} & \cong A e_{i} A^{\operatorname{rad} A+A^{(i+1)}} A e_{i} A \\
& \cong \frac{A e_{i} A(\operatorname{rad} A) A e_{i} A+A e_{i} A\left(A^{(i+1)}\right) A e_{i} A}{A^{(i+1)}} .
\end{aligned}
$$

Como $A$ é quase-hereditária, em virtude da Proposição 3.4.7, temos que

$$
\begin{aligned}
A e_{i} A(\operatorname{rad} A) A e_{i} A & =A e_{i}(\operatorname{rad} A) e_{i} A \\
& =A e_{i} A \varepsilon_{i+1} A e_{i} A \\
& =A e_{i} A\left(A^{(i+1)}\right) A e_{i} A .
\end{aligned}
$$

Mas como $A^{(i+1)}$ é um ideal bilateral de $A$, então $A e_{i} A\left(A^{(i+1)}\right) A e_{i} A \subset A^{(i+1)}$, portanto $A^{(i)} / A^{(i+1)}\left(\operatorname{rad} A / A^{(i+1)}\right) A^{(i)} / A^{(i+1)}=0$ e em comseqüênicia (3.9) é uma cadeia de hereditariedade para $A$.

Reciprocamente, suponhamos que $A$ tem uma cadeia de hereditariedade, pelo Lema 3.6.2 existe uma cadeia de hereditariedade de $A$ da forma

$$
0 \subset A e_{1} A \subset A\left(e_{1}+e_{2}\right) A \subset \ldots \subset A\left(e_{1}+e_{2}+\ldots+e_{n}\right) A=A .
$$

Afirmamos que a $K$-álgebra $A$ é estandarmente estrafiticada na ordem $\mathbf{e}=$ $\left(e_{n}, e_{n-1}, \cdots, e_{2}, e_{1}\right)$. De fato, desde que

$$
A\left(e_{1}+\ldots+e_{i}+e_{i+1}\right) A / A\left(e_{1}+\ldots+e_{i}\right) A=A^{(i+1)} / A^{(i)},
$$

é um $A / A^{(i)}$-módulo projetivo, para $i=1, \ldots, n$, então, em vitude do Corolário 3.3.4, $A \in \mathcal{F}(\triangle)$, isto é $A$ é uma álgebra estandarmente estratificada na ordem e. 
Nosso intuito agora é mostrar que as álgebras estandarmente estratificadas de dimensão global finita são quase-hereditárias, para tal introduzimos o seguinte conceito.

Definição 3.6.4. Sejam A uma K-álgebra e $\triangle$ o conjunto de módulos estandares relativos a ordem e de um conjunto completo de idempotentes primitivos e ortogonais de $A$. Denotamos por $K_{0}(\triangle)$ o grupo abeliano $K_{0}(\triangle)=$ $F_{\triangle} / F_{\triangle}^{\prime}$, onde $F_{\triangle}$ é o grupo abeliano livre cuja base é o conjunto de classes de isomorfismo $\widetilde{M}$ de módulos $M$ em $\mathcal{F}(\triangle)$ e $F_{\triangle}^{\prime}$ é o subgrupo de $F_{\triangle}$ gerado pelos elementos da forma $\widetilde{M}-\widetilde{L}-\widetilde{N}$ correspondentes as seqüências exatas curtas $0 \rightarrow L \rightarrow M \rightarrow N \rightarrow 0$ em $\mathcal{F}(\triangle)$.

Denotamos por $[M]$ a imagem de $\widetilde{M}$ pelo epimorfismo canônico $\pi$ : $F_{\triangle} \longrightarrow F_{\triangle} / F_{\triangle}^{\prime}$. Definimos a função $\operatorname{dim}_{\triangle}: K_{0}(\triangle) \longrightarrow \mathbb{Z}^{n}$, por $\operatorname{dim}_{\triangle}([M])=$ $\left(\left[M: \triangle_{1}\right], \ldots,\left[M: \triangle_{n}\right]\right)^{t}$. Desde que $\left[M: \triangle_{i}\right]$, para $i=1, \ldots, n$, não depende da $\triangle$-filtração de $M$, a função $\operatorname{dim}_{\triangle}$ esta bem definida. Mais ainda, $\operatorname{dim}_{\triangle}$ é um isomorfismo de grupos como veremos na seguinte proposição, cuja demonstração é análoga à do Teorema 3.5, Cap. III de [4].

Proposição 3.6.5. $K_{0}(\triangle)$ é um grupo abeliano livre com base $\left\{\left[\triangle_{i}\right]\right\}_{i=1}^{n}$ e a função $\operatorname{dim}_{\triangle}$ é um isomorfismo de grupos.

Observação 3.6.6. Desde que, pela Proposição 3.1 .4 o homomorfismo de grupos $\underline{\operatorname{dim}}: K_{0}(A) \longrightarrow \mathbb{Z}^{n}$, definido por $\underline{\operatorname{dim}}([M])=\left(\left[M: S_{1}\right], \ldots,\left[M: S_{n}\right]\right)^{t}$ é um isomorfismo, pode-se considerar a composta

$$
(\underline{\operatorname{dim}})^{-1} \operatorname{dim}_{\triangle}: K_{0}(\triangle) \longrightarrow K_{0}(A),
$$


Se escrevemos a matriz de tal homomorfismo nas bases $\left\{\left[\triangle_{i}\right]\right\}_{i=1}^{n}$ e $\left\{\left[S_{i}\right]\right\}_{i=1}^{n}$ para $K_{0}(\triangle)$ e $K_{0}(A)$, respectivamente, obtemos

$$
D=\left[\begin{array}{cccc}
{\left[\triangle_{1}: S_{1}\right]} & {\left[\triangle_{2}: S_{1}\right]} & \ldots & {\left[\triangle_{n}: S_{1}\right]} \\
{\left[\triangle_{1}: S_{2}\right]} & {\left[\triangle_{2}: S_{2}\right]} & \ldots & {\left[\triangle_{n}: S_{2}\right]} \\
{\left[\triangle_{1}: S_{3}\right]} & {\left[\triangle_{2}: S_{3}\right]} & \ldots & {\left[\triangle_{n}: S_{3}\right]} \\
\vdots & \vdots & & \vdots \\
{\left[\triangle_{1}: S_{n}\right]} & {\left[\triangle_{2}: S_{n}\right]} & \ldots & {\left[\triangle_{n}: S_{n}\right]}
\end{array}\right] .
$$

Como os factores de composição do módulo $\triangle_{i}$ são módulos simples $S_{j}$, com $j \leq i$, então podemos escrever

$$
D=\left[\begin{array}{cccc}
{\left[\triangle_{1}: S_{1}\right]} & {\left[\triangle_{2}: S_{1}\right]} & \ldots & {\left[\triangle_{n}: S_{1}\right]} \\
0 & {\left[\triangle_{2}: S_{2}\right]} & \ldots & {\left[\triangle_{n}: S_{2}\right]} \\
0 & 0 & \ldots & {\left[\triangle_{n}: S_{3}\right]} \\
\vdots & \vdots & & \vdots \\
0 & 0 & \ldots & {\left[\triangle_{n}: S_{n}\right]}
\end{array}\right]
$$

Portanto temos que $\operatorname{det} D=\left[\triangle_{1}: S_{1}\right]\left[\triangle_{2}: S_{2}\right] \cdots\left[\triangle_{n}: S_{n}\right]>0$.

Corolário 3.6.7. Se A é uma K-algebra estandarmente estratificada, então A é quase-hereditária se, e somente se, $\operatorname{det} D=1$.

Demonstração. Decorre imediatamente da anterior observação e da definiçâo de álgebra quase-hereditária.

Corolário 3.6.8. Se A é uma K-álgebra estandarmente estratificada, então o determinante da matriz de Cartan de A é postivo.

Demonstração. Se $A$ é uma álgebra estandamente estratificada, então pelo Corolário 3.4.14 todos os módulos em $\mathcal{F}(\triangle)$ tem dimensão projetiva finita. Isto significa que o conjunto $\left\{\left[P_{i}\right]\right\}_{i=1}^{n}$ é tambem uma base do grupo 
$K_{0}(\triangle)$. Escrevendo a matriz de $(\underline{\operatorname{dim}})^{-1} \operatorname{dim}_{\triangle}$ referente as bases $\left\{\left[P_{i}\right]\right\}_{i=1}^{n}$ e $\left\{\left[S_{i}\right]\right\}_{i=1}^{n}$ para $K_{0}(\triangle)$ e $K_{0}(A)$, respectivamente, obtemos

$$
C=\left[\begin{array}{cccc}
{\left[P_{1}: S_{1}\right]} & {\left[P_{2}: S_{1}\right]} & \ldots & {\left[P_{n}: S_{1}\right]} \\
{\left[P_{1}: S_{2}\right]} & {\left[P_{2}: S_{2}\right]} & \ldots & {\left[P_{n}: S_{2}\right]} \\
{\left[P_{1}: S_{3}\right]} & {\left[P_{2}: S_{3}\right]} & \ldots & {\left[P_{n}: S_{3}\right]} \\
\vdots & \vdots & & \vdots \\
{\left[P_{1}: S_{n}\right]} & {\left[P_{2}: S_{n}\right]} & \ldots & {\left[P_{n}: S_{n}\right]}
\end{array}\right]
$$

Note-se que $C$ é matriz de Cartan de $A$ e que se $D$ é a matriz da Observação 3.6.6 então $C$ e $D$ são matrizes semelhantes. Portanto, de acordo com o Corolário 3.6.7, temos que o determinante de $C=C_{A}$ é positivo.

Proposição 3.6.9. Seja A uma K-álgebra estandarmente estratificada com dimensão global finita. Então A é quase-hereditária.

Demonstração. Pela Proposição 3.10, do Cap III, em [4], temos que se gld $A<\infty$, então $\operatorname{det} C_{A} \in\{-1,1\}$. Mas $\operatorname{det} A>0$, pelo Corolário 3.6.8, então det $C_{A}=1$. Portanto, pelo Corolário 3.6.7, $A$ é quase-hereditária.

Como consequencia esta ultima proposição e do Corolário 3.4.13, temos a seguinte caracterização das álgebras quase-hereditárias.

Teorema 3.6.10. Seja A uma K-álgebra estandarmente estratificada. Então $A$ é quase-hereditária se, e somente se, gld $A<\infty$. 


\section{Referências Bibliográficas}

[1] I. Ágoston, D. Happel, E. Lukács and L. Unger. Finitistic dimension of standardly stratified algebras. Comm. Algebra 28 (2000), 1745-2752.

[2] I. Ágoston, D. Happel, E. Lukács and L. Unger. Standardly Stratified Algebras and Tilting. Journal of Algebra 226, 144-160 (2000).

[3] Assem, I. Algèbres et modules, série enseignement des mathématiques, Presses de l'Université d'Ottawa (Ottawa). Masson (Paris), 1977.

[4] I. Assem, D. Simson and A. Skowronski. Elements of the Representation Theory of Associative Álgebras. 1: Techniques of Representation Theory. Series: London Mathematical Society Student Texts 65. Cambridge University Press, 2006.

[5] M. Auslander, and I. Reiten. Applications of contravariantly finite subcategories. Advances in mathematics 86,111-152 (1991).

[6] M. Auslander, I. Rieten and S. Smalø. Representation Theory of Artin Algebras. Cambridge Studies in Avanced Mathemathics 36. Cambridge Academic University Press, 1995.

[7] M. Auslander and S. Smalø. Almost split sequences in subcategories. J. Algebra 69 (1981), $426-454$. 
[8] W. D. Burgess and K. R. Fuller. On quasihereditary rings. Proceedings of the Americal Mathematical Society 106 (1989), no. 2, 321-328.

[9] E. Cline, B. Parshall and L. Scott. Finite dimensional and highest weight categories. J. Reine Angew. Math, 391 (1988), 85-99.

[10] E. Cline, B. Parshall and L. Scott. Stratifying endomorphism algebras. Mem Amer. Soc. 124 (1996), no.591.

[11] E. Cline, B. Parshall and L. Scott. Derived categories, quasi hereditary algebras, and algebraic groups. Proc. Ottawa-Moosonne Workshop, Carleton-Ottawa Math. Lecture Note Series 3 (1988), 1-105.

[12] I. V. Dlab, and C. M. Ringel. The module theoretical appoach to quasihereditary algebras. London Math. Soc. Lecture note series 168. Cambridge Univ. Press (1992), 200-224.

[13] I. V. Dlab, C. M. Ringel. Quasi-hereditary algebras. Illinois J. Math. 33 (1989), 280-291.

[14] I. V. Dlab, Properly stratified algebras. C. R. Acad. Sci. Paris, Série 331 (2000),191-196.

[15] Yu. A. Drozd and V. V. Kirichenkco. Finite dimensional algebras. Apendix on quasi- hereditary algebras por V. Dlab. Springer-Verlang, Berlin, 1994.

[16] K. Erdmann and C. Sáenz. On Standarly Stratified Algebras. Comm. Algebra 31 (2003), no.7, 3429-3446.

[17] D. Happel. Triangulated categories in representation theory of finite dimensional algebras. London Mathematical Society Lecture Note Series 119. Cambridge University Press (1988). 
[18] F. Hernández and E. Marcos. Algebras.Standarly Stratified in all orders. Colloquium Mathematicum. 107 (2007),81-87.

[19] F. Anderson and K.Fuller. Rings and categories of modules. Graduate Texts in Mathematics. Springer-Verlang, 1992.

[20] E. Marcos, O. Mendoza and C. Sáenz. Stratifying systems via relative projective modules. Comm. Algebra 33 (2005), no.5, 1559- 1573.

[21] E. Marcos, O. Mendoza and C. Sáenz. Stratifying systems via relative simple modules. J. Algebra 280 (2004), no.2, 472-487.

[22] E. Marcos, O. Mendoza and C. Sáenz. Applications of stratifying systems to the finitistic dimension. J. Pure App. Algebra 205 (2006), no. 2, 393-411.

[23] Y. Miyashita. Tilting modules of finite projective dimension. Math. Z. 193, (1996), 113-146.

[24] V. M. Ringel. The category of good modules over a quasi-hereditary algebras . Proceedings of the Sixth International Conference on Representations of Algebras (Ottawa, ON, 1992), 17 pp., Carleton-Ottawa Math. Lecture Note Ser., 14, Carleton Univ., Ottawa, ON, 1992.

[25] V. M. Ringel. The category of modules with good filtrations over a quasihereditary algebra has almost split sequences. Math. Z. 208 (1991), no. 2, 209-223.

[26] C. Xi. Standardly stratified algebras and cellular algebras. Math. Proc. Cambridge Philos. Soc. 133 (2002), no 1, 37-53. 


\section{Índice Remissivo}

$\theta$-filtração, 14

álgebra básica, 2

álgebra coestandarmente estratificada, 47

álgebra conexa, 2

álgebra de caminhos, 4

álgebra estandarmente

estratificada, 47

álgebra indecomponível, 2

álgebra quase-hereditária, 51

álgebras quase-hereditárias, v, 29

apresentação, 6

aproximação à direita, 16

aproximação à esquerda, 17

caminho, 4

carcás, 3

carcás com relações, 5

carcás conexo, 4

carcás de Auslander-Reiten, 13

carcás ordinário, 6

categoria contravariantemente finita, 17

categoria corresolvente, 49

categoria covariantemente finita, 17

categoria fechada por extensões, 15

categoria fechada por somandos

diretos, 15

categoria funtorialmente finita, 17

categoria resolvente, 49

começo, 4

dimensão global, 3

dimensão projetiva, 3

dimensão projetiva finitistica, 67

epimorfismo que cinde, 10

final, 4

flechas, 3

grafo subjacente, 4

grupo de Grothendieck, 31

homomorfismo irredutível, 11

homomorfismo minimal, 10, 11 
homomorfismo quase-cindido, 11

ideal admissível, 5

módulo coinclinante

generalizado, 61

módulo Ext-injetivo, 25

módulo Ext-projetivo, 25

módulo inclinante generalizado, 60

módulo relativamente injetivo, 25

módulo relativamente projetivo, 25

módulo Schurian, 49, 50

módulos coestandares, 35

módulos estandares, 35

matriz de Cartan, 32

monomorfismo que cinde, 10

relação, 5

representação do carcás, 7

resolução projetiva, 2

retração, 10

seção, 10

seqüência quase-cindida, 12

seqüência Schurian, 49

seqüências de Auslander-Reiten, 12

relativas, 27,42

suporte, 44

Teorema de Auslander-Reiten, 12
Teorema de Gabriel, 6

traço, 33

vértices, 3

vetor dimensão, 31 